\title{
Fibrations and globalizations of compact homogeneous CR-manifolds*
}

\author{
B. Gilligan and A. Huckleberry \\ July 31, 2021 \\ In memory of A.G. Vitushkin
}

\section{Contents}

1 Introduction 3

2 Basics on CR-manifolds 5

2.1 CR-Structures . . . . . . . . . . . . . . 5

2.2 The Levi Form . . . . . . . . . . . . . . . . . . . . . . . 9

2.3 Homogeneous CR-structures . . . . . . . . . . . . . . . . . . . . . . . . . . . . . . .

2.4 Complexification of the Lie algebra . . . . . . . . . . 10

2.5 The $G$-action on $\widehat{\mathfrak{g}} \ldots \ldots \ldots$. . . . . . . . . . . . . 11

2.6 The $\mathfrak{g}$-anticanonical fibration . . . . . . . . . . . . . . . . . . . . . . . . 12

2.6.1 Equivariant CR-Fibrations . . . . . . . . . . . 12

2.6.2 Another Construction and Further Properties . . . . . 15

2.7 Globalization of the Fiber . . . . . . . . . . . . . 18

3 Globalization 19

3.1 Homogeneous fibrations . . . . . . . . . . . . . . 20

3.2 Remarks on coverings . . . . . . . . . . . . . . . . 23

3.3 The case of the $\mathfrak{g}$-anticanonical fibration . . . . . . . . 25

*This project was partially supported by an NSERC Discovery Grant and by SFB/TR 12 of the Deutsche Forschungsgemeinschaft. 
5 Projective homogeneous spaces of codimension at most two 30 5.1 Description of the projective globalization . . . . . . . 30

5.1 .1 The spherical property . . . . . . . . . . . . 30

5.1 .2 Affine-Rational fibrations . . . . . . . . . . . . . 32

5.1.3 The fiber of an affine-rational fibration . . . . . . . . 33

5.2 The case of a nontrivial radical . . . . . . . . . . 35

5.3 Transitive action on the base . . . . . . . . . . . 36

5.3.1 Transitive action of a simple real form . . . . . . . 37

5.3.2 Simple real forms which are complex . . . . . . . . . 40

5.3.3 The case of several factors . . . . . . . . . . . . 42

5.4 Actions of real forms on flag manifolds . . . . . . . . . . 44

5.4 Background . . . . . . . . . . . . . . . 45

5.4.2 Closed orbits of codimension one . . . . . . . . . 46

5.4 .3 Closed orbits of codimension two . . . . . . . . . 50

5.5 Classification theorem . . . . . . . . . . . . 54

5.6 Application to globalization . . . . . . . . . . . . 60

5.6 .1 On the role of the affine quadric . . . . . . . . . 60

5.6 .2 On the role of real projective space . . . . . . . . 64

5.6 .3 Conditions for globalization . . . . . . . . . . . 64

6 Fine Classification 66

6.1 The Levi-nondegenerate Case . . . . . . . . . . . . . . . 66

6.2 The Kählerian Parallelizable Case . . . . . . . . . . . . . 72

\begin{abstract}
Fibrations methods which were previously used for complex homogeneous spaces and CR-homogenous spaces of special types $([\mathrm{HO}]$, [AHR], [HR, Ri]) are developed in a general framework. These include the $\mathfrak{g}$-anticanonical fibration in the CR-setting which reduces certain considerations to the compact projective algebraic case where a Borel-Remmert type splitting theorem is proved. This allows a reduction to spaces homogeneous under actions of compact Lie groups. General globalization theorems are proved which allow one to regard the homogeneous $\mathrm{CR}$-manifold as the orbit of a real Lie group in a complex homogeneous space of a complex Lie group. In the special case of $\mathrm{CR}$-codimension at most two precise classification results are
\end{abstract}


proved and are applied to show that in most cases there exists such a globalization.

\section{Introduction}

In this paper we consider homogeneous $\mathrm{CR}$-manifolds ( $\mathrm{CR}=$ Cauchy-Riemann). They are assumed to be of the form $M=G / H$ with $G$ being a connected Lie group acting on $M$ by CR-automorphisms. We present a number of Lie theoretic methods involving equivariant CR-fibrations and globalizations for studying these manifolds. These are applied to prove structure and classification results in projective and low-codimensional settings. It should be emphasized that, although we only consider the setting where a Lie group is acting transitively on our CR-manifold, its full group of CR-automorphisms could be infinite-dimensional.

An equivariant CR-fibration is just a CR-mapping $G / H \rightarrow G / I$ of homogeneous CR-manifolds. In our context the notion of globalization is defined as follows. Since $M$ is real-analytic, it is embedded (uniquely at the level of germs) as a generic submanifold of a complex manifold $X$. If $X$ is chosen sufficiently small, then the Lie algebra $\widehat{\mathfrak{g}}:=\mathfrak{g}+i \mathfrak{g}$ acts locally and locally transitively on $X$. We say that the $G$-action on $M$ can be globalized if there is a connected complex Lie group $\widehat{G}$ with Lie algebra $\widehat{\mathfrak{g}}$ such that $X$ can be taken to be a global complex homogeneous manifold $\widehat{G} / \widehat{H}$, i.e., $M$ is just a $G$-orbit in such a manifold.

If $\widehat{G}$ is a complex Lie group and $\widehat{H}$ is a closed complex subgroup of $\widehat{G}$, then one lets $\widehat{N}:=N_{\widehat{G}}\left(\widehat{H}^{\circ}\right)$ be the normalizer in $\widehat{G}$ of the connected component of the identity of $\widehat{H}$ and then obtains an important tool, the normalizer fibration $\widehat{G} / \widehat{H} \rightarrow \widehat{G} / \widehat{N}$. This has proved very fruitful, since the analytic problems caused by the discrete isotropy of its fiber and the methods of algebraic groups that can be applied to its base (this is equivariantly embedded into some projective space) are now separated and can be analyzed somewhat independently.

In the case of a homogeneous CR-manifold $M=G / H$ an analogue of the normalizer fibration, the $\mathfrak{g}$-anticanonical fibration, is known to exist. One may attempt to use this fibration,

$$
G / H \stackrel{F}{\rightarrow} G / J
$$


to build a globalization of the given $M$. The base of this fibration is globalizable, because it is a $G$-orbit in a projective space. Thus one must determine whether or not the parallelizable fiber $F=J / H$ is globalizable and, if so, if its globalization can be fit together with the globalization of the base.

In the second section of this paper we recall the necessary basics about CRmanifolds, the construction and properties of the $\mathfrak{g}$-anticanonical fibration, and show the existence of the globalization $\widehat{F}$ if $F$ has codimension at most two.

As indicated above, the base $G / J$ also has a globalization $\widehat{G} / \widehat{J}$ which is a $\widehat{G}$-orbit in projective space. The main point is to understand whether $\widehat{J}$ acts holomorphically on $\widehat{F}$. There is a surjectivity condition on the induced map of fundamental groups, which we call Condition (C), needed to deal with the possibility of ineffectivity of the $\widehat{G}$-action on the space $\widehat{G} / \widehat{J}$. If this condition is satisfied and if the radical $\widehat{R}$ of $\widehat{G}$ is acting as an Abelian group on the base $\widehat{G} / \widehat{J}$ (a necessary condition), then a globalization does exist. This result is proved in the general setting in Theorem 3.1 and in the particular setting of the $\mathfrak{g}$-anticanonical fibration in Theorem 3.2.

In the fourth section, using methods involving real and complex algebraic groups, we prove structure theorems for projectively embedded, compact, homogeneous CR-manifolds. We show that the complexified group $\widehat{G}$ is reductive, in particular that its radical $\widehat{R}$ is Abelian, and prove a splitting theorem which can be regarded as the analogue of the Borel-Remmert theorem for complex homogeneous spaces. The results in this setting are general and should prove useful for other problems.

In the fifth section, methods of algebraic groups are applied to give a detailed description of projective homogeneous $\mathrm{CR}$-manifolds of codimension at most two. In particular, the desired globalization results are obtained whenever the 2-dimensional affine quadric $\mathrm{SL}_{2}(\mathbb{C}) / \mathbb{C}^{*}$ is not involved.

A fine classification of compact homogeneous CR-hypersurfaces with nondegenerate Levi forms is known. In the last section we note that our classification results in section five give a classification of compact homogeneous CR-manifolds $M$ with nondegenerate Levi form in the codimension two setting. We also present remarks on the situation where $\widehat{G} / \widehat{H}$ is Kähler. 
This work is dedicated to the memory of Anatolii Georgievich Vitushkin with whom we had a continuous and extremely fruitful cooperation starting in the mid-1980's. Tolya was not only a mathematician of the highest quality but also a wonderful teacher whose students are working on a wide range of topics in the general area of complex analysis. Complex and CR-manifolds having a high degree of symmetry appear in many of their works. In our opinion a fusion of the methods which we present here and those developed by members of the Vitushkin School, which are complementary to ours, will lead to a deeper understanding of the role of symmetry in complex analysis.

Let us close this introduction by giving a brief guideline to the work in this direction of Vitushkin's students. A. Loboda (see, e.g., [L]) has proved classification results for locally homogeneous CR-manifolds in low-dimensions. V. Ezhov and V. Beloshapka and their coworkers (in particular G. Schmalz who was also in the Moscow seminar as a student of Shabat) have carried out computations of Chern-Moser invariants of CR-manifolds which in fact have a high degree of symmetry. The classification theorems of A. Isaev and N. Kruzhilin in situations where big unitary groups are acting (see [IK]) and the classification work of Isaev et al for domains and hyperbolic manifolds with large automorphism groups relative to the dimension of the domain (see [I] and its references) all involve settings where orbits of the real Lie group of interest are low-codimensional CR-manifolds which fit into the context of the present paper.

For other recent results in our context we refer to the work of G. Fels and W. Kaup (see e.g. [FK]). For classification results in the hypersurface case see, e.g., [MN], [N], [Ro], $[\mathrm{AHR}$, [Ri], [HR] and [AS].

Finally, we wish to thank the referee for his critical remarks. In our opinion they led to important improvements in this work.

\section{Basics on CR-manifolds}

\section{$2.1 \quad$ CR-Structures}

A CR-manifold is a real manifold that has some "partial complex structure". These manifolds have always been of interest, since they can occur in a number of natural ways. For example, any real-valued smooth function 
on a complex manifold defines a CR-submanifold at those points where its gradient does not vanish. Another important class of examples arises as the orbits of real subgroups in the homogeneous spaces of globalizations of those groups.

Definitions: Suppose $M$ is an $n$-dimensional (differentiable) manifold. A CR-structure of type $(n, l)$ on $M$ is a subbundle $H$ of rank $l$ of the complexified tangent bundle $T M \otimes \mathbb{C}$ that satisfies:

1) $H \cap \bar{H}=\{0\}$, the zero section of $T M \otimes \mathbb{C}$

2) $H$ is involutive

A CR-manifold of type $(n, l)$ is a pair $(M, H)$ consisting of an $n$-dimensional manifold $M$ and a CR-structure $H$ of type $(n, l)$ on $M$.

If $(M, H)$ is a CR-manifold of type $(n, l)$, then $H$ defines a subbundle $T^{C R} M$ of $T M$ of (real) rank $2 l$, called the holomorphic tangent bundle to $M$, that satisfies:

$\left.1^{\prime}\right)$ There is a bundle isomorphism $\mathcal{J}: T^{C R} M \rightarrow T^{C R} M$ such that $\mathcal{J}^{2}=$ $-\mathrm{Id}_{T^{C R} M}$

$\left.2^{\prime}\right)$ For all $\widehat{\xi}, \widehat{\zeta} \in \Gamma\left(M, T^{C R} M\right)$ one has $[\widehat{\xi}, \widehat{\zeta}]-[\mathcal{J} \widehat{\xi}, \mathcal{J} \widehat{\zeta}] \in \Gamma\left(M, T^{C R} M\right)$ and $N(\widehat{\xi}, \widehat{\zeta}):=\mathcal{J}([\widehat{\xi}, \widehat{\zeta}]-[\mathcal{J} \hat{\xi}, \mathcal{J} \widehat{\zeta}])-[\widehat{\xi}, \mathcal{J} \widehat{\zeta}]-[\mathcal{J} \widehat{\xi}, \widehat{\zeta}]=0$.

One has the equivalences 1) $\left.\Longleftrightarrow 1^{\prime}\right)$ and 2) $\left.\Longleftrightarrow 2^{\prime}\right)$. As well, there is the bundle map $\alpha: T^{C R} M \rightarrow H$ defined by $\alpha(\widehat{\xi}):=\frac{1}{2}(\widehat{\xi}-i \mathcal{J} \widehat{\xi})$ and $\alpha$ is an isomorphism that satisfies $\alpha(\mathcal{J} \widehat{\xi})=i \alpha(\widehat{\xi})$. Thus there is a one-to-one correspondence between CR-structures $H$ defined on $M$ and holomorphic tangent subbundles $T^{C R} M$ of $T M$.

CR-manifolds can arise as submanifolds embedded in complex manifolds. Suppose $(X, \widetilde{\mathcal{J}})$ is a complex manifold. Then $\widetilde{\mathcal{J}}$ can be uniquely extended to a complex linear endomorphism of the complexified tangent space $T X \otimes$ $\mathbb{C}$. Because $\widetilde{\mathcal{J}}^{2}=-\operatorname{Id}_{T X \otimes \mathbb{C}}$, the eigenvalues of $\widetilde{\mathcal{J}}$ are $+i$ and $-i$ and the corresponding eigenspaces of $T X \otimes \mathbb{C}$ are denoted, as is customary, by $T X^{1,0}$ and $T X^{0,1}$, respectively. Now assume $M \subset X$ is a (real) submanifold that satisfies the condition that

$$
H_{x}:=\left(T_{x} M \otimes \mathbb{C}\right) \cap T_{x} X^{1,0}
$$


has constant rank for all $x \in M$. Then $(M, H)$ is a CR-manifold. One should note that the CR-structure on $M$ is also given by

$$
\begin{aligned}
T^{C R} M & :=T M \cap \tilde{\mathcal{J}} T M \\
\mathcal{J} & :=\left.\widetilde{\mathcal{J}}\right|_{T^{C R} M}
\end{aligned}
$$

with $T_{x}^{C R} M$ having constant rank for all $x \in M$. Note that for $x \in M$ we have the following decomposition of the tangent space

$$
T_{x}(X)=T_{x}^{C R}(M) \oplus N_{x} \oplus \mathcal{J}\left(N_{x}\right),
$$

where

$$
T_{x}(M)=T_{x}^{C R}(M) \oplus N_{x},
$$

and $N$ is a subbundle of $T M$ complementary to $T^{C R}(M)$.

We say that $M$ has a generic CR-structure if $T M+\widetilde{\mathcal{J}} T M=\left.T X\right|_{M}$. (The sum is not direct, in general.)

Remark: If the CR-structure on $M$ has type $(n, l)$ and $M$ is embedded in a complex manifold $X$ as a generic submanifold, then the (real) codimension of this structure is given by

$$
\begin{aligned}
k & :=\operatorname{dim}_{\mathbb{R}} X-\operatorname{dim}_{\mathbb{R}} M \\
& =\operatorname{dim}_{\mathbb{R}} \mathcal{J}(N) \\
& =\operatorname{dim}_{\mathbb{R}} N \\
& =\operatorname{dim}_{\mathbb{R}} M-\operatorname{dim}_{\mathbb{R}} T^{C R} M \\
& =n-2 l ;
\end{aligned}
$$

this last formula also holds when $M$ is not embedded. It follows from this that

$$
\operatorname{dim}_{\mathbb{R}} X=n+k=2(n-l) ; \text { i.e., } \operatorname{dim}_{\mathbb{C}} X=n-l \text {. }
$$

Definition: Suppose $\left(M_{1}, T^{C R}\left(M_{1}\right), \mathcal{J}_{1}\right)$ and $\left(M_{2}, T^{C R}\left(M_{2}\right), \mathcal{J}_{2}\right)$ are two CRmanifolds. A smooth map $f: M_{1} \rightarrow M_{2}$ is called a CR-map if

$$
f_{*}\left(T_{x}^{C R}\left(M_{1}\right)\right) \subset T_{f(x)}^{C R}\left(M_{2}\right)
$$

for all $x \in M_{1}$ and

$$
\mathcal{J}_{2} \circ f_{*}=f_{*} \circ \mathcal{J}_{1}
$$


Concepts such as CR-submersion, CR-isomorphism, and CR-function are obvious.

Definition: A vector field $\widehat{\xi} \in \Gamma(M, T M)$ is called a CR-vector field if the local one-parameter group of transformation of $M$ that $\widehat{\xi}$ induces consists of CR-transformations. We will denote the set of CR-vector fields on $M$ by $\Gamma_{C R}(M, T M)$.

A CR-manifold $(M, H)$ is called an analytic CR-manifold if $M$ is an analytic manifold and $H$ is an analytic subbundle of $T M \otimes \mathbb{C}$; i.e., $H$ is locally generated by analytic local sections of $T M \otimes \mathbb{C}$.

Given a CR-manifold, one would be in an ideal situation if one could realize this manifold as an embedded manifold in a complex manifold from which it inherits its CR-structure.

An embedding $\sigma: M \rightarrow X$ of a CR-manifold into a complex manifold $X$ is called a CR-embedding if $\sigma(M) \subset X$ is a CR-submanifold and the map $\sigma$ is a CR-isomorphism of $M$ onto its image. Such an embedding is called generic if its image $\sigma(M)$ is a generic CR-submanifold of $X$. We will then say that $(X, \sigma)$ is a complexification of $M$.

Theorem 2.1 (Andreotti-Friedrichs $[\mathrm{AF}]$ ). Every analytic $C R$-manifold $M$ has a complexification $(X, \sigma)$ that is unique up to some biholomorphic map.

Theorem 2.2. $\quad$ 1. Suppose $f: M_{1} \rightarrow M_{2}$ is an analytic CR-map between two generic analytic $C R$-submanifolds $M_{i} \subset X_{i}, i=1,2$, where the $X_{i}$ are complex manifolds. Then there exist open neighborhoods $U_{i} \subset X_{i}$ of $M_{i}$ for $i=1,2$ and a holomorphic map $\widehat{f}: U_{1} \rightarrow U_{2}$ such that $\left.\widehat{f}\right|_{M_{1}}=f$.

2. A holomorphic map $\widehat{f}$ defined on an open, connected neighborhood of a generically embedded CR-manifold is constant if and only if $\widehat{f}_{M}$ is constant.

3. Suppose $M \subset X$ is a generic CR-submanifold of a complex manifold $X$ and $\widehat{\xi} \in \Gamma_{C R}(M, T M)$ is a CR-vector field on $M$. Then there exist an open neighborhood $U$ of $M$ in $X$ and a holomorphic vector field $\widehat{\zeta}$ on $U$ such that

$$
\widehat{\xi}(x)=\widehat{\zeta}(x)+\widehat{\zeta}^{\dagger}(x)
$$


for every $x \in M$, where $\widehat{\zeta}^{\dagger}$ denotes the complex conjugate of the vector field $\widehat{\zeta}$.

Definition: Let $M$ and $B$ be CR-manifolds. Suppose $(M, \pi, B, G)$ is a principal bundle, where $\pi: M \rightarrow B$ is the bundle projection and $G$ is the structure group. If the right action of $G$ on $M$ is by CR-transformations, then we call $(M, \pi, B, G)$ a CR-principal bundle.

\subsection{The Levi Form}

Suppose $(M, H)$ is a CR-manifold and let $\pi: T M \otimes \mathbb{C} \rightarrow T M \otimes \mathbb{C} / H \oplus \bar{H}$ denote the projection. Then $\pi$ induces an $\mathbb{R}$-linear bundle map

$$
L(M, H): H \times H \longrightarrow T M \otimes \mathbb{C} / H \oplus \bar{H}
$$

that is defined in the following way: For $x \in M$ and $(a, b) \in H_{x} \times H_{x}$ choose local sections $\widehat{\xi}$ and $\widehat{\zeta}$ in $H$ with $\widehat{\xi}(x)=a$ and $\widehat{\zeta}(x)=b$. Set

$$
L(M, H)(a, b):=\pi_{x}[\widehat{\xi}, \overline{\widehat{\zeta}}](x) .
$$

The map $L(M, H)$ is well defined and is called the Levi form of $(M, H)$. Some pertinent facts about the Levi form are:

1. The map $L$ is antihermitian.

2. The set $L^{\circ}:=\{\widehat{\xi} \in H \mid L(\widehat{\xi}, \widehat{\zeta})=0 \forall \widehat{\zeta} \in H\}$ is called the Levi kernel of $M$. We say that $L$ is non-degenerate if $L^{\circ}=\{0\}$.

3. If $M$ is a real hypersurface in a complex manifold $X$, then $i L$ has the same signature as the restriction of the complex Hessian matrix of a defining function of $M$ to $H=(T M \otimes \mathbb{C}) \cap T X^{1,0}$. The latter is the classical Levi form; see 11. Theorem, p. $290 \mathrm{ff}$ in [Gr].

4. A CR-manifold is called Levi flat if $L^{\circ}=H$.

\subsection{Homogeneous CR-structures}

Definition: A CR-automorphism of a given CR-manifold is a CR-map of $M$ to itself that is also a diffeomorphism. If the group of CR-automorphisms acts transitively on $M$, then $M$ is called a homogeneous CR-manifold. 
Theorem 2.3 ([Ri], Satz 1.3.1.2). Let $M=G / H$ be a homogeneous $C R$ manifold. The $G$-invariant $C R$-structures $(R, \mathcal{J})$ on $M$ are in one-to-one correspondence with the pairs $(\widetilde{R}, \widetilde{\mathcal{J}})$, where $\widetilde{R}$ is a vector subspace of $\mathfrak{g}$ with $\mathfrak{h} \subset \widetilde{R} \subset \mathfrak{g}$ and $\widetilde{\mathcal{J}}: \widetilde{R} \rightarrow \widetilde{R}$ is an endomorphism, that satisfy the following:

1) $\widetilde{\mathcal{J}} \xi=0$ if and only if $\xi \in \mathfrak{h}$

2) $\widetilde{\mathcal{J}}^{2} \xi+\xi \in \mathfrak{h}$ for all $\xi \in \widetilde{R}$

3) $\operatorname{Ad}_{g} \xi \in \widetilde{R}$ and $\operatorname{Ad}_{g} \widetilde{\mathcal{J}} \xi-\widetilde{\mathcal{J}} \operatorname{Ad}_{g} \xi \in \mathfrak{h}$ for all $g \in H$ and $\xi \in \widetilde{R}$

4) $[\xi, \zeta]-[\widetilde{\mathcal{J}} \xi, \widetilde{\mathcal{J}} \zeta] \in \widetilde{R}$ and $\widetilde{\mathcal{J}}([\xi, \zeta]-[\widetilde{\mathcal{J}} \xi, \widetilde{\mathcal{J}} \zeta])-[\widetilde{\mathcal{J}} \xi, \zeta]-[\xi, \widetilde{\mathcal{J}} \zeta] \in \mathfrak{h}$ for all $\xi, \zeta \in \widetilde{R}$.

Two pairs $(\widetilde{R}, \widetilde{\mathcal{J}})$ and $\left(\widetilde{R}^{\prime}, \widetilde{\mathcal{J}}^{\prime}\right)$ are equivalent if and only if $\widetilde{R}=\widetilde{R}^{\prime}$ and $\widetilde{\mathcal{J}} \xi-\widetilde{\mathcal{J}}^{\prime} \xi \in \mathfrak{h}$ for all $\xi \in \widetilde{R}=\widetilde{R}^{\prime}$. If $H$ is connected, then 3) may be replaced by

$$
\left.3^{\prime}\right)[\xi, \zeta] \in \widetilde{R} \text { and } \widetilde{\mathcal{J}}[\xi, \zeta]-[\xi, \widetilde{\mathcal{J}} \zeta] \in \mathfrak{h}
$$

for all $\xi \in \mathfrak{h}$ and $\zeta \in \widetilde{R}$.

The proof is given in [Ri], pp. 18 - 21, basically following [KN2] except $\widetilde{R} \neq \mathfrak{g}$.

Corollary 2.1 (Richthofer [Ri], Zusatz to Satz 2, p. 21). The G-invariant CR-structures on $M$ are analytic.

Consequence: Every homogeneous CR-manifold $M=G / H$ has a complexification.

\subsection{Complexification of the Lie algebra}

Let $M=G / H$ be a homogeneous CR-manifold. Since the invariant CRstructure $(R, \mathcal{J})$ on $G / H$ is analytic, a complexification $(\widehat{M}, \sigma)$ of $G / H$ exists. We will think of $M$ as already embedded in $\widehat{M}$, i.e., $M=\sigma(G / H)$. The action map $\lambda: G \times M \rightarrow M,(g, \sigma(a H)) \mapsto \sigma(g a H)$ induces a homomorphism $\phi: G \rightarrow \operatorname{Aut}_{C R}(M)$, the group of CR-automorphisms of $M$ and a homomorphism

$$
\alpha: \mathfrak{g} \longrightarrow \Gamma_{C R}(M, T M) .
$$

(Modulo ineffectivity, we are identifying $\mathfrak{g}$, the Lie algebra of $G$, with a subalgebra of the algebra of CR-vector fields on $M$.) For any $\xi \in \mathfrak{g}$, let 
$\left(\widehat{\xi}_{t}\right)_{t \in \mathbb{R}}$ be the one parameter group of the vector field $\alpha(X)$. Obviously, $\widehat{\xi}_{t}=\phi(\exp t \xi)$. Therefore, there exist a neighborhood $U$ of $M$ in $\widehat{M}$ and a holomorphic vector field $\widehat{\zeta}$ on $U$ such that $\widehat{\zeta}+\left.\overline{\widehat{\zeta}}\right|_{M}=\widehat{\xi}$, see Theorem 2.2, Since $\alpha(\mathfrak{g})$ is finite dimensional, one sees that by choosing a basis for this Lie algebra one can find an open neighborhood $U$ of $M$ such that every vector field $\widehat{\xi} \in \alpha(\mathfrak{g})$ can be so "extended" to a holomorphic vector field $\widetilde{\xi}$ on $U$. Moreover, the map $\xi \mapsto \widetilde{\xi}$ is a Lie algebra homomorphism. This construction allows us to define the complexification $\widehat{\mathfrak{g}}$ of the Lie algebra $\mathfrak{g}$ to be

$$
\widehat{\mathfrak{g}}:=\{\widetilde{\xi}+\mathcal{J} \widetilde{\zeta} \mid \xi, \zeta \in \mathfrak{g}\} ;
$$

namely, $\widehat{\mathfrak{g}}$ is the complex Lie subalgebra of $\Gamma\left(U, T^{1,0} U\right)$ that is generated by the image of $\mathfrak{g}$. One can easily check that $\mathfrak{m}:=\mathfrak{g} \cap \mathcal{J} \mathfrak{g}$ is an ideal in the Lie algebra $\mathfrak{g}$.

\subsection{The $G$-action on $\widehat{\mathfrak{g}}$}

We would now like to define a $G$-action on the complex Lie algebra $\widehat{\mathfrak{g}}$. In order to do this, we consider the action

$$
G \times \alpha(\mathfrak{g}) \longrightarrow \alpha(\mathfrak{g})
$$

given by

$$
g . \alpha(\xi)=\alpha\left(\operatorname{Ad}_{g} \xi\right)
$$

or

$$
g . \alpha(\xi)(p)=d \lambda_{g}\left(\alpha(\xi)\left(g^{-1} \cdot p\right)\right), \text { where } p \in M .
$$

If $\xi \in \mathfrak{m}$, then $\mathcal{J} \alpha(\xi)$ is tangential to $M$ and thus $g . \mathcal{J} \alpha(\xi)=\mathcal{J} g . \alpha(\xi)$, since $G$ is acting as a group of CR-transformations on $M$. One extends this action to $\widehat{\mathfrak{g}}$ in the following way. Let $\eta=\widetilde{\xi}+\mathcal{J} \widetilde{\zeta} \in \widehat{\mathfrak{g}}$. Define $g \eta=\widetilde{g \xi}+\mathcal{J} \widetilde{g \zeta}$, where $\widetilde{g \xi}$ is the extension of $g \cdot \alpha(\xi)$ and $g \zeta$ is the extension of $g \cdot \alpha(\zeta)$. Because of the complex linear $G$-action on $\alpha(\mathfrak{m})$, this extension is well-defined and the map $\eta \mapsto g . \eta$ of $\widehat{\mathfrak{g}} \rightarrow \widehat{\mathfrak{g}}$ is complex linear, i.e., $g . \mathcal{J} \eta=\mathcal{J} g . \eta$.

A naturally occurring situation is the following. Suppose $\widehat{G}$ is a complex Lie group and $\widehat{H}$ is a closed complex subgroup of $\widehat{G}$. Further, let $G$ be a real subgroup of $\widehat{G}$ such that $G / H \hookrightarrow \widehat{G} / \widehat{H}$ is a generic CR-submanifold, where $H=\widehat{H} \cap G$. Throughout this paper we will call the complex manifold $\widehat{M}:=$ 
$\widehat{G} / \widehat{H}$ a globalization of $M$, since the local action of $\widehat{G}$ in a neighborhood of $M$ has been globalized. Under the assumption that the actions are effective it follows that the Lie algebra $\widehat{\mathfrak{g}}$ of $\widehat{G}$ is isomorphic to the complexification of the Lie algebra $\mathfrak{g}$ of $G$ that was discussed above.

\subsection{The $\mathfrak{g}$-anticanonical fibration}

There are two main approaches to the definition of the $\mathfrak{g}$-anticanonical fibration. We will present both, beginning with a more algebraic method and then later using the complex tools at hand due to the embedding. Most of what follows can be found in [Ri], [HR], and [AHR].

\subsubsection{Equivariant CR-Fibrations}

If there exists a closed subgroup $I \subset G$ with $H \subset I$ such that $G / I$ has a $G$ invariant CR-structure and the projection $\pi: G / H \rightarrow G / I$ is a CR-mapping, then we will say that we have a CR-fibration. A fundamental question is whether such fibrations exist and what information they tell us about the homogeneous CR-manifold. Suppose the CR-structure on $M=G / H$ is given by a pair $(R, \mathcal{J})$ and the corresponding structure on $\mathfrak{g}$ is given by $(\widetilde{R}, \widetilde{\mathcal{J}})$, see Theorem 2.3 . Set

$$
N\left(H^{\circ}\right):=\left\{g \in G \mid g H^{\circ} g^{-1} \subset H^{\circ}\right\} .
$$

Then it is clear that $H \subset N\left(H^{\circ}\right)$ and at the Lie algebra level one has $N\left(H^{\circ}\right):=\left\{g \in G \mid \operatorname{Ad}_{g} \mathfrak{h} \subset \mathfrak{h}\right\}$. In order to take into account the CRstructure on $G / H$ as well, we consider the CR-normalizer $N_{C R}(H)$ of $H$ which is defined as follows:

$$
N_{C R}(H):=\left\{g \in G \mid \operatorname{Ad}_{g} \xi \in \widetilde{R} \text { and } \operatorname{Ad}_{g} \tilde{\mathcal{J}} \xi-\widetilde{\mathcal{J}} \operatorname{Ad}_{g} \xi \in \mathfrak{h}, \quad \forall \xi \in \widetilde{R}\right\} .
$$

Note that $N_{C R}(H)=N_{C R}\left(H^{\circ}\right)$ and the definition of $N_{C R}(H)$ only depends on the equivalence class of $(\widetilde{R}, \widetilde{\mathcal{J}})$, see Theorem 2.3 . By the same result $(\widetilde{R}, \widetilde{\mathcal{J}})$ also defines a CR-structure on $G / H^{\circ}$. Now one can define a real analytic right action

$$
r: G / H^{\circ} \times N\left(H^{\circ}\right) \longrightarrow G / H^{\circ}
$$

by $r\left(g H^{\circ}, n\right):=r_{n}\left(g H^{\circ}\right):=g n H^{\circ}$ for $g \in G$ and $n \in N\left(H^{\circ}\right)$. In general, the mapping $r_{n}$ is not a CR-mapping for every $n \in N\left(H^{\circ}\right)$. However, one has the following characterization of the subgroup $N_{C R}(H)$. 
Proposition 2.1 ([Ri] p. 23). One has $H \subset N_{C R}(H) \subset N\left(H^{\circ}\right)$. The group $N_{C R}(H)$ consists of all $n \in N_{G}\left(H^{\circ}\right)$ such that the right translations $r_{n}$ given by

$$
r_{n}: G / H^{\circ} \longrightarrow G / H^{\circ} \quad g H^{\circ} \mapsto g n H^{\circ}
$$

are CR-mappings.

Definition: The map $\varphi_{\widehat{\mathfrak{g}}}: G / H \rightarrow G / N_{C R}(H)$ is called the $\mathfrak{g}$-anticanonical fibration of $G / H$.

Remark: We note below that this map really is a CR-map. Also it is important to keep in mind that another construction of the $\mathfrak{g}$-anticanonical fibration is given in the next subsection, along with further details about some of its properties. The notation $\varphi_{\widehat{\mathfrak{g}}}$ is also justified by that construction, see 22.6 .2 .

An important consequence of the above that will be extremely useful later on is the following.

Corollary 2.2. If the $\mathfrak{g}$-anticanonical fibration is degenerate, i.e., $N_{C R}(H)=$ $G$, and the $G$-action is assume to be almost effective, then $H$ is discrete.

Suppose that $M$ is embedded in a complexification $\widehat{M}$. Let $\widehat{\mathfrak{h}}:=\{\zeta \in$ $\widehat{\mathfrak{g}} \mid \zeta(o)=0\}$, where $o=\pi(e)$ and $\pi: G \rightarrow G / H$. Recall

$$
\widehat{\mathfrak{m}}=\mathfrak{g}+\mathcal{J} \mathfrak{g}
$$

where $\mathcal{J}$ is the complex structure on $\widehat{M}$ and $\mathfrak{g}$ is regarded as a real subalgebra of $\Gamma_{\mathcal{O}}(\widehat{M}, T \widehat{M})$. It turns out that

$$
N_{C R}(H)=\left\{g \in G \mid \operatorname{Ad}_{g} \widehat{\mathfrak{h}}=\widehat{\mathfrak{h}}\right\},
$$

where $\operatorname{Ad}: G \rightarrow \operatorname{Aut}(\widehat{\mathfrak{g}})$ is the adjoint action of $G$ on $\widehat{\mathfrak{g}}$ that is induced by the usual adjoint action of $G$ on $\mathfrak{g}$. This shows that $G / N_{C R}(H)$ inherits a $G$-invariant CR-structure from the Grassmannian defined by the complex vector subspaces of $\widehat{\mathfrak{g}}$ that have the same dimension as $\widehat{\mathfrak{h}}$ and $N_{C R}(H)$ is the isotropy of the $G$-adjoint action computed at the point that corresponds to the subspace $\widehat{\mathfrak{h}}$. The Plücker embedding then gives us a $G$-equivariant map

$$
G / H \longrightarrow G / N_{C R}(H) \longrightarrow \mathbb{P}_{k} \text {. }
$$


This map is the same as the one given by the holomorphic sections of the anticanonical bundle of $\widehat{M}$ that are generated by the $\widehat{\mathfrak{g}}$-sections. This fact is the reason for the name $\mathfrak{g}$-anticanonical fibration.

We have a representation of $G$ into the group $G L_{k+1}(\mathbb{C})$. But we only use the action on the projective space, so we may regard $G$ as being mapped into $P G L_{k+1}(\mathbb{C})$. Let $\widetilde{G}$ denote the smallest complex Lie subgroup that contains the image of $G$ and let $\widetilde{N}_{C R}(H)$ denote the isotropy subgroup of the $\widetilde{G}$-action computed at the point corresponding to $N_{C R}(H)$.

Since $N_{C R}(H) \subset N_{G}\left(H^{\circ}\right)$, the fiber of the $\mathfrak{g}$-anticanonical fibration may be written as a quotient $A / \Gamma$, where $A:=N_{C R}(H) / H^{\circ}$ is a Lie group and $\Gamma:=H / H^{\circ}$ is a discrete subgroup of $A$. We will make some observations about this particular situation in 2.7.

The question whether $G / N_{C R}(H)$ admits a CR-structure such that the map $G / H \rightarrow G / N_{C R}(H)$ is a CR-submersion is answered by the following. Note that this result is slightly more general.

Theorem 2.4 ([Ri], Satz 1.3.2.2). Let $L \subset N_{C R}(H)$ be a closed subgroup such that $H \subset L$ and $\widetilde{\mathcal{J}}(\mathfrak{l} \cap \widetilde{R}) \subset(\mathfrak{l} \cap \widetilde{R})$, where $\mathfrak{l}$ denotes the Lie algebra of $L$. Then $G / L$ has a $G$-invariant $C R$-structure so that the map $G / H \rightarrow G / L$ is a CR-submersion.

Corollary 2.3. The manifold $G / N_{C R}(H)$ has a $G$-invariant $C R$-structure so that the map $G / H \rightarrow G / N_{C R}(H)$ is a CR-submersion.

In order to give a further description of the fibration $G / H \rightarrow G / N_{C R}(H)$ we now present some observations about the Levi form of invariant CRstructures $(R, \mathcal{J})$ on $M=G / H$.

Again let $\pi: G \rightarrow G / H$ be the quotient map and set $o=\pi(e)$. Let $\psi: \mathfrak{g} \rightarrow$ $\mathfrak{g} / \widetilde{R}$ be the projection. Set $\widetilde{L}(\xi, \zeta):=\psi[\xi, \zeta]$ for $\xi, \zeta \in \widetilde{R}$. Then

$$
\widetilde{L}=\left.\pi^{*} L_{R}\right|_{\widetilde{R}}
$$

This follows from the fact that $P(\widetilde{R})$ generates $\widetilde{R}_{g}$ at every point $g \in G$ and because $\widetilde{R}$ is a left invariant subbundle of $T G$. (For the notation, see Theorem 2.3.) 
Theorem 2.5 ([Ri], Satz 7). If $H$ is connected, then one has a CR-principal bundle

$$
G / H \longrightarrow G / N_{C R}(H) .
$$

Lemma 2.1 (Lemma 1, $\mathrm{AHR}$ ). If $\zeta \in \widehat{\mathfrak{g}}$ vanishes at a point $p \in M$, then $\zeta$ vanishes identically on $F_{p}$.

Corollary 2.4 ([AHR $)$. The fibers of $\varphi_{\mathfrak{\mathfrak { g }}}$ are Levi flat CR-submanifolds of $M$. If the image of $\varphi_{\widehat{\mathfrak{g}}}$ is a point, then

$$
\mathfrak{m}=\{\xi \in \mathfrak{g} \mid \mathcal{J} \widehat{\xi} \in \Gamma(M, T M)\}
$$

generates $L^{\circ}(M)$. If $M$ is a CR-hypersurface, then $\operatorname{dim}_{\mathbb{C}} \mathfrak{m}=n-1$, where $n:=\operatorname{dim}_{\mathbb{C}} \widehat{M}$.

If the image of $\varphi_{\mathfrak{\mathfrak { g }}}$ is a point, then $F_{p}=M$. Thus $\mathfrak{m}_{p} F:=\mathfrak{m}_{p}=\mathfrak{m}$. Since the $G$-action is almost effective, this observation suffices to complete the proof.

Corollary 2.5 (See [AHR]). Let $(M, G)$ be a homogeneous CR-manifold. Set $k:=\operatorname{rank}_{\mathbb{C}} L^{\circ}(M)$. Let $F$ be a fiber of the $\mathfrak{g}$-anticanonical fibration of $M$. Then $F$ is a Levi flat CR-submanifold of $M$ of type $(\alpha, \beta)$, where $\beta \leq k$. The ideal $\mathfrak{m}_{\mathfrak{j} / \mathfrak{h}}$ coming from the right action of $J / H^{\circ}$ on $G / H^{\circ}$ generates a complex subbundle of $L^{\circ}(M)$ of rank $\beta$.

\subsubsection{Another Construction and Further Properties}

Throughout we assume $G$ is connected and is acting almost effectively on $M$. Then the map $\alpha: \mathfrak{g} \rightarrow \Gamma(M, T M)$ that is induced by the action $\lambda$ : $G \times M \rightarrow M$ is injective. For $g \in G$, we write $\lambda_{g}: M \rightarrow M$ for the map $\lambda_{g}(x):=\lambda(g, x)$ and let $g \cdot x$ denote the point $\lambda(g, x)$.

Let $(\widehat{M}, \sigma)$ be a $\mathfrak{g}$-complexification of $M$ (see $₫ 2.4$ ) and $n=\operatorname{dim}_{\mathbb{C}} \widehat{M}$. In the following we will think of $M$ as already imbedded in $\widehat{M}$, i.e., $\left.\sigma\right|_{M}=\mathrm{id}_{M}$. Set

$$
V_{\mathfrak{g}}:=\bigwedge_{n} \widehat{\mathfrak{g}} .
$$

This is a $(k+1)$-dimensional complex vector subspace of the space of holomorphic sections of the anticanonical bundle of $\widehat{M}$ :

$$
V_{\mathfrak{g}} \subset \Gamma_{\mathcal{O}}\left(\widehat{M}, \bigwedge_{n} T \widehat{M}^{1,0}\right) .
$$


The natural $G$-action on $\widehat{\mathfrak{g}}$ (see 2.5 ) defines a $G$-action on $\mathbb{P}\left(V_{\mathfrak{g}}^{*}\right) \cong \mathbb{P}_{k}$ given by $g \cdot f(\sigma)=f\left(g^{-1} \cdot \sigma\right)$ for $g \in G, f \in \mathbb{P}\left(V_{\mathfrak{g}}^{*}\right), \sigma \in V_{\mathfrak{g}}$. The map $\mathbb{P}\left(V_{\mathfrak{g}}^{*}\right) \rightarrow \mathbb{P}\left(V_{\mathfrak{g}}^{*}\right), f \mapsto g \cdot f$ is projective linear for every $g \in G$; see $\$ 2.5$, Now let $U \subset \widehat{M}$ be an open neighborhood of $M$, so that for every $x \in U$ there exists a $\sigma \in V_{\mathfrak{g}}$ with $\sigma(x) \neq 0$, and let

$$
\widehat{\varphi_{\mathfrak{g}}}: U \longrightarrow \mathbb{P}\left(V_{\mathfrak{g}}^{*}\right)
$$

be given by $\widehat{\varphi_{\mathfrak{g}}}(x)(\sigma)=\sigma(x)$. Since $\bigwedge_{n} T \widehat{M}^{1,0}$ is a line bundle and for every $x \in U$ there exists a $\sigma \in V_{\mathfrak{g}}$ with $\sigma(x) \neq 0$, the map $\widehat{\varphi_{\widehat{\mathfrak{g}}}}$ is well defined. In order to show that $\widehat{\varphi_{\mathfrak{g}}}$ is also holomorphic, we pick a basis $\left\{\sigma_{0}, \ldots, \sigma_{k}\right\}$ of $V_{\mathfrak{g}}$ and set $\sigma=\sum_{i=0}^{k} a_{i}(\sigma) \sigma_{i}$. Then

$$
\widehat{\varphi_{\widehat{\mathfrak{g}}}}(x)(\sigma)=\sigma(x)=\sum_{i=0}^{k} a_{i}(\sigma) \sigma_{i}(x) .
$$

Let $\left\{\sigma_{0}^{*}, \ldots, \sigma_{k}^{*}\right\}$ be the dual basis of $V_{\mathfrak{g}}^{*}$ and let $\left[z_{0}: \ldots: z_{k}\right]$ be the homogeneous coordinates on $\mathbb{P}\left(V_{\mathfrak{g}}^{*}\right)$ that are defined by $\left\{\sigma_{0}^{*}, \ldots, \sigma_{k}^{*}\right\}$. Then any $f \in \mathbb{P}\left(V_{\mathfrak{g}}^{*}\right)$ can be written as $f=\left[z_{0}(f): \ldots: z_{k}(f)\right]$, where $z_{j}(f)=f\left(\sigma_{j}\right)$, for $j=0, \ldots, k$. Then

$$
z_{j}\left(\widehat{\varphi_{\widehat{\mathfrak{g}}}}(x)\right)=\widehat{\varphi_{\widehat{\mathfrak{g}}}}(x)\left(\sigma_{j}\right)=\sigma_{j}(x)
$$

and thus

$$
\widehat{\varphi_{\mathfrak{g}}}(x)=\left[\sigma_{0}(x): \ldots: \sigma_{k}(x)\right] .
$$

Since the $\sigma_{j} \in \Gamma_{\mathcal{O}}\left(\widehat{M}, \bigwedge_{n} T \widehat{M}^{1,0}\right)$, by the choice of $U$ one sees that $\widehat{\varphi_{\widehat{\mathfrak{g}}}}$ is holomorphic and well defined.

For $x \in \widehat{\varphi_{\mathfrak{g}}}(M)$, one has

$$
g \cdot \widehat{\varphi_{\widehat{\mathfrak{g}}}}(x)(\sigma)=\widehat{\varphi_{\widehat{\mathfrak{g}}}}(x)\left(g^{-1} \cdot \sigma\right)=[\sigma(g \cdot x)] .
$$

Thus in the coordinates chosen above

$$
g \cdot \widehat{\varphi_{\mathfrak{g}}}(x)=\left[\sigma_{0}(g \cdot x): \ldots: \sigma_{k}(g \cdot x)\right] .
$$

This implies that the map $\varphi_{\widehat{\mathfrak{g}}}:=\left.\widehat{\varphi_{\mathfrak{g}}}\right|_{M}$ is $G$-equivariant. Set

$$
J=\left\{g \in G: g \cdot \varphi_{\widehat{\mathfrak{g}}} \circ \pi(e)=\varphi_{\widehat{\mathfrak{g}}} \circ \pi(e)\right\},
$$

where $e$ is the identity in $G$ and $\pi: G \rightarrow G / H$ is the quotient map. Then $G / J$ is a (not necessarily closed) CR-submanifold of $\mathbb{P}_{k}$. 
Theorem 2.6. Let $G / H \rightarrow G / J$ be the fibration constructed above. Then $J=N_{C R}(H)$ (see (2.6.1) and if $G / N_{C R}(H)$ is endowed with its $G$-invariant $C R$-structure, then the map $G / N_{C R}(H) \rightarrow G / J$ is CR. In particular, $G / H \rightarrow$ $G / J$ is the $\mathfrak{g}$-anticanonical fibration of $G / H$.

Proof. First we note that

$$
J \subset N\left(H^{\circ}\right) .
$$

Otherwise, there would be an element $\xi$ in the Lie algebra $\mathfrak{h}$ of $H$ and an element $g \in J$ such that $\operatorname{Ad}_{g} \xi \notin \mathfrak{h}$. But then

$$
\eta(\pi(e)):=\frac{1}{2}(\widetilde{\xi}-i \mathcal{J} \widetilde{\zeta})(\pi(e))=0 \neq \eta(\pi(g))
$$

which contradicts the previous Lemma.

Since $(\widetilde{R}, \tilde{\mathcal{J}})$ (by the definition of $N_{C R}(H)$ in 92.6 .1 ) is defined by $\mathfrak{h}$ and $(R, \mathcal{J})$ only, one may consider the universal covering $G / H^{\circ}$ of $G / H$ with its lifted CR-structure and by Proposition 2.1 one has to show that $J$ consists of all elements $g \in N\left(H^{\circ}\right)$ such that the map $r_{g}: G / H^{\circ} \rightarrow G / H^{\circ}, a \cdot H^{\circ} \mapsto$ $a \cdot g H^{\circ}$ is $\mathrm{CR}$.

Let $g \in J$ and for $x \in G / H^{\circ}$ set $\mathfrak{m}_{x}=\left\{\widehat{\xi} \in \alpha(\mathfrak{g}): \xi(x) \in R_{x}\right\}$. Let $\widehat{\zeta}$ and $\widehat{\zeta^{\prime}}$ be the holomorphic vector fields on $U$ corresponding to $\widehat{\xi}$ and $\widehat{\xi^{\prime}}$. Then $\left(\widehat{\zeta^{\prime}}-i \widehat{\zeta}\right) \in \widehat{\mathfrak{g}}$ has a zero at the point $x$. By the preceding Lemma, this vector field vanishes along $F_{x}$. This means that $\mathcal{J} \widehat{\xi}=\widehat{\xi}^{\prime}$ on $F_{x}$. Thus $\mathfrak{m}_{x}$ generates the space $R_{p}$ for every $p \in F_{x}$. Moreover, for $g \in J$ one has

$$
\left.d r_{g}(\mathcal{J} \widehat{\xi}(x))=d r_{g} \widehat{\xi}^{\prime}(x)=\widehat{\xi}^{\prime}\left(r_{g}(x)\right)=\mathcal{J} \widehat{\xi}\left(r_{g}(x)\right)=\mathcal{J} d r_{g} \widehat{\xi}(x)\right),
$$

and so $r_{g}$ is a CR-map for every $g \in J$.

Conversely, if $g \in N_{C R}\left(H^{\circ}\right)$, then there exist neighborhoods $V$ and $W$ of $M$ in $U$ and an extension of $r_{g}$ to a biholomorphic map $\widetilde{r}_{g}: V \rightarrow W$. If $\eta:=\widetilde{\xi}+\mathcal{J} \widetilde{\zeta} \in \widehat{\mathfrak{g}}$, then on $W$ one has

$$
\widetilde{r_{g}} \cdot \widehat{\eta}(x)=d \widetilde{r}_{g} \widehat{\eta}\left(\widetilde{r}_{g}^{-1}(x)\right)=d \widetilde{r}_{g} \widetilde{\xi}\left(\widetilde{r}_{g}^{-1}(x)\right)+\mathcal{J} d \widetilde{r}_{g} \widetilde{\zeta}\left(\widetilde{r}_{g}^{-1}(x)\right)
$$

For $x \in M$ one has $d \widetilde{r}_{g} \widetilde{\xi}\left(\widetilde{r}_{g}^{-1}(x)\right)=\left(r_{g} \widehat{\xi}\right)(x)=\widehat{\xi}(x)$ for all $\widehat{\xi} \in \alpha(\mathfrak{g})$. Since the real holomorphic vector field $\left(\widetilde{r}_{g} \cdot \widetilde{\xi}\right)(x)=d \widetilde{r}_{g} \widetilde{\xi}\left(\widetilde{r}_{g}^{-1}(x)\right)$ is the continuation of 
$r_{g} \cdot \widehat{\xi}$ (and this also is true for $\left.\widetilde{r}_{g} \cdot \widetilde{\zeta}\right)$, it follows that $\widetilde{r}_{g} \cdot \widehat{\eta}=\widehat{\eta}$ on $W$, since $G / H^{\circ}$ is a generic CR-submanifold of $W$. In particular, one has

$$
\widehat{\eta}(g \cdot \pi(e))=\widehat{\eta}\left(r_{g} \cdot \pi(e)\right)=d \widetilde{r}_{g} \widehat{\eta}(\pi(e)) .
$$

Hence for $\sigma=\widehat{\eta}_{1} \wedge \ldots \wedge \widehat{\eta}_{n} \in V_{\mathfrak{g}}$ one has

$$
\sigma(g \cdot \pi(e))=d \widetilde{r}_{g} \widehat{\eta}_{1} \wedge \ldots \wedge d \widetilde{r}_{g} \widehat{\eta}_{n}(\pi(e))
$$

and thus

$$
\widehat{\varphi_{\mathfrak{g}}}(g \cdot \pi(e))(\sigma)=\widehat{\varphi_{\mathfrak{g}}}(\pi(e)) \sigma,
$$

and thus $g \in J$. This shows $J=N_{C R}(H)$. That the map $G / N_{C R}(H) \rightarrow G / J$ is CR follows from the fact that $G / H \rightarrow G / N_{C R}(H)$ is a CR-submersion and, because the map $\widehat{\varphi_{\widehat{\mathfrak{g}}}}$ is holomorphic, the map $\varphi_{\widehat{\mathfrak{g}}}$ is CR. (The CR-structure on $G / J$ contains $d \varphi_{\widehat{\mathfrak{g}}}(R)$ and is thus at most bigger than the structure on $G / N_{C R}(H)$.)

\subsection{Globalization of the Fiber}

The goal of this section is to prove the globalization result for a compact, homogeneous CR-manifold $G / H$ of codimension one and two under the assumption that the $\mathfrak{g}$-anticanonical fibration is degenerate, i.e., $N_{C R}(H)=G$. This implies that the isotropy subgroup $H$ is discrete. The proof is a straightforward modification of the proof of Satz 1.4.2.1 in [Ri].

Theorem 2.7 (Satz 1.4.2.1 [Ri]). Suppose $M=G / H$ is a generic homogeneous CR-manifold of codimension less than or equal to two. Let $\mathfrak{g}$ denote the Lie algebra of $G$. Assume that $G$ acts effectively on $M$ and the $\mathfrak{g}$-anticanonical fibration of $M$ is degenerate, i.e., $N_{C R}(H)=G$. There exists a simply connected, complex Lie group $\widehat{G}$ and a closed CR-embedding of the universal covering $\widetilde{G}$ of $G$ into $\widehat{G}$ as well as a discrete subgroup $\widetilde{\Gamma}$ of $\widetilde{G}$ such that $\widehat{G} / \widetilde{\Gamma}$ is a $\mathfrak{g}$-globalization of $\tilde{G} / \widetilde{\Gamma}=G / H$.

Proof. We let $\mathfrak{m}=\mathfrak{g} \cap \mathcal{J} \mathfrak{g}$ be the maximal complex ideal in $\mathfrak{g}$. Let $\widehat{M}$ be a $\mathfrak{g}$ complexification of $M$ and $\widehat{\mathfrak{g}}$ be the $\widehat{M}$-complexification of $\mathfrak{g}$. Further, let $\widehat{G}$ be the connected, simply connected, complex Lie group with Lie algebra $\widehat{\mathfrak{g}}$ that is uniquely determined up to isomorphism. We identify $\mathfrak{g}$ with $\{\widetilde{\xi} \mid \xi \in \mathfrak{g}\}$. Because the $\mathfrak{g}$-anticanonical fibration of $G / H$ is degenerate and because of the assumption on the codimension of the CR-structure on $M$, it follows that

$$
\operatorname{dim}_{\mathbb{C}} \widehat{\mathfrak{g}} \leq \operatorname{dim}_{\mathbb{C}} \mathfrak{m}+2
$$


Let

$$
\widehat{\mathfrak{g}}=\widehat{\mathfrak{r}}+\widehat{\mathfrak{s}} \quad \text { and } \quad \mathfrak{g}=\mathfrak{r}+\mathfrak{s}
$$

be a Levi decompositions of $\widehat{\mathfrak{g}}$ and $\mathfrak{g}$. Now $\widehat{\mathfrak{m}} \cap \widehat{\mathfrak{s}}$ is an ideal in $\widehat{\mathfrak{s}}$ and so by dimension reasons one has

$$
\widehat{\mathfrak{m}} \cap \widehat{\mathfrak{s}}=\widehat{\mathfrak{s}}
$$

and it follows from this that

$$
\widehat{\mathfrak{s}} \subset \widehat{\mathfrak{m}}=\mathfrak{m} \subset \mathfrak{g}
$$

In particular, $\widehat{\mathfrak{s}}=\mathfrak{s}$.

The radical $\mathfrak{r}$ of $\mathfrak{g}$ is obviously given by $\mathfrak{r}=\widehat{\mathfrak{r}} \cap \mathfrak{g}$. Let $\widehat{R}$ be the simply connected, complex Lie group with Lie algebra $\widehat{\mathfrak{r}}$ and $\widetilde{R}$ be the universal covering of the radical $R$ of $G$. Then $\widetilde{G}=\widetilde{R} \cdot S$, where $S$ denotes a (maximal) simply connected, semisimple, complex Lie group with Lie algebra $\mathfrak{s}=\widehat{\mathfrak{s}}$. The group $\widehat{G}$ has Levi-Malcev decomposition $\widehat{G}=\widehat{R} \rtimes S$. Since $\mathfrak{r}$ and $\widehat{\mathfrak{r}}$ are solvable, the inclusion $\mathfrak{r} \hookrightarrow \widehat{\mathfrak{r}}$ induces an imbedding $i: \widetilde{R} \hookrightarrow \widehat{R}$, see Chevalley's result Chev. The homomorphism $G \rightarrow \widehat{G}$ induced by the embedding $\mathfrak{g} \hookrightarrow \widehat{\mathfrak{g}}$ is given by

$$
\left(i, \operatorname{id}_{S}\right): \widetilde{R} \rtimes S=G \rightarrow \widehat{G}=\widehat{R} \rtimes S .
$$

Since $S$ contains a maximal, compact subgroup of $\widehat{G}$, the image of $G$ is closed in $\widehat{G}$, see Goto. Now let $\pi: \widetilde{G} \rightarrow G$ be the universal covering and set $\widetilde{\Gamma}:=\pi^{-1}(H)$. Since $G$ acts effectively on $G / H$ and the $\mathfrak{g}$-anticanonical fibration is degenerate, $H$ is discrete in $G$ and thus $\widetilde{\Gamma}$ is discrete in $\widetilde{G}$. Since $G \subset \widehat{G}$ is closed, $\widetilde{\Gamma} \subset \widehat{G}$ is closed and $\widehat{G} / \widetilde{\Gamma}$ is obviously a $\mathfrak{g}$-globalization of $\widetilde{G} / \widetilde{\Gamma}=G / H$. It is clear that the embedding $\widetilde{G} \hookrightarrow \widehat{G}$ is CR.

\section{Globalization}

Our goal here is to derive a condition under which the local action of $\widehat{G}$ in a neighborhood of the CR-homogeneous manifold $M$ can be globalized. By this we mean that there is a complex homogeneous manifold $X=\widehat{G} / \widehat{H}$ with $M$ being the $\mathrm{CR}$-equivariantly equivalent to the $G$-orbit of its neutral point. Here we do not assume that $M$ is compact, but otherwise we operate under the usual assumptions and notation of this paper. In particular, $G$ is asssumed to be embedded in the simply-connected complex Lie group $\widehat{G}$. 
As the following example shows, in order to achieve globalization it might be necessary to modify $M$ (see, e.g., $[\mathrm{HR}$ ] for more details and [KZ for much more general considerations). In Section 5 we will provide examples where the necessary modifications are much more serious. On the other hand, we use there the criteria developed in the present section to show that "most" $M$ can be globalized without significant modifications.

Example. Consider the 2-dimensional affine quadric $X:=\widehat{G} / \widehat{J}$, where $\widehat{G}=\mathrm{SL}_{2}(\mathbb{C})$ and $\widehat{J}$ is the subgroup of diagonal matrices. Note that $\widehat{J}$ contains the group consisting of \pm Id so that $\widehat{G}$ acts with this small ineffectivity. Fix $x_{0} \in X$ as a neutral point where $\widehat{J}=\widehat{G}_{x_{0}}$. The unipotent group $\widehat{U} \cong \mathbb{C}$ of upper-triangular matrices realizes $X$ as the total space of the principal $\mathbb{C}$-bundle $\widehat{G} / \widehat{J} \rightarrow \widehat{G} / \widehat{J} \widehat{U} \cong \mathbb{P}_{1}(\mathbb{C})$. Identifying the $\widehat{U}$-orbit $\widehat{U} \cdot x_{0}$ with $\mathbb{C}$, define $\Sigma$ to be the subset of $X$ which corresponds $\mathbb{R}^{\geq 0}$.

Now let $G=\mathrm{SU}_{2}$ and observe that every $G$-orbit in $X$ intersects the slice $\Sigma$ in exactly one point. The $G$-orbit $M_{x_{0}}$ of the neutral point is a copy of the 2-sphere which is embedded as a totally real submanifold. Otherwise for all $x \in \Sigma \backslash\left\{x_{0}\right\}$ the orbit $M_{x}=G . x$ is a hypersurface. Since $G_{x}$ is just the ineffectivity mentioned above, $M_{x}$ is simply the group $\mathrm{PSU}_{2}$ equipped with a left-invariant $\mathrm{CR}$-structure.

Consider the universal cover $\tilde{Z}$ of the complement $Z$ of $M_{x_{0}}$ in $X$. Here $G$ acts freely as a group of holomorphic transformations. The slice $\Sigma$ lifts to a slice $\tilde{\Sigma}$ for the $G$-action. For $\tilde{x} \in \tilde{\Sigma}$ the CR-homogeneous space $\tilde{M}_{x}$ is just the group $G$ equipped with a left-invariant $\mathrm{CR}$-structure. It is an example of a strongly pseudoconvex hypersurface which can not be filled in to a Stein space. It also can not be globalized in our sense, because if there would be a globalization $\widehat{G} / \widehat{H}$, then $\widehat{H}$ would be a subgroup of $\widehat{J}$ and this would force $\widehat{H}=\widehat{J}$ so that $\tilde{M}_{x}=M_{x}$.

\subsection{Homogeneous fibrations}

Here we provide a criterion for the existence of a globalization of the total space of a homogeneous fiber bundle where it is known that the fiber and base are globalizable. As usual the connected complex Lie group $\widehat{G}$ is assumed to be simply connected and $G$ is a connected, real (not necessarily totally real) subgroup of $\widehat{G}$ with $\widehat{\mathfrak{g}}=\mathfrak{g}+i \mathfrak{g}$. We assume that the homogeneous 
CR-manifold $M=G / H$ is the total space of a $G$-homogeneous fiber bundle $\pi: G / H \rightarrow G / J$ the base of which is the $G$-orbit of the neutral point in a complex homogeneous manifold $\widehat{G} / \widehat{J}$. The map $\pi$ is assumed to be holomorphic and locally $\widehat{G}$-equivariant in some neighborhood of $M$. The connected component $J^{\circ} \mathrm{H} / \mathrm{H}$ of the fiber $J / H$ is denoted by $F$.

If the fiber $F$ possesses a $\widehat{J}$-globalization $\widehat{F}$, then one is naturally led to consider the complex $\widehat{G}$-manifold $\widehat{M}:=\widehat{G} \times_{\widehat{J}} \widehat{F}$. The $G$-orbit of the neutral point in $\widehat{M}$ is indeed the CR-homogeneous space $M=G / H$.

In applications one can at most hope that $\widehat{F}$ is a complex manifold equipped with a local holomorphic action of $\widehat{J}^{0}$. Thus we at first assume that $\widehat{J}$ is connected, an assumption that can be realized by going to a $G$-equivariant covering space of $M$. In the end this turns out to be no assumption at all. Even though $F$ might not be connected, since $\widehat{J}$ is connected, we must assume that $\widehat{F}$ is connected. So, given $F$ and $\widehat{F}$, we must replace them by their connected components. Finally, we assume that the holomorphic vector fields coming from the $\widehat{J}$-action on $\widehat{F}$ can be integrated so that the universal cover $\widehat{J}_{1}$ of $\widehat{J}$ acts holomorphically on $\widehat{F}$. If all of these assumptions are satisfied, we say that the fiber and base of the CR-homogeneous bundle $G / H \rightarrow G / J$ are globalizable. The following yields a first criterion for a $\hat{G}$-globalization.

Proposition 3.1. If the inclusion $J^{\circ} \hookrightarrow \widehat{J}$ induces a surjective map of fundamental groups, then the $\widehat{J}_{1}$-action on $\widehat{F}$ descends to a $\widehat{J}$-action.

Proof. If $J_{1}^{\circ}$ is the lift of $J^{\circ}$ into $\widehat{J}_{1}$, then the condition on surjectivity of the fundamental groups implies that the kernel $\Lambda$ of the map $J_{1}^{\circ} \rightarrow J^{\circ}$ is the same as that of $\widehat{J}_{1} \rightarrow \widehat{J}$. Since $\Lambda$ acts trivially on $\widehat{F}$, the action of $\widehat{J}_{1}$ descends to that of $\widehat{J}_{1} / \Lambda=\widehat{J}$.

In our applications we are only able to answer such homotopy questions modulo the ineffectivity of the actions on the base manifold. Thus a useful criterion should be given at that level. For this we let $\widehat{I}$ be the ineffectivity of the $\widehat{G}$-action on $\widehat{G} / \widehat{J}$ and $I=\widehat{I} \cap G$ the $G$-ineffectivity. For notational simplicity, let us refer to the following as

\section{Condition (C):}


- The inclusion $J^{\circ} /\left(I \cap J^{\circ}\right) \hookrightarrow \widehat{J} / \widehat{I}$ induces a surjective map of fundamental groups.

Proposition 3.2. If

$$
J^{\circ} \cap \widehat{I}^{\circ}=I^{\circ},
$$

then condition $(C)$ implies that the $\widehat{J}_{1}$ action on $\widehat{F}$ descends to a $\widehat{J}$-action.

Proof. Condition (11) implies that there is a natural commutative diagram defined by the homotopy sequences associated to the fibrations

$$
\widehat{I}^{\circ} \rightarrow \widehat{J} \rightarrow \widehat{J} / \widehat{I}^{\circ} \text { and } I^{\circ} \rightarrow J^{\circ} \rightarrow J^{\circ} / I^{\circ}
$$

Now $\widehat{I}^{\circ}$ is simply connected, because it is a normal, connected subgroup of a simply connected Lie group. Thus, using the above mentioned homotopy sequences, in order to show that the inclusion $J^{\circ} \hookrightarrow \widehat{J}$ induces a surjective map of fundamental groups, we must only show that

$$
\pi_{1}\left(J^{\circ} / I^{\circ}\right) \rightarrow \pi_{1}\left(\widehat{J} / \widehat{I}^{\circ}\right)
$$

is surjective. We claim that this follows from condition (C).

For this observe that if $\gamma$ represents a homotopy class in $\widehat{J} / \widehat{I}^{\circ}$ with a base point in $J^{\circ} / I^{\circ}$, then the given surjectivity implies that there is a homotopy of its image in $\widehat{J} / \widehat{I}$ to a closed curve in $J^{\circ} / J^{\circ} \cap I$. Since this homotopy can be lifted to a homotopy of $\gamma$ to a curve in $J^{\circ} / I^{\circ}$, the desired result follows.

The following presents a situation where Proposition $\underline{3.2}$ can be applied.

Proposition 3.3. If the radical $\widehat{R}$ of $\widehat{G}$ is acting on $\widehat{G} / \widehat{J}$ as an Abelian group, i.e., if $\widehat{J}$ contains the commutator group $\widehat{R}^{\prime}$, then (1) in Proposition 3.2 is satisfied.

The following Lemma is the general fact behind this result.

Lemma 3.1. If $\widehat{N}$ is a connected, complex normal subgroup of $\widehat{G}$ which contains the commutator subgroup $\widehat{R}^{\prime}$, then $\widehat{N} \cap G$ is connected. 
Proof. At the Lie algebra level we have $\widehat{\mathfrak{r}}^{\prime}=\mathfrak{r}^{\prime}+i \mathfrak{r}^{\prime}$. Thus $\widehat{R}^{\prime} \cap G=R^{\prime}$. Consequently, $\widehat{N} \supset R^{\prime}$ and it is sufficient to prove the result in the case where $\widehat{R}$ is Abelian. Now in general $\widehat{N} \cap \widehat{R}$ is just the radical $\widehat{R}_{\widehat{N}}$. In the Abelian case this is a vector subspace of $\widehat{R}$. Since the same is true of $R$, it follows that $\widehat{N} \cap R$ is connected. Thus it is only necessary to show that $\widehat{N} / \widehat{R}$ has connected intersection with the image of $G$ in $\widehat{G} / \widehat{R}$.

For this it is convenient to consider a Levi-Malcev decomposition $G=R \rtimes S$ which lines up with a Levi-Malcev decomposition $\widehat{G}=\widehat{R} \rtimes \widehat{S}$. By this we simply mean that $S \subset \widehat{S}$. Thus the simple factors of $S$ are either complex simple factors of $\widehat{S}$, or real forms of complex simple factors of $\widehat{S}$ or antiholomorphic diagonals of products of two isomorphic simple factors of $\widehat{S}$. Since we may identify the image of $G$ with $S$ in the quotient $\widehat{G} / \widehat{R}=\widehat{S}$ and $\widehat{N} / \widehat{R}$ with a product of certain of the simple factors of $\widehat{S}$, the desired result follows.

Proof of Proposition 3.3. The lemma shows that $\widehat{I}^{0} \cap G=I^{0}$ and, since $I^{0} \subset J^{0}$, condition (1) of Proposition 3.2 is fulfilled.

\subsection{Remarks on coverings}

Here we at first continue under the assumptions of the previous paragraph, in particular that $\widehat{J}$ is connected. The work there shows the following.

Proposition 3.4. If $\widehat{R}$ is acting as an Abelian group on $\widehat{G} / \widehat{J}$ and condition (C) is fulfilled, then $\widehat{J}$ acts holomorphically on $\widehat{F}$.

In this situation we therefore consider the $G$-orbit $G / \tilde{H}$ of the neutral point in the complex $\widehat{G}$-manifold $\widehat{G} \times \widehat{J} \widehat{F}=\widehat{G} / \widehat{H}$. Now by construction $H$ fixes this neutral point; so $\tilde{H} \supset H$. Furthermore, $G / \tilde{H}$ is an $F$-bundle over $G / J$ and consequently $\operatorname{dim} G / H=\operatorname{dim} G / \tilde{H}$. Therefore $M=G / H \rightarrow G / \tilde{H}=\tilde{M}$ is a covering. Hence, in the setting above the previous paragraph, if condition (C) is fulfilled and $\widehat{R}$ acts as an Abelian group on $\widehat{G} / \widehat{J}$, then after replacing $M$ by a discrete $G$-equivariant quotient, the local $\widehat{G}$ action near $M$ can be globalized.

The example in the previous section shows that a discrete quotient may in fact be necessary. In that case it is just the quotient that maps the given nonglobalizable manifold to the hypersurface orbit in the affine quadric. 
There is a covering which has been implicitly used above and which is actually not necessary for globalization. This occurs as follows. Let us not assume that $\widehat{J}$ is connected. Then, abusing the notation which was used above, we let $H_{1}:=H \cap \widehat{J}^{\circ}$ and $J_{1}:=J \cap \widehat{J}^{\circ}$. Having done this, we may apply the above results. If globalization conditions are satisfied and $\widehat{G} / \widehat{H}_{1}$ is the resulting complex $\widehat{G}$-homogeneous manifold, then it is possible to return to the original situation. For this note that the fiber $F$ of the resulting map $G \times_{H_{1}} F \rightarrow G / J_{1}$ is just the connected component of the fiber $J / H$. In particular $H$ fixes the neutral point in $F$ and consequently $H \subset \widehat{H}_{1}$. If we then return to the original situation by replacing $\widehat{J}^{\circ}$ by the original complex group $\widehat{J}$ we have the desired globalization, of course with a possibly disconnected fiber. Let us now formulate this result for future reference. For simplicity we bundle together the assumptions of the

\section{Standard Situation:}

- The complex Lie group $\widehat{G}$ is connected and simply connected.

- The real (not necessarily totally real) subgroup $G$ is connected with $\mathfrak{g}+i \mathfrak{g}=\widehat{\mathfrak{g}}$.

- $M=G / H$ is a homogeneous CR-manifold with $\widehat{G}$ acting locally holomorphically on a complex manifold which is a local neighborhood of $M$.

- $G / H \rightarrow G / J$ is a CR-homogeneous fiber bundle.

- The base $G / J$ is the $G$-orbit of the neutral point in a $\widehat{G}$-homogeneous space $\widehat{G} / \widehat{J}$.

- The connected component $F$ of the fiber $J / H$ possesses a (connected) globalization $\widehat{F}$ on which the universal cover $\widehat{J}_{1}$ of the connected component $\widehat{J}^{\circ}$ holomorphically acts.

Theorem 3.1. If in the standard situation the radical $\widehat{R}$ acts as an Abelian group on $\widehat{G} / \widehat{J}$ and condition $(C)$ is fulfilled, then $\widehat{J}^{\circ}$ acts holomorphically on $\widehat{F}$ and there exists a globalization $\widehat{G} / \widehat{H} \rightarrow \widehat{G} / \widehat{J}$ of the CR-bundle $G / \tilde{H} \rightarrow$ $G / J$, where $M:=G / H \rightarrow G / \tilde{H}=\tilde{M}$ is a covering. 
Note that in the case where $M$ is compact the covering $M \rightarrow \tilde{M}$ is at most finite-fibered. This is a small price to pay for a globalization.

In situations where we wish to apply Theorem 3.1 it is quite often only possible to verify a weaker version of condition $(\mathrm{C})$, namely that the inclusion $J^{0} /\left(I \cap J^{0}\right) \hookrightarrow \widehat{J} / \widehat{I}$ induces a map of fundamental groups with the property that the image of $\pi_{1}\left(J^{0} /\left(I \cap J^{0}\right)\right)$ has finite index in $\pi_{1}(\widehat{J} / \widehat{I})$. We now replace condition $(\mathrm{C})$ by this weaker version and note the following

Zusatz. Theorem 3.1 holds under the weakened version of condition $(C)$.

Proof. If only the weakened version of conditon (C) holds, then the above shows that nevertheless a finite covering space $\widehat{J}_{1}$ of $\widehat{J}$ acts (transitively) on $\widehat{F}$. Let us write $\widehat{F}=\widehat{J}_{1} / \widehat{I}$ and denote by $\Gamma$ the kernel of $\widehat{J}_{1} \rightarrow \widehat{J}$. It is a finite central subgroup of $\widehat{J}_{1}$. If we replace $\widehat{F}$ by $\widehat{F}_{1}:=\widehat{J}_{1} / \Gamma \widehat{I}$, then $\widehat{J}$ acts on $\widehat{F}_{1}$ and we have the globalization $\widehat{G} \times \widehat{J}_{\widehat{F}}$. The $G$-orbit of the neutral point in this manifold is perhaps a finite quotient of the original manifold $G / H$, but finite quotients are allowed in the statement of Theorem 3.1

\subsection{The case of the $\mathfrak{g}$-anticanonical fibration}

Our main application of the globalization criterion is in the case of the $\mathfrak{g}^{-}$ anticanonical fibration $M:=G / H \rightarrow G / J \hookrightarrow \widehat{G} / \widehat{J}$. Here we let $\ell$ be the real Lie algebra $\mathfrak{j} / \mathfrak{h}$ and $\widehat{\ell}:=\widehat{j} / \widehat{h}$. If $L$ and $\widehat{L}$ are the associated groups, where as in our general setup $\widehat{L}$ is taken to be simply connected, then $F=L / \Gamma$, where $\Gamma$ is discrete. If the local $\widehat{L}$-action can be globalized to holomorphically act on $\widehat{F}$ as in the standard assumptions, then the globalization criterian can be applied.

Theorem 3.2. Let $M=G / H \rightarrow G / J \hookrightarrow \widehat{G} / \widehat{J}$ be the $\mathfrak{g}$-anticanonical fibration of the homogeneous CR-manifold $M$. Suppose that the connected component $F=L / \Gamma$ of the fiber possesses an $\widehat{L}$ globalization $\widehat{F}$. Then, if $\widehat{R}$ acts as an Abelian group on $\widehat{G} / \widehat{J}$ and condition $(C)$ is fulfilled, $\widehat{J}^{\circ}$ acts holomorphically on $\widehat{F}$ and there exists a globalization $\widehat{G} / \widehat{H} \rightarrow \widehat{G} / \widehat{J}$ of the CR-bundle $G / \tilde{H} \rightarrow G / J$, where $M:=G / H \rightarrow G / \tilde{H}=\tilde{M}$ is a covering. 


\section{Structure theorem in the projective case}

In the previous section we gave a criterion for the existence of a $\widehat{G}$-globalization of a CR-homogeneous space $M$. To apply this criterion one needs explicit knowledge of certain properties of the base of the $\mathfrak{g}$-anticanonical fibration. The main purpose of this section is to prove the first of these properties, namely, that the radical $\widehat{R}$ of the group $\widehat{G}$ acts as an Abelian group on the globalization $\widehat{G} / \widehat{J}$ of the base $G / J$ of the $\mathfrak{g}$-anticanonical fibration. We emphasize that there is no restriction on the codimension of the CR-structure on $G / J$ for this to hold. The other property one needs in order to apply Theorem 3.2 is condition (C) and settings where condition (C) is fulfilled are discussed in the next section.

The base of the $\mathfrak{g}$-anticanonical fibration is itself a CR-homogeneous space which is a $G$-orbit in the projective space $\mathbb{P}(V)$ of a $\hat{G}$-representation space $V$. In the notation of the previous section we have the globalization

$$
G / J \hookrightarrow \widehat{G} / \widehat{J} \hookrightarrow \mathbb{P}(V)
$$

We prove here a structure theorem that gives a first description of this situation. It should be underlined that the group $\widehat{G}$ is only represented on $V$; in particular it may be acting with ineffectivity and both $G$ and $\widehat{G}$ are possibly not closed in $\mathrm{PGL}_{\mathbb{C}}(V)$. Although the globalization criterion requires information about the $G$-action, we nevertheless replace $G$ by the closure of its image in $\mathrm{PGL}_{\mathbb{C}}(V)$. Since we are concerned here with compact $\mathrm{CR}-$ homogeneous spaces, this closure stablizes the base of the $\mathfrak{g}$-anticanonical fibration. Using the structure theorem proved here and the detailed classification results of the next section, we then recapture enough information about the original group in order to apply the globalization theory.

A complex Lie group is called reductive if it is the complexification of a maximal compact subgroup. One should recall that reductive Lie groups always carry the structure of linear algebraic groups.

Theorem 4.1. Let $G$ be a connected real (not necessarily totally real) closed Lie subgroup of $\mathrm{PGL}_{\mathbb{C}}(V)$ and $M=G . x_{0}$ be a compact orbit in $\mathbb{P}(V)$ with $\mathbb{P}(V)$ assumed to be the projective linear hull of $M$. Let $\widehat{G}$ be the smallest complex Lie group containing $G$ in $\mathrm{PGL}_{\mathbb{C}}(V)$, i.e., the group corresponding to the Lie algebra $\widehat{\mathfrak{g}}=\mathfrak{g}+i \mathfrak{g}$. Denote the complex algebraic closure of $G$ by 
$\bar{G}$. Let $G=R \cdot S$ denote a Levi-Malcev decomposition of $G$ and $\widehat{G}=\widehat{R} \cdot \widehat{S}$ a Levi-Malcev decomposition of $\widehat{G}$. Then $R$ is central, compact, acts freely on $M$, and is totally real with complexification $\widehat{R} \cong\left(\mathbb{C}^{*}\right)^{k}$. The group $\widehat{G}=\widehat{S} \cdot \widehat{R}$ is reductive, and hence algebraic, and thus $\widehat{G}=\bar{G}$. Moreover, any maximal compact subgroup of $G$ acts transitively on $M$.

The result follows from a number of observations that we now give.

Proposition 4.1. The radical $R$ of $G$ is Abelian.

Proof. The commutator group $R^{\prime}$ is a real unipotent group and is in particular a real algebraic subgroup of $\operatorname{PGL}_{\mathbb{C}}(V)$. Thus, on the boundary of every $R^{\prime}$-orbit in $\mathbb{P}(V)$ there are only $R^{\prime}$-orbits of lower-dimension. Furthermore, every $R^{\prime}$-orbit is algebraically diffeomorphic to some $\mathbb{R}^{m}$. In particular, the only possibility for a closed orbit is a fixed point. Since $R^{\prime}$ is a normal subgroup of $G$, it follows that $G$ acts transitively on the set of $R^{\prime}$-orbits in $M$. In particular, the $R^{\prime}$-orbits in $M$ are equidimensional and since $M$ is compact, they must all be closed, i.e. $R^{\prime}$ fixes $M$ pointwise. Since $\mathbb{P}(V)$ is the projective linear hull of $M$, it follows that $R^{\prime}=\{\mathrm{Id}\}$.

Remark. It should be noted that the process of replacing the original group $G$ by the closure of its representation on $\mathbb{P}(V)$ only enlarges the radical. Thus the above result guarantees that the radical of the original group is acting as an Abelian group on $\mathbb{P}(V)$.

Since $R$ is Abelian, it has a unique maximal compact subgroup $T$, i.e., its maximal compact torus. We will now show that in fact $R=T$. Note that since $T$ is stabilized by conjugation by $S$ and the group-theoretic automorphism group of $T$ is discrete, it follows that $T$ is a central subgroup of $G$.

Proposition 4.2. The radical $R$ is a central subgroup of $G$.

Proof. The radical $R$ (analytically) decomposes into a $G$-invariant product $R=T \times V$, where $V$ is the additive group of a vector space. Since $T$ is central, $R_{G^{\prime}} \subset V$. By Chevalley's Theorem $G^{\prime}$ is a real algebraic group and therefore so is $R_{G^{\prime}}$. Unless it is trivial, it is noncompact with all orbits noncompact. Since $R_{G^{\prime}}$ is a normal subgroup of $G$, all of its orbits in $M$ have the same dimension. Therefore the same argument as that above which showed that $R^{\prime}$ is trivial shows here that $R_{G^{\prime}}$ is trivial and therefore $R$ is central. 
Corollary 4.1. The radical of $G$ is a compact torus, i.e., $R=T$.

Proof. Since $R$ is central in $G$, the radical $\bar{R}$ of the algebraic closure $\bar{G}$ is central in $\bar{G}$. Thus, for any two points $x, y \in \bar{G} \cdot x_{0}$ the isotropy groups $\bar{R}_{x}$ and $\bar{R}_{y}$ agree. Since the linear hull of $M$ is the full space $\mathbb{P}(V)$ and $\bar{G} \cdot x_{0} \supset M$, it follows that $\bar{R}$ acts freely on $\bar{G} \cdot x_{0}$. Since $\bar{R}$ is an normal algebraic subgroup of $\bar{G}$, its orbits in $\bar{G} \cdot x_{0}$ are closed. Now $R$ is a closed subgroup of $\bar{R}$. So all of its orbits are closed in $\bar{G} \cdot x_{0}$ as well; in particular, its orbits in $M$ are closed in $M$. But $R$ is acting freely on $M$ and therefore $R=T$.

Since $T$ is a compact torus in a linear group, it is totally real and therefore $\widehat{T}=T^{\mathbb{C}} \cong\left(\mathbb{C}^{*}\right)^{m}$. It follows that $\widehat{G}=\widehat{T} \widehat{S}$ is reductive and therefore $\widehat{G}=\bar{G}$.

Note that since $G$ is the product $G=T \cdot S$ of a compact torus and a semisimple group, it is a (real, but not necessarily totally real) algebraic subgroup of $\mathrm{PGL}_{\mathbb{C}}(V)$. Thus its isotropy group $G_{x_{0}}$ has only finitely many components.

Corollary 4.2. Every maximal compact subgroup $K=T \cdot K_{S}$ of $G$ acts transitively on $M$.

Proof. Since $M$ is compact and $G_{x_{0}}$ has only finitely many components, Montgomery's theorem [Mont] guarantees this.

Quotienting out by $\widehat{T}$ leads to the following picture:

$$
\begin{aligned}
& M=G / H \hookrightarrow \widehat{G} / \widehat{H} \hookrightarrow \mathbb{P}(V) \\
& \downarrow \quad \downarrow \\
& N=G / T H \hookrightarrow \widehat{G} / \widehat{T} \widehat{H} \hookrightarrow \mathbb{P}(W)
\end{aligned}
$$

Here $G / H \rightarrow G / T H$ and $\widehat{G} / \widehat{H} \rightarrow \widehat{G} / \widehat{T} \widehat{H}$ are principal $T$ - and $\widehat{T}$-bundles. Since $\widehat{G} / \widehat{H} \rightarrow \widehat{G} / \widehat{T} \widehat{H}$ is a quotient in an algebraic group setting, as the notation indicates the base is therefore equivariantly embedded as a $\hat{G}$-orbit in a projective space.

Now $S$ acts transitively on $N$ and is acting algebraically on $M$. Furthermore, it is a normal subgroup of $G$. Hence by the same argument that has been applied several times above, the $S$-orbits in $M$ are also compact. We regard $S . x_{0}$ as a thick section for the fibration $M=G / H \rightarrow G / T H=S / I_{S}=N$. The connected component of the fiber of the map $\Sigma:=S . x_{0}=S / H_{S} \rightarrow S / I_{S}$ can be regarded as a subtorus $T_{0}$ of $T$. If $T_{1}$ in a complementary torus to $T_{0}$ in $T$, then we have the following observation. 
Proposition 4.3. The map $T_{1} \times \Sigma \rightarrow M$ defined by the action by $T_{1}$ and the canonical injection of $\Sigma$ realizes $M$ as a finite quotient $T_{1} \times_{\Gamma} \Sigma$.

Thus up to finite quotients the CR-homogeneous space is the product of the totally real torus $T_{1}$ and the CR-homogeneous space $\Sigma$. This product stucture may not be the optimal one from the Cauchy-Riemann viewpoint, because at the complexified level the bundle $\widehat{G} / \widehat{H} \rightarrow \widehat{G} / \widehat{T}, \widehat{H}$ might not split accordingly. For example, $\widehat{S}$ could act transitively on $\widehat{G} / \widehat{H}$ !

Using the fact that $S$ is a normal subgroup of $G$ we are able split off the maximal complex subgroup of $G$ so that in all future considerations of projective CR-homogeneous spaces we may assume that $G$ is a real form of $\widehat{G}$. For this we let $\mathfrak{l}=\mathfrak{g} \cap i \mathfrak{g}$ be the ideal which defines this subgroup at the Lie algebra level. Since $\mathfrak{g}$ is the Lie algebra direct sum $\mathfrak{g}=\mathfrak{t} \oplus \mathfrak{s}$ and $\mathfrak{t}$ is totally real in $\widehat{\mathfrak{g}}$, it follows that $\mathfrak{l}$ is the sum of the simple summands of $\mathfrak{s}$ which are simple summands of $\widehat{\mathfrak{s}}$. Thus the associated complex group $L$ is a product of the factors of $S$ which are also factors of $\widehat{S}$. Since $L=\widehat{L}$ is an algebraic normal subgroup of $G$ which is acting algebraically on the compact manifold $M$, its orbits are all isomorphic to a fixed compact projective algebraic homogeneous space $Z$ (an $L$-flag manifold).

Proposition 4.4. The complex algebraic bundle $\widehat{G} / \widehat{H} \rightarrow \widehat{G} / \widehat{H} \widehat{L}$ is $\widehat{G}^{-}$ equivariantly trivial.

Proof. The $\widehat{L}$-isotropy group $\widehat{P}$ at $x_{0}$ has exactly one fixed point in the flag manifold $Z=\widehat{L} / \widehat{P}$. Thus the same is true of every $\widehat{L}$-orbit in $\widehat{G} / \widehat{H}$. Consequently the fixed point set $\mathcal{F}:=\operatorname{Fix}(\widehat{P})$ of $\widehat{P}$ in $\widehat{G} / \widehat{H}$ is a section of the fibration, and the natural map $\widehat{L} \times \mathcal{F} \rightarrow \widehat{G} / \widehat{H}$ factors through a $\widehat{L}$ equivariant isomorphism of the product of $Z=\widehat{L} / \widehat{P}$ and the base $\widehat{G} / \widehat{H} \widehat{L}$. Since the product $\widehat{G}_{1}$ of the remaining factors of $\widehat{S}$ and the complex torus $\widehat{T}$ centralize $\widehat{L}$, this product stabilizes the fixed point set $\mathcal{F}$ and the manifold $\widehat{G} / \widehat{H}=Z \times \mathcal{F}=\widehat{L} \cdot x_{0} \times \widehat{G}_{1} \cdot x_{0}$ splits at the level of the groups as well.

Now let $G_{1}$ be the normal totally real subgroup of $G$ which is defined as the product of $T$ with the factors of $S$ which are not in $L$. In summary one should note that we have shown a "Borel-Remmert" type structure result $[\mathrm{BR}$, as was mentioned in the Introduction. 
Theorem 4.2. The CR-homogeneous space $M$ is the Cauchy-Riemann product of the compact complex flag manifold $Z=L . x_{0}$ and the CR-homogeneous space

$$
M_{1}=G_{1} \cdot x_{0}=G_{1} / H_{1} \hookrightarrow \widehat{G}_{1} / \widehat{H}_{1} .
$$

\section{Projective homogeneous spaces of codimen- sion at most two}

Here we continue in the setting of the previous section with $\widehat{G}$ a connected complex Lie group acting via a representation on a complex projective space $\mathbb{P}(V)$ with a real (connected) subgroup $G$ so that the orbit $G \cdot x_{0}=: M$ is the compact CR-homogeneous space of interest. The structure theorems allow us to assume that $\widehat{G}=\widehat{T} \widehat{S}$ is reductive with radical $\widehat{T}$ and semisimple part $\widehat{S}$ and that $G=T S$ is a real form. Since $G$ is a real algebraic group acting algebraically and $M$ is compact, we know that every maximal compact subgroup $K$ of $G$ acts transitively on $M$. Our goal here is to give a detailed description of this situation under the further assumption that $M$ is at most 2-codimensional as a $\mathrm{CR}$-manifold, i.e., at most 2-codimensional in the complex orbit $\widehat{G} \cdot x_{0}=\widehat{G} / \widehat{H}$.

\subsection{Description of the projective globalization}

The complex homogeneous manifold $X:=\widehat{G} \cdot x_{0}=\widehat{G} / \widehat{H}$ is the $\widehat{G}$-globalization in projective space of the CR-manifold $M=G \cdot x_{0}$ which is assumed to be of codimension at most two. Our goal here is to describe $X$ using now classical methods from the theory of actions of complex algebraic groups.

\subsubsection{The spherical property}

As a first step we show here that $X$ is spherical. This notion, which orginated in classical harmonic analysis, is naturally translated into the setting of actions of complex reductive groups to the condition that a Borel subgroup $\widehat{B}$ of $\widehat{G}$ has an open orbit in $X$, see $[\mathrm{VK}]$. By definition a Borel subgroup is a maximal, connected solvable subgroup of $\widehat{G}$. Such are complex algebraic subgroups and any two are conjugate (see e.g. [Bor] for the basic theory). Therefore the condition spherical is defined independent of the Borel sub- 
group in question. This condition turns out to be quite restrictive and leads to fine classification results which are of particular use in our situation.

There are a number of different ways to verify the spherical property. Here we focus on the $K$-action and use the Hamiltonian viewpoint. For this we let $\omega$ be a $K$-invariant Kählerian structure on $X$. Since we may assume that $K$ is represented as a group of unitary transformations, we may take this to be the restriction of the Fubini-Study form. In particular we have the associated $K$-equivariant moment map $\mu: X \rightarrow \mathfrak{k}^{*}$. Let $\widehat{K}$ denote the (reductive) complexification of $K$ in $\widehat{G}$.

Proposition 5.1. The manifold $X$ is $\widehat{K}$-spherical.

Proof. It is sufficient to show that the $K$-action on $X$ is coisotropic, i.e., that (generically) $\mu$-fibers are contained in the $K$-orbits $([\mathrm{HW}])$. For this we consider a dimension theoretically generic $K$-orbit $Y:=K . x$, which we know to be of codimension at most two. The tangent space of the $\mu$-fiber at $x$ is $T_{x} Y^{\perp_{\omega}}$, see, e.g., (26.3) in [GS]. We must show that this tangent space is contained in $T_{x} Y$.

Now the complex tangent space $T_{x}^{C R} Y$ to $Y$ in $T_{x} Y$ is a complex subspace of $T_{x} X$ of codimension at most two. Since $\omega$ is Kählerian, $T_{x} Y^{\perp_{\omega}}$ is contained in the orthogonal complement $P_{x}$ of $T_{x}^{C R} Y$ with respect to the induced Hermitian metric. Note that $P_{x}$ has a natural real structure with $P_{x}^{\mathbb{R}}$ being defined as $P_{x} \cap T_{x} Y$. Since $\widehat{K}$ acts transitively on $X$, the orbit $Y$ is not complex. So there are two cases to consider. First, if $P_{x}$ is complex 1-dimensional, i.e., $\operatorname{dim}_{\mathbb{R}} P_{x}^{\mathbb{R}}=1$, then $\operatorname{codim}_{\mathbb{R}}(Y)=1$ and $Y$ is odd-dimensional. But $\mu(Y)$ is a flag manifold and thus is even-dimensional; so the fiber of $\mu \mid Y$ is positivedimensional. Since the tangent space of the $\mu$-fiber at $x$ is $T_{x} Y^{\perp_{\omega}}$, the full $\mu$-fiber is 1-dimensional. Therefore it must be (locally) contained in $Y$, i.e., $Y$ is coisotropic.

In the case where $P_{x}$ is 2-dimensional, it follows that $\operatorname{codim}_{\mathbb{R}}(Y)=2$ and $P_{x}^{\mathbb{R}}$ is 2-dimensional. It would be theoretically possible that $K_{x}$ acts with positive dimensional orbits in $P_{x}$. But we have chosen $Y$ to be a generic $K$-orbit; so all nearby $K$-orbits are also $2-$ codimensional. As a result the connected component $K_{x}^{0}$ acts trivially on the 2 -dimensional complement $P_{x}^{\mathbb{R}}$ to $T_{x}^{C R} Y$ in $T_{x} Y$. Consequently, the orbit $N\left(K_{x}^{0}\right) \cdot x$ of the normalizer of $K_{x}^{0}$ in $K$ is at least 2 -dimensional. Now since $\mu(Y)$ is even dimensional, we know that 
either $\mu \mid Y$ has two dimensional fibers, in which case the result follows, or $\mu$ maps $Y$ bijectively onto a coadjoint orbit. In the latter case, in particular due to the fact that coadjoint orbits are simply-connected, one would have $K_{\mu(x)}=K_{x}^{0}$. But since coadjoint orbits are flag manifolds, $N\left(K_{\mu(x)}\right) \cdot x$ is finite and this is a contradiction. Thus $\mu \mid Y$ has 2-dimensional fibers and it follows that $Y$ is coisotropic.

\subsubsection{Affine-Rational fibrations}

As a result of Proposition 5.1 we now know that $X=\widehat{G} / \widehat{H}$ is $\widehat{G}$-spherical. Since $K$ acts transitively on $M$, its generic orbits in $X$ are at most 2codimensional. Letting $\widehat{G}_{u}$ to be a maximal compact subgroup of $\widehat{G}$ which contains $K$, the same is true of it. One therefore says that $X$ is a $\widehat{G}^{-}$ homogeneous spherical variety of rank at most two. A great deal is known about spherical varieties (see e.g. [Ak1], [Ak2], [BLV], [Bri], [LV]) so that it would be possible to give a detailed list of the manifolds $X$ which occur in our setting. Our goal here is to give sufficient detail so that for any given application the reader can work out whatever fine point is needed.

The "affine-rational" fibration, which is in a certain sense canonical, is the first method which we apply. As the name indicates, the basic building blocks of this fibration $\widehat{G} / \widehat{H} \rightarrow \widehat{G} / \widehat{Q}$ are affine and projective rational homogeneous varieties, the fiber being affine and the base being rational. This condition for the base is equivalent to $\widehat{Q}$ containing a Borel subgroup, i.e., the group $\widehat{Q}$ is (complex) parabolic. Using the most elementary aspects of root theory, the parabolic subgroups of $\widehat{G}$ can be described in complete detail (see, e.g., Bor]).

Since the possibility of having a nontrivial finite group $\widehat{H} / \widehat{H}^{0}$ causes only notational difficulties, in our discussion here we assume that $\widehat{H}$ is connected. Instead of applying the Levi-Malcev decomposition which is applicable in complete generality, we make use of the Levi-decomposition $\widehat{H}=\widehat{H}_{u} \rtimes \widehat{H}_{r}$ which is only valid for algebraic groups. Here $\widehat{H}_{u}$ is the unipotent radical of $\widehat{H}$ which consists of the unipotent elements of its radical, and $\widehat{H}_{r}$ is a maximal reductive subgroup. At this point the "hat notation" only indicates that we are dealing with complex algebraic groups and has nothing particular to do with the real group $G$. 
If $\widehat{H}$ is not reductive, then using what we call the Weisfeiler method (see Hum §30) there is a systematic constructive method for determining a parabolic group $\widehat{Q}=\widehat{Q}_{u} \rtimes \widehat{Q}_{r}$ with $\widehat{H}_{u} \subset \widehat{Q}_{u}$ and $\widehat{H}_{r} \subset \widehat{Q}_{r}$. The fibration $\widehat{G} / \widehat{H} \rightarrow \widehat{G} / \widehat{Q}$ has a projective rational manifold as its base. The fiber $\widehat{Q} / \widehat{H}$ is itself a homogeneous fiber bundle

$$
A:=\widehat{Q} / \widehat{H} \rightarrow \widehat{Q} / \widehat{Q}_{u} \widehat{H}=\widehat{Q}_{r} / \widehat{H}_{r}
$$

with fiber the affine homogeneous space $\widehat{Q}_{u} / \widehat{H}_{u}=\mathbb{C}^{n}$ and base which is the quotient of a reductive group by a reductive subgroup. The latter is affine algebraic and, since we are dealing with algebraic homogeneous bundles, it follows that the total space is also affine. Thus we refer to $\widehat{G} / \widehat{H} \rightarrow \widehat{G} / \widehat{Q}$ as an affine-rational fibration. It is in general not unique, but we force it a bit in the direction of unicity by assuming that the fiber is minimal in the sense that it can not be $\widehat{Q}$-equivariantly fibered over a projective rational manifold.

\subsubsection{The fiber of an affine-rational fibration}

In our case of interest where $X$ is at most of rank two, although the group $\widehat{Q}$ may not be acting as a reductive group on the fiber $A$, since the generic orbits of its maximal compact subgroups, e.g., $U:=\widehat{G}_{u} \cap \widehat{Q}$, are at most 2-codimensional, we view $A$ as a spherical variety of rank at most two in a slightly more general sense. We now describe all possible cases which can occur for $A=\widehat{Q} / \widehat{H}$ (see the table below). Our detail is sufficient so that by using elementary root and representation theory precise combinatorial descriptions can be determined.

Recall that the center of $\widehat{Q}$ is a complex torus $\widehat{T} \cong\left(\mathbb{C}^{*}\right)^{r}$ and consider the fibration $\widehat{Q} / \widehat{H} \rightarrow \widehat{Q} / \widehat{T} \widehat{H}$. The fiber is $\left(\mathbb{C}^{*}\right)^{n}$. Since the base is affine and for each $\mathbb{C}^{*}$ the codimension of the generic $U$-orbit in the base decreases by one, it follows that $0 \leq n \leq 2$. If $n=2$, it follows that $\widehat{H}_{u}=\widehat{Q}_{u}$. In that case the semisimple parts $\widehat{H}_{r}^{s s}$ and $\widehat{Q}_{r}^{s s}$ also agree and in fact $\widehat{Q}$ is just acting as $\left(\mathbb{C}^{*}\right)^{2}$. In other words, $\widehat{G} / \widehat{H} \rightarrow \widehat{G} / \widehat{Q}$ is a $\left(\mathbb{C}^{*}\right)^{2}$-principal bundle.

If $n=1$, then the generic $U$-orbits in $\widehat{Q} / \widehat{T} \widehat{H}$ are 1 -codimensional. This is a situation which is described in detail in ([AHR $]$. There are two cases, i.e., either $\widehat{Q}_{u}$-acts or it doesn't. If it does, then, again since the codimension of 
the $U$ goes down every time one fibers by a noncompact fiber, it follows that $\widehat{Q}_{u}$ acts transitively on $\widehat{Q} / \widehat{T} \widehat{H}=\mathbb{C}^{m}$ and $U$ is acting linearly there as either the compact symplectic group, $\mathrm{SU}_{m}$ or $\mathrm{U}_{m}$. If $\widehat{Q}_{u}$ acts trivially on this manifold, i.e., if $\widehat{Q}_{u}=\widehat{H}_{u}$, then it is just the quotient $\widehat{Q}_{r}^{s s} / \widehat{H}_{r}^{s s}$ of the semisimple parts of the Levi-factors. Since the compact group $U$ has 1-codimensional orbits, we know that this manifold is just a semisimple symmetric space of rank 1, i.e., the tangent bundle of either the sphere $S^{n}$ or its $2: 1$ quotient $\mathbb{P}_{2}(\mathbb{R})$, complex projective space $\mathbb{P}_{n}(\mathbb{C})$, hyperbolic projective space $\mathbb{P}_{n}(\mathbb{H})$ or the Cayley plane $\mathbb{P}_{2}(\mathbb{O})$. These last results are due to Morimoto and Nagano $([\mathrm{MN}])$.

Up to this point we have only handled the case where the $\widehat{T}$-action on $A$ is nontrivial. If it is trivial, then we consider the fibration $\widehat{Q} / \widehat{H} \rightarrow \widehat{Q} / \widehat{Q}_{u} \widehat{H}$ which has $\mathbb{C}^{n}$ as its fiber. As usual there are two cases: The base is either nontrivial or it isn't! If it is trivial, this means that the reductive parts $\widehat{Q}_{r}$ and $\widehat{H}_{r}$ are the same and that $\widehat{H}$ is constructed from $\widehat{Q}$ by removing two root groups. This is a situation that is easily classified. If the base $\widehat{Q} / \widehat{Q}_{u} \widehat{H}$ is nontrivial, then, since the center of $\widehat{Q}$ acts trivially on $A$, this is again the quotient of the semisimple parts of $\widehat{Q}_{r}$ and $\widehat{H}_{r}$, i.e., a complex semisimple symmetric space of rank one as above. Once one understands all of the possibilities for the pairs $(\widehat{Q}, \widehat{H})$ with this property, one only needs to sort out those where a root group of $\widehat{Q}_{u}$ can be removed. This type of combinatorial discussion has also been carried out in ([AHR]).

Finally we come to the case where both the center $\widehat{T}$ and the unipotent radical $\widehat{Q}_{u}$ both act trivially on the fiber $A$. In other words, after moding out ineffectivity $A$ is a semisimple affine spherical space of rank two. These have been classified by M.Krämer $([\mathrm{Kr}])$ with very useful additional remarks being given by D. Akhiezer $([\mathrm{Ak} 2])$. Here is one way of thinking about this classification.

With one exception where $\widehat{Q}$ is acting as $\mathrm{SO}_{9}$ with isotropy $\mathrm{Spin}_{7}$, all examples occur either as affine symmetric spaces of rank two or are naturally defined bundles over symmetric spaces of rank one which were described as above. The two infinite series of bundles are the following:

$$
\mathrm{SL}_{m+1} / S L_{m} \rightarrow \mathrm{SL}_{m+1} / \mathrm{SL}_{m} \cdot \mathbb{C}^{*}
$$

and

$$
\mathrm{Sp}_{2 n} /\left(\mathrm{Sp}_{2 n-2} \times \mathbb{C}^{*}\right) \rightarrow \mathrm{Sp}_{2 n} / \mathrm{Sp}_{2 n-2} \times \mathrm{Sp}_{2}
$$


Let us describe these examples in further detail. In the case of (13) the base is the tangent bundle of $\mathbb{P}_{m}$ and the fiber is $\mathbb{C}^{*}$. The total space can be regarded as the tangent bundle of the total space of the unit circle bundle of the hyperplane bundle over $\mathbb{P}_{m}$. In the case of (4) the base is the tangent bundle of $\mathbb{P}_{n}(\mathbb{H})$ and the fiber is the 2-dimensional affine quadric. For this latter point it is important to recall that $\mathrm{Sp}_{2}=\mathrm{SL}_{2}$. Note that this manifold is the tangent bundle of a very natural $S^{2}$-bundle over $\mathbb{P}_{n}(\mathbb{H})$.

The above descriptions of the fiber $A$ of the affine-rational fibration $\widehat{G} / \widehat{H} \rightarrow$ $\widehat{G} / \widehat{Q}$ are summarized in the following table. Note that the classification of symmetric spaces can be found in Helg.

\begin{tabular}{|c|c|c|}
\hline fiber & base & remarks \\
\hline \multicolumn{3}{|c|}{$\widehat{T}$-fibration } \\
\hline$\left(\mathbb{C}^{*}\right)^{2}$ & point & $\widehat{G} / \widehat{H} \stackrel{\left(\mathbb{C}^{*}\right)^{2}}{\rightarrow} \widehat{G} / \widehat{Q}$ is principal \\
\hline $\mathbb{C}^{*}$ & $\mathbb{C}^{m}$ & linear $U$-action on $\widehat{Q} / \widehat{T} \widehat{H}$ \\
\hline \multicolumn{3}{|c|}{$\widehat{Q}_{u}$-fibration } \\
\hline $\mathbb{C}^{n}$ & point & $\widehat{H}=\widehat{Q}-2$ root groups \\
\hline $\mathbb{C}^{n}$ & symmetric space of rank 1 & $\widehat{H}_{u}=\widehat{Q}_{u}-1$ root group \\
\hline $\mathbb{C}^{*}$ & $T\left(\mathbb{P}_{m}\right)$ & $A=\mathrm{SL}_{m+1}(\mathbb{C}) / \mathrm{SL}_{m}(\mathbb{C})$ \\
\hline $\mathrm{SL}_{2}(\mathbb{C}) / \mathbb{C}^{*}$ & $T\left(\mathbb{P}_{n}(\mathbb{H})\right)$ & $A=\operatorname{Sp}_{2 n}(\mathbb{C}) /\left(\operatorname{Sp}_{2 n-2}(\mathbb{C}) \times \mathbb{C}^{*}\right)$ \\
\hline \multicolumn{3}{|c|}{ affine spherical spaces of rank two } \\
\hline \multicolumn{3}{|c|}{ one exceptional case: $\mathrm{SO}_{9}(\mathbb{C}) / \operatorname{Spin}_{7}(\mathbb{C})$} \\
\hline
\end{tabular}

Table 1: Fiber $A=\widehat{Q} / \widehat{H}$ of the affine-rational fibration

\subsection{The case of a nontrivial radical}

Here we return to the study of the $G$-action on the $\widehat{G}$-homogeneous space $\widehat{G} / \widehat{H}$. As always in the section we assume that $M=G \cdot x_{0}$ is at most 2 codimensional. Here we describe how to handle the situation where the radical $R$ of $G$ is nontrivial. Of course we do this by a case-by-case analysis 
of the sequence of fibrations

$$
\widehat{G} / \widehat{H} \rightarrow \widehat{G} / \widehat{R} \widehat{H} \rightarrow \widehat{G} / \widehat{Q}
$$

The fiber is $\left(\mathbb{C}^{*}\right)^{n}$, and, assuming that $M$ is at most 2 -codimensional and that $R$ is nontrivial, one has $n=1,2$. Note that in the case where $n=2$ the group $G$ acts transitively on the base $\widehat{G} / \widehat{Q}$. If $n=1$, there are two cases: either $\widehat{R} \widehat{H}=\widehat{Q}$, and $M$ is mapped to a real hypersurface orbit of $G$ in the

base, or $G$ acts transitively on $\widehat{G} / \widehat{Q}$ and $M$ is mapped to a $G$-hypersurface orbit in the noncompact manifold $\widehat{G} / \widehat{R} \widehat{H}$.

In the case where $n=2$ the manifold $M$ is just $\left(S^{1}\right)^{2}$-principal bundle over the compact base $\widehat{G} / \widehat{H}$ where $G$ is acting transitively. If $n=1$ and $G$ is again acting transitively on the base, then $M$ is just an $S^{1}$-principal bundle over the hypersurface orbit of the semisimple part of $G$ in $\widehat{G} / \widehat{R} \widehat{H}$. A precise description of this hypersurface setting can be found in ([AHR]). The remaining situation is where $n=1$ and $\widehat{G} / \widehat{R} \widehat{H}=\widehat{G} / \widehat{Q}$ and $M$ is mapped to a $G$-hypersurface orbit in the base. Below we give a complete description in the base and then elementary considerations show which $S^{1}$-principal bundles arise over the $G$-hypersurface orbit.

Thus, except for this last case which is handled below, the existence of a nontrivial radical allows us to reduce the classification to known results.

\subsection{Transitive action on the base}

The following is a major simplifying step for our description of the situations where $G$ is noncompact.

Theorem 5.1. If $X=\widehat{G} / \widehat{H}$ is noncompact and the real form $G$ acts transitively on the base $Y=\widehat{G} / \widehat{Q}$ of an affine-rational fibration, then $G$ is compact.

The proof will be given in detail for the case where $G$ is semisimple, which we now assume, and at the end we will note the necessary adjustments to handle the case where $G$ has a nontrivial radical. We require several intermediate steps where it is assumed to the contrary that $G$ does act transitively on the base of the given affine-rational fibration. Let us begin by recalling that, although $G$ is a real form of $\widehat{G}$ in its representation in the projective 
linear group, it is possible that this is not the case for its action on $Y$. Fortunately, this can happen in only one way, namely when one or more simple factors $S$ of $G$ are themselves complex Lie groups which are embedded as a real form of their complexifications $S \times S$ as antiholomorphic diagonals. This means that there is an antiholomorphic automorphism $\varphi: S \rightarrow S$ with $S$ embedded by $s \mapsto(s, \varphi(s))$. If either factor of the complexification $S \times S$ acts trivially on $Y$, then the group $S$ which was originally a real form acts holomorphically as a complex Lie group.

Now $Y$ splits into a product of homogeneous rational factors of the simple factors of $\widehat{G}$ (modulo ineffectivity). We write it as

$$
Y=\Pi_{i} Y_{i}^{1} \times \Pi_{j} Y_{j}^{2,1} \times \Pi_{k} Y_{k}^{2,2}
$$

where the simple noncomplex factor $S_{i}$ acts transitively on $Y_{i}^{1}$ as a real form of its simple complexification $\widehat{S}_{i}$, the simple complex factor $S_{j}$ acts as a real form of its complexification $S_{j} \times S_{j}$ on $Y_{j}^{2,1}$ where the latter acts almost effectively, and the simple complex factor $S_{k}$ acts as a complex Lie group on $Y_{k}^{2,2}$ where its complexification also acts as $S_{k}$. We will immediately see that most of these possibilities can not occur if $G$ is to act transitively on $Y$.

For example, if we write $Y_{j}^{2,1}$ as $S / Q_{1} \times S / Q_{2}$, then in order for the diagonally embedded copy of $S$ to act transitively on this product, we must have a situation where a parabolic subgroup $Q_{2}$ of $S$ would necessarily act transitively on the rational manifold $S / Q_{1}$. Taking conjugates so that $Q_{1}$ and $Q_{2}$ contain the same Borel subgroup, elementary root considerations show that this is not possible. Hence, factors of the type $Y_{j}^{2,2}$ don't occur.

Our strategy for proving Theorem 5.1 is to first handle the case where $G$ and $\widehat{G}$ are simple (Proposition 5.2), then the case where $G$ is simple and complex with its complexification being $\widehat{G}=G \times G$ (Proposition 5.4), and finally to put things together in Theorem 5.1.

\subsubsection{Transitive action of a simple real form}

Proposition 5.2. If $\widehat{G}$ is simple, $\widehat{Q}$ is a proper subgroup of $\widehat{G}$ and $G$ acts transitively on $\widehat{G} / \widehat{Q}$, then $G$ is compact.

The proof requires a bit of preparation. First, the classification work of A. Onishchik ([01, O2]) shows that there are only two series of examples 
where the situation in the proposition could occur, i.e., where a simple real form $G$ acts transitively on a homogeneous rational manifold $Y=\widehat{G} / \widehat{Q}$ of its simple complexification. They can be described as follows:

1. The odd dimensional complex projective space $\mathbb{P}_{2 n-1}$

2. The space $\mathcal{C}_{n}$ of complex structures on $\mathbb{R}^{2 n}$.

These are compact Hermitian symmetric spaces which have respective isometry groups $\mathrm{SU}_{2 n}$ and $\mathrm{SO}_{2 n}(\mathbb{R})$. As coset spaces they are then described as $\mathbb{P}_{2 n}=\mathrm{SU}_{2 n} / \mathrm{U}(2 n-1)$ and $\mathcal{C}_{n}=\mathrm{SO}_{2 n}(\mathbb{R}) / \mathrm{U}(n)$. In the first case the real form of $\mathrm{SL}_{2 n}(\mathbb{C})$ which acts transitvely is the group of quaternionic linear transformations $\mathrm{SL}_{2 n}(\mathbb{H})$ and of course its maximal compact subgroup $\mathrm{USp}_{2 n}$ acts transitively as well. In the second case the noncompact real form is $\mathrm{SO}(1,2 n-1)$ with maximal compact subgroup $\mathrm{SO}_{\mathbb{R}}(2 n-1)$ also acting transitively on $\mathcal{C}_{n}$.

To prove Proposition 5.2 amounts to showing that none of these cases can occur in our situation. One of the situations which arises (and must be eliminated) is where the unipotent radical $\widehat{Q}_{u}$ acts trivially on the fiber $\widehat{Q} / \widehat{H}$. Before coming to the proof of Proposition 5.2, we note a fact that is useful in handling that case.

Proposition 5.3. Let $\widehat{G}=K_{G}^{\mathbb{C}}$ be a complex reductive group with reductive subgroups $\widehat{H}$ and $\widehat{L}$ with the property that $\widehat{L}=K_{L}^{\mathbb{C}}$ has an open orbit $\Omega$ in the affine homogeneous space $\widehat{G} / \widehat{H}$. Assume that the maximal compact group $K_{G}$ contains $K_{L}$ and that all groups under consideration are connected. Then the (dimension theoretically) minimal $K_{G}$-orbits in $\widehat{G} / \widehat{H}$ are contained in $\Omega$ and are $K_{L}$-orbits. In particular, $\Omega$ is also affine.

Proof. The real dimension of a dimension theoretically minimal $K_{G}$-orbit $N$ agrees with the complex dimension of $\widehat{G} / \widehat{H}$, i.e., that of $\Omega$. All $K_{L}-$ orbits in $\Omega$ have at least this dimension. Thus, if a $K_{G}$-orbit has nonempty intersection with $\Omega$ and is minimial, then it is contained in $\Omega$. On the other hand, no minimal $K_{G}$-orbit is contained in a proper complex analytic subset of $\widehat{G} / \widehat{H}$, because it totally real with its real dimension being the same as the complex dimension of $\widehat{G} / \widehat{H}$. Applying this to the complement of $\Omega$, the desired result follows. 
Example. It should be pointed out that in the above setting $\widehat{L}$ doesn't necessarily act transitively on $\widehat{G} / \widehat{H}$. For example, if $\widehat{G}=\mathrm{SL}_{3}(\mathbb{C})$ and $\widehat{H} \cong \mathrm{GL}_{2}(\mathbb{C})$ is the stabilizer in $\widehat{G}$ of the decomposition $\mathbb{C}^{3}=\operatorname{Span}\left(e_{1}\right) \oplus \operatorname{Span}\left(e_{2}, e_{3}\right)$, then in fact $\widehat{H}$ has an open orbit in $\widehat{G} / \widehat{H}$.

Suppose that the unipotent radical $\widehat{Q}_{u}$ acts trivially on the fiber $\widehat{Q} / \widehat{H}$. Then, making an intermediate fibration if necessary, we may assume that this fiber is either $\left(\mathbb{C}^{*}\right)^{n}$ or an affine symmetric space of the group $\widehat{Q}_{r}$. Since the center of $\widehat{Q}_{r}$ is 1 -dimensional in both cases, if the first case occurs, then $n=1$. Thus, to prove Proposition 5.2 we must eliminate the following three cases: 1.) The unipotent radical $\widehat{Q}_{u}$ acts transitively on $\widehat{Q} / \widehat{H}, 2$.) $\widehat{Q} / \widehat{H}=\mathbb{C}^{*}$, and 3.) The radical of $\widehat{Q}_{r}$ acts trivially on $\widehat{Q} / \widehat{H}$ and with $\widehat{Q} / \widehat{H}$ being a symmetric space of the semisimple part $Q_{r}^{s s}$.

Proof of Proposition 5.2 in cases 1.) and 2.): Note that the representation of $\widehat{Q}_{r}$ on $\widehat{Q}_{u}$ is irreducible and $\widehat{Q}_{u}$ can be identified with the tangent space of the Hermitian symmetric space $\widehat{G} / \widehat{Q}$. Thus, if $\widehat{Q}_{u}$ acts transitively on the fiber, then $\widehat{G} / \widehat{H}$ is just the tangent bundle of $\widehat{G} / \widehat{Q}$. Now in both cases $\widehat{Q}_{r}$ is some $\mathrm{GL}_{m}(\mathbb{C})$ whose center acts as scalar multiplication by $\mathbb{C}^{*}$ on the tangent space at the neutral point. As usual denote the basic character by Det $: \widehat{Q}_{r} \rightarrow \mathbb{C}^{*}$. Now, using the standard choices of a Cartan decomposition $\mathfrak{g}=\mathfrak{k} \oplus \mathfrak{p}$ and a maximal Abelian subspace $\mathfrak{a}$ of $\mathfrak{p}$, one checks that the isotropy subgroup of the $G$-action on the base contains a Cartan subgroup $H=T \times A$ with $\operatorname{Det}(A)=\mathbb{R}^{>0}$. Thus $A$ can not stabilize a compact subset of the tangent space and therefore this case doesn't occur. Case 2.) goes in the same way, because the $\mathbb{C}^{*}$-bundle is defined by some power Det $^{k}$.

Proof of Proposition 5.2 in case 3.): Here we just note that the fiber $\widehat{Q} / \widehat{H}$ is an affine symmetric space of some $\mathrm{SL}_{m}(\mathbb{C})$. If it is of rank one, then it is the tangent bundle of a projective space and the same argument as above shows that the $G$-orbit can not be compact. If it is rank two, then at the level of compact groups it is either a Grassmannian of 2 -planes $\mathrm{SU}(2+q) / S(\mathrm{U}(2) \times$ $\mathrm{U}(q))$ or one of the isolated cases $\mathrm{SU}(3) / \mathrm{SO}(3)$ or $\mathrm{SU}(6) / \mathrm{USp}(6)$. Here we apply Proposition 5.3: $K . x_{0}=M$ fibers over $\widehat{G} / \widehat{Q}=K / L$, where $L . x_{0}$ is 2-codimensional (over $\mathbb{R}$ ) in $\widehat{Q} / \widehat{H}$. Since $L . x_{0}$ can not be complex (it would then be a compact complex submanifold of an affine manifold), the orbit $\widehat{L} \cdot x_{0}$ of its complexification is open in the fiber. Thus Proposition 5.3 implies that 
the smaller group $L$ also acts transitively on the compact symmetric space of rank 2. It is known that no smaller compact group acts transtively on any Grassmannian except for projective space ([01, O2]).

A direct check of the groups in our cases shows that $\mathrm{SU}(3) / \mathrm{SO}(3)$ does not occur. Finally, SU(5) does indeed act transitively on $\mathrm{SU}(6) / \mathrm{USp}(6)=$ $\mathrm{SU}(5) / \mathrm{USp}(4)$. However, it is not a symmetric space and does not fiber over a symmetric space and is therefore not spherical of rank two!

\subsubsection{Simple real forms which are complex}

The result in this case can be stated as follows.

Proposition 5.4. The group $G$ is not a simple complex group which is a real form of $\widehat{G}=G \times G$ and which acts transitively on a positive-dimensional base $\widehat{G} / \widehat{Q}$.

Proof. As in the previous case, the proof requires a bit of background. First, we consider the decomposition $G=K A N$. Since $G$ is complex, $N$ is the unipotent radical of a complex Borel subgroup and $A \cong\left(\mathbb{R}^{>0}\right)^{r}$ is a noncompact real form of a maximal complex torus. Since $M$ is compact and $A N$ is acting algebraically, it follows that it has a fixed point in $M$. Therefore we may assume that the isotropy subgroup $\widehat{H}$ contains its complexification $\widehat{A N}=T \ltimes(N \times N)$ in $\widehat{G}$. Here $T \cong\left(\mathbb{C}^{*}\right)^{r}$ is the maximal complex torus mentioned above which is (holomorphically) diagonally embedded in $\widehat{G}=G \times G$.

To keep things straight we refer to the first factor of the complexification as $G_{1}$ and the second as $G_{2}$ and consider the sequence of fibrations

$$
G_{1} \times G_{2} /(T \ltimes(N \times N)) \rightarrow G_{1} \times G_{2} / H \rightarrow G_{1} \times G_{2} /\left(H_{1} \times H_{2}\right) .
$$

Here $H_{1}$ and $H_{2}$ are the respective projections of $H$ into the factors $G_{1}$ and $G_{2}$. Since they contain the Borel group $T \ltimes N$, they are parabolic and these quotients are compact. By assumption $G$ acts transitively on the base $\widehat{G} / \widehat{Q}$. Thus $\widehat{Q}$ contains one of the factors, say $G_{2}$, and it follows that $\widehat{Q}=H_{1} \times G_{2}$. So the minimality assumption, i.e., that the fiber can not be nontrivially fibered onto a positive-dimensional compact base, implies that $H_{2}=G_{2}$. However, this implies that a maximal semisimple subgroup $H_{r}^{s s}$ contains $G_{2}$ as a simple factor and by our effectivity assumption it therefore follows that 
$H$ contains a diagonally embedded copy of $G$. Since such is a maximal subgroup and $(G \times G) / G$ is affine, this violates our assumption that the base is positive-dimensional.

As we observed above, if $G$ is simple, complex and embedded via an antiholomorphic automorphism as a real form of $G \times G$, then $G$ can not operate transitively on the product $G / P_{1} \times G / P_{2}$ of $G$-homogeneous rational manifolds. However, interesting $2-$ codimensional orbits can arise in this way.

Example. Let $V$ be a complex vector space and equip $V \oplus V^{*}$ with its standard symmetric complex bilinear form $b$ which is defined by $b(v, f)=$ $f(v)$ and is invariant by the diagonal action of $G=\operatorname{SL}(V)$. This defines a $G$-invariant, complex hypersurface

$$
D=\left\{([v],[f]) \in \mathbb{P}(V) \times \mathbb{P}\left(V^{*}\right) ; b(v, f)=0\right\} .
$$

In fact, $G$ acts transitively on both $D$ and its complement in $\mathbb{P}(V) \times \mathbb{P}\left(V^{*}\right)$, the latter being the rank one affine symmetric space which is the cotangent bundle of $\mathbb{P}(V)$.

Choosing a basis of $V$ and letting $z$ and $w$ be the associated coordinates of $V$ and $V^{*}$, one writes $b(z, w)=z^{t} w$. At the matrix level the $G$-action is given by $z \mapsto A z$ and $w \mapsto\left(A^{t}\right)^{-1} w$. One can view this as an action on $\mathbb{P}_{n} \times \mathbb{P}_{n}$ defined by the holomorphic automorphism $A \mapsto\left(A^{t}\right)^{-1}$. Changing this slightly, we consider the action given by $(z, w) \mapsto(A z, \varphi(A) w)$, where $\varphi(A)=\left(\bar{A}^{t}\right)^{-1}=$ $A^{\dagger}$. In this way $\mathbb{P}_{n} \times \mathbb{P}_{n}$ is a two orbit variety with the lower-dimensional orbit being the real 2-codimension manifold $M:=\left\{([z],[w]) ; z^{t} \bar{w}=0\right\}$.

The abstract setup of this example is the following. Let $S$ be a complex Lie group equipped with an antiholomorphic automorphism $\varphi: S \rightarrow S$. If $I$ is a closed complex subgroup, then so is $\varphi(I)$. The mapping $\varphi$ induces an antiholomorphic $S$-equivariant diffeomorphism $S / I \rightarrow S / \varphi(I)$ where the action on the image space is given by $t(s I):=\varphi(t) s I$. If $I_{1}$ and $I_{2}$ are two closed complex subgroups of $S$, then the diagonal action on $S / I_{1} \times S / \varphi\left(I_{2}\right)$ of the real form $S$ of $S \times S$ defined by $s \mapsto(s, \varphi(s))$ is just the transfer of the standard holomorphic diagonal action on $S / I_{1} \times S / I_{2}$ by the identity on the first factor and this antiholomorphic map on the second. If $I_{1}=I_{2}=I$ and $Z=S / I$, then we write $Z \times \bar{Z}$ for the product $S / I \times S / \varphi(I)$ 
Proposition 5.5. Let $G$ be a simple complex Lie group which is a real form of $\widehat{G}=G \times G$ via an antiholomorphic automorphism $\varphi$. Suppose that $G$ has a closed orbit $M$ of codimension at most two in a product $Y=\widehat{G} / \widehat{Q}=$ $G / Q_{1} \times G / Q_{2}=Y_{1} \times Y_{2}$. Then $G=\operatorname{SL}(V), Y_{1}=Z=\mathbb{P}(V)=G / Q$, $Y_{2}=\bar{Z}=G / \varphi(Q)$ and $Y=Z \times \bar{Z}$. In coordinates the manifold $M$ is the transfer of the complex hypersurface $D$ in the above example by the map $Z \times Z \rightarrow Z \times \bar{Z}$ which is induced by $\varphi$.

Proof. We may write $Q_{2}$ as $\varphi\left(Q_{2}\right)$ and consider the standard (holomorphic) diagonal action of $G$ on $G / Q_{1} \times G / Q_{2}$. The only possibility for a closed orbit of real codimesion at most two is to have a complex hypersurface orbit $E$ in this setup. Since a maximal compact subgroup $K$ of $G$ acts transitively on $E$, one easily checks that the generic orbits of $K$ in the complement of $E$ are real hypersurfaces, e.g., because the normal bundle of $E$ can not be topologically trivial.

We regard the open $G$-orbit $Y \backslash E$ as a bundle over $G / Q_{1}$ with a fiber which is the open orbit of the parabolic group $Q_{1}$ in $G / Q_{2}$ and see that $Q_{2}$ is a maximal parabolic, because otherwise $G / Q_{2}$ would fiber over some $G / P$ where the parabolic $Q_{1}$ would necessarily act transitively which is impossible. Consequently, $\operatorname{Pic}\left(G / Q_{2}\right) \cong \mathbb{Z}$ and thus the lower-dimensional $Q_{1}$-orbit $E \cap$ $Y_{2}$ is an ample divisor and as a result its complement in $G / Q_{2}$ is affine.

From the classification of the homogeneous affine varieties where the maximal compact subgoup $L$ at hand has real hypersurfaces as its generic orbits (see AHR]) we know that the only case where there is a larger group than $L^{\mathbb{C}}$ acting is the case of $\mathbb{P}(V)$ where the group is the isotropy subgroup of the $\mathrm{SL}(V)$-action. Applying this argument to both factors we have the desired result.

\subsubsection{The case of several factors}

We now turn to the final step in the proof of Theorem 5.1, namely to handle the case where the group $G$ is semisimple but not simple. At the end we remark how to handle the case of a nontrivial radical.

Proof of Theorem 5.1: Let us review the situation. Here $G$ is a semisimple real form of $\widehat{G}$ and $M=G \cdot x_{0} \hookrightarrow \widehat{G} \cdot x_{0}=\widehat{G} / \widehat{H}$. We assume that the given 
affine-rational fibration $X=\widehat{G} / \widehat{H} \rightarrow \widehat{G} / \widehat{Q}$ has positive dimensional fiber and that the real form $G$ acts transitively on the base. Also recall that we may assume that $G$ is (up to connected components) the stabilizer of $M$ in $\widehat{G}$ and that the action of $G$ on $X$ is almost effective.

Under these conditions we show that $G$ is compact. For this we assume to the contrary that $G_{1}$ is a simple noncompact real form of $\widehat{G}_{1}$ with a complementary semisimple factor $G_{2}$ so that $Y=\widehat{G}_{1} / \widehat{Q}_{1} \times \widehat{G}_{2} / \widehat{Q}_{2}$. We project the base onto the the second factor $\widehat{G}_{2} / \widehat{Q}_{2}$ and consider the fiber $\widehat{G}_{1} \times \widehat{Q}_{2} / \widehat{H} \widehat{Q}_{2}$ of the associated fibration of $X$. Here we replace $M$ by its intersection with this fiber which is a $\mathrm{CR}$-homogeneous manifold with respect to $G_{1} \times\left(G_{2} \cap \widehat{Q}_{2}\right)$. Since this group acts transitively on the base $\widehat{G}_{1} / \widehat{Q}_{1}$, we are almost in a position to apply induction on dimension. However, the way we have set up our argument, i.e., assuming that $G$ is semisimple, the induction assumption does not apply. Thus we must consider the action of the radical $Z$ of $\left(G_{2} \cap \widehat{Q}_{2}\right)$. It acts as a compact, central subgroup and we consider the intermediate fibration

$$
\widehat{G}_{1} \times \widehat{Q}_{2} / \widehat{H} \rightarrow \widehat{G}_{1} \times \widehat{Q}_{2} / \widehat{H} \widehat{Z} .
$$

The fiber is $\left(\mathbb{C}^{*}\right)^{n}$ for $n=0,1,2$ with $M$ being fibered as an $\left(S^{1}\right)^{n}$-bundle.

If $n=2$, then the base is already $\widehat{G}_{1} / \widehat{Q}_{1}$ where the noncompact real form $G_{1}$ is assumed to act transitively. If $\widehat{G}_{1}$ is simple, then on the base we have one of the exceptional examples given to us by Onishchik ([O1, O2]). But, as we have already seen, every character on $\widehat{Q}_{1}$ restricts to a charactor on the $G_{1}$-isotropy which takes its values in $\mathbb{R}^{>0}$. Since such can not stabilize the compact fiber $\left(S^{1}\right)^{2}$, it follows that the $G_{1}$-isotropy acts trivially on the fiber. Thus the $G_{1}$-orbits in $M$ are sections and at the complex level the bundle is a topologically trivial $\left(\mathbb{C}^{*}\right)^{2}$-bundle over $\widehat{G}_{1} / \widehat{Q}_{1}$. For complex geometric reasons (the base is rational) or by a simple group argument, this implies that the bundle is holomorphically trivial and that the $\widehat{G}_{1}$-orbits are sections which then agree with the $G_{1}$-orbits. Thus $\widehat{G}_{1}$ stabilizes the original $M$ (not just the intersection with this fiber), contrary to our assumption that $G$ is a real form and agrees with the stabilizer of $M$ in $\widehat{G}$. Note that if $G_{1}$ is complex and $\widehat{G}_{1}=G_{1} \times G_{1}$, then it would be theoretically possible for $G_{1}$ to act transitively on the base. However, this would mean that one of the factors of $\widehat{G}_{1}$ would act trivially and would therefore act almost effectively 
on the fiber $\left(\mathbb{C}^{*}\right)^{2}$. This is of course not possible and therefore the argument for the case $n=2$ is complete.

If $n=1$, then we consider the base $\widehat{G}_{1} \times \widehat{Q}_{2} / \widehat{H} \widehat{Z}$ in more detail. Now here the unipotent radical of $\widehat{Q}_{2}$ could theoretically be acting nontrivially. On the other hand the relevant group $G_{2} \cap \widehat{Q}_{2}$ for the CR-fibration is of the form $Z \cdot S$ where $S$ is semisimple. So in the discussion we may replace $\widehat{Q}_{2}$ by $\widehat{Z} \widehat{S}$ so that the group acting on the base of this intermediate fibration is the semisimple group $\widehat{G}_{1} \times \widehat{S}$. By a similar argument to that above, $G_{1} \times S$ is acting here as a real form of $\widehat{G}_{1} \times \widehat{S}$ and the case where one of the simple real factors is complex can not occur. Now it is theoretically possible that some complex simple factor of $\widehat{G} \times S$ stablizes the image of $M$ here and therefore in order to have the induction assumption, we must factor it out, thereby splitting off a complex factor of $M$. If this factor is not $\widehat{G}_{1}$, then we apply induction to the resulting manifold to obtain the desired contradiction. If it is $\widehat{G}_{1}$, then we restrict the $\mathbb{C}^{*}$-bundle to the corresponding compact orbit of $\widehat{G}_{1}$. Due to our minimality assumption on the affine-rational fibration $\widehat{G} / \widehat{H} \rightarrow \widehat{G} / \widehat{Q}=Y$, this orbit is just the same as the $G_{1}$-orbit in $Y$, i.e., $\widehat{G}_{1} / \widehat{Q}_{1}$ where $G_{1}$ is also acting transitively. Thus we are now in a position to use the same arguments here as we did in the case above of the $\left(\mathbb{C}^{*}\right)^{2}$-bundle over $\widehat{G}_{1} / \widehat{Q}_{1}$ to complete the proof in this case.

Finally, if $n=0$, then we may replace $\left(G_{2} \cap \widehat{Q}_{2}\right)$ by a semisimple group $S$ so that we are dealing with a CR-manifold $M . x_{0}=G_{2} S . x_{0}$ of a semisimple group where the induction assumption may be applied.

In order to simplify the above discussion, we assumed that $G$ is semisimple. Inspection of the proof in the cases where the radical of $\widehat{Q}_{2}$ acts $(n=1,2$ above) shows that exactly the same arguments handle the case where the radical of $G$ is nontrivial. This completes the proof of Theorem 5.1.

\subsection{Actions of real forms on flag manifolds}

We now turn to the case where $G$ is not acting transitively on the base of an affine-rational fibration $\widehat{G} / \widehat{H} \rightarrow \widehat{G} / \widehat{Q}$ and for the moment only analyze the $G$-action on the homogeneous rational manifold $Y=\widehat{G} / \widehat{Q}$ where we assume that the $G$-orbit $G \cdot x_{0}=: M$ of interest is either $1-$ or $2-$ codimensional. Recall that $Y$ splits $Y=Y_{1} \times \cdots \times Y_{m}$ according to the splitting of $\widehat{G}=\widehat{G}_{1} \cdots \widehat{G}_{m}$ 
into its simple factors. Thus if one of the simple factors of $G$ happens to be acting as a complex simple factor of $\widehat{G}$ we split off the corresponding factor (say $Y_{1}$ ) of $Y$ which will then define a product structure $M=Y_{1} \times M_{1}$. Having done this we see that $M$ is a product of certain factors of $Y$ together with a $G$-orbit where $G$ is acting as a real form. Thus it is sufficient to consider the case where $G$ is a real form of $\widehat{G}$.

Furthermore, $Y$ splits into a product $Y=Y_{1} \times \cdots \times Y_{m}$ according the splitting of $\widehat{G}$ into factors $\widehat{G}_{i}$ which are the complexifications of the simple factors $G_{i}$ of $G$. Since $M$ splits accordingly, it is enough to study the actions of the $G_{i}$ on the $Y_{i}$. Note that if one of the $\widehat{G}_{i}$ is not simple, then $G_{i}$ is complex and embedded in $\widehat{G}_{i}=G_{i} \times G_{i}$ via an antiholomorphic automorphism. This situation has been completely described above (see Proposition 5.5). Thus it remains to consider the case where $G$ is a simple real form of the simple complex group $\widehat{G}$.

\subsubsection{Background}

For the remainder of this paragraph we assume that $G$ is a simple real form of a simple complex group $\widehat{G}$ and consider its action on a $\widehat{G}$-flag manifold $Y=\widehat{G} / \widehat{Q}$. Our particular situation is quite special in that $G$ has a closed orbit $M=G . x_{0}$ of codimension at most two. Before proceeding with this case we summarize here the basic general background which is needed. For details and more information see ([FHW] $)$.

Basic for our applications is the fact that $G$ has exactly one closed orbit in $Y$. In fact $G$ has only finitely many orbits in $Y$ and therefore it has open orbits. Recall that $K$ denotes a maximal compact subgroup of $G$ and that $\widehat{K}$ is its complexification in $\widehat{G}$. There is a important duality between the $G^{-}$ and $\widehat{K}$-orbits in $Y$ (called Matsuki-duality, see [Mat1, Mat2]) which states that to every $G$-orbit there is a unique $\widehat{K}$-orbit which intersects the $G$-orbit in a $K$-orbit and vice versa. In the case of the closed $G$-orbit $M$ this is the simple fact that $K$ acts transitively on $M$ and the $\widehat{K}$-orbit of any point in $M$ is open in $Y$.

There is a very natural $K$-invariant gradient flow on $Y$ which has its critical points at exactly the $K$-orbits which arise in the duality. This flow is tangent to both the $G$ - and the $\widehat{K}$-orbits and, for example, retracts each $\widehat{K}$-orbit onto the $K$-orbit which intersects the $G$-orbit as in the duality theorem. We 
will use this in the case where the flow retracts the open $\widehat{K}$-orbit onto the closed $G$-orbit.

If $D$ is an open $G$-orbit in $Y$, then the above duality states that there is a unique compact (complex) $\widehat{K}$-orbit $C_{0}$ in $D$. It is often of interest to consider it as a point in the cycle space of $D$ or $Y$ and, given a choice of $K$, we therefore refer to it as the base cycle. It can also be characterized as the unique $K$-orbit in $D$ of minimal dimension.

It would be desirable to have $K$-invariant exhaustions of the open orbits which reflect the group theoretic situation. These are known to be available for measurable orbits. If $G$ is of Hermitian type, i.e., if the center of $K$ is positive dimensional (and therefore 1 -dimensional), every open $G$-orbit in every $\widehat{G}$-flag manifold is measurable. Such exhaustions $\rho: D \rightarrow \mathbb{R}^{\geq 0}$ are $q$-convex in the sense that at every point of $D$ the Levi-form $L(\rho)$ has at least $n-q$ positive eigenvalues where $n:=\operatorname{dim} D$. In fact, near the base cycle it is exactly of signature $(n-q, q)$.

In the following two paragraphs we give exact descriptions of the two cases which are relevant for our classification, i.e., where $\operatorname{codim}_{Y} M=1,2$. There are very few possibilities, a fact that does not at all reflect the general situation for actions of real forms on flag manifolds.

\subsubsection{Closed orbits of codimension one}

Here $G$ is a simple real form of $\widehat{G}$ which itself is simple and we assume that the closed $G$-orbit on the flag manifold $Y=\widehat{G} / \widehat{Q}$ is 1 -codimensional. Let us begin with an example which is in fact the only one which arises.

Example. For nonnegative integers $p$ and $q$ with $n+1=p+q$ we consider the action of the real form $G=\mathrm{SU}(p, q)$ of $\widehat{G}=\mathrm{SL}_{n+1}(\mathbb{C})$ on projective space $Y:=\mathbb{P}_{n}(\mathbb{C})$. If we regard a point in $Y$ as a 1 -dimensional subspace $L$ in $\mathbb{C}^{n+1}$, then, restricting the mixed signature Hermitian form $\langle,\rangle_{p, q}$ to $L$, we speak of $L$ as being positive, negative or isotropic, depending on this restriction being positive- or negative-definite or zero. The group $G$ has three orbits in $Y$ : the open sets $D_{+}$and $D_{-}$of positive (resp. negative) lines and the real hypersurface $M$ of isotropic lines. In coordinates, the norm of a 
vector $z=\left(z_{0}, \ldots, z_{n}\right)$ is given by

$$
\|z\|_{p, q}=\sum_{0}^{p-1}\left|z_{i}\right|^{2}-\sum_{p}^{n}\left|z_{i}\right|^{2} .
$$

This corresponds to the splitting $\mathbb{C}^{n+1}=V_{+} \oplus V_{-}$, where $V_{+}:=\left\{z_{p}=\right.$ $\left.\ldots z_{n}=0\right\}$ and $V_{-}=\left\{z_{0}=\ldots=z_{p-1}=0\right\}$ which is invariant by the maximal compact subgroup $K=S\left(U\left(V_{+}\right) \times U\left(V_{-}\right)\right)$of $G$. The base cycles in $D_{+}$and $D_{-}$are $C_{+}:=\mathbb{P}\left(V_{+}\right)$and $C_{-}:=\mathbb{P}\left(V_{-}\right)$, respectively. Since $G$ has three orbits, $\widehat{K}$ does as well, namely the two base cycles and the complement $Y \backslash\left(C_{+} \cup C_{-}\right)$which is the open $\widehat{K}$-orbit containing $M$.

Notice that if $n=2 m-1$ is an odd number, then the symplectic group $\mathrm{Sp}_{2 m}(\mathbb{C})$ acts transitively on $\mathbb{P}_{n}$ and the real form $\operatorname{Sp}(2 p, 2 q)$ which is contained in $\mathrm{SU}(2 p, 2 q)$ is at least a candidate for a smaller real form that acts transitively on $M$. We will show below that this is in fact the case.

Now we prove that these examples are the only ones which arise. First we note that, since the minimal $G$-orbit is a real hypersurface, all other $G^{-}$ orbits are open. Furthermore, the $\widehat{K}$-orbit $\widehat{K} \cdot x_{0}=\widehat{K} / \widehat{H}$ of a point in $M$ is open. Let us consider an affine-rational fibration $\widehat{K} / \widehat{H} \rightarrow \widehat{K} / \widehat{P}$. As usual we $\widehat{K}$-equivariantly compactify this to a $\widehat{K}$-manifold $Y_{1}$ by taking the unique $\widehat{P}$-compactification of the affine fiber and going to the associated bundle over the base. In fact we will see that $\widehat{P} / \widehat{H}$ is just $\mathbb{C}^{*}$, i.e., that $\widehat{K} / \widehat{H}$ has two ends corresponding to two base cycles. If it didn't, then we see that $M$ is the level surface of a $K$-invariant exhaustion and therefore bounds a relatively compact domain in $\widehat{K} / \widehat{H}$ and consequently bounds two domains in $Y$.

Therefore $G$ has exactly two open orbits, $D_{+}$and $D_{-}$, with their boundaries containing $M$. Since $M$ is the unique closed $G$-orbit, these are the only open $G$-orbits in $Y$ and each contains base cycles $C_{+}$and $C_{-}$. Consequently the open $\widehat{K}$-orbit does indeed have two ends and $\widehat{P} / \widehat{H}=\mathbb{C}^{*}$. The compactification $Y_{1}$ therefore has two 1 -codimensional $\widehat{K}$-orbits $E_{+}$and $E_{-}$. The natural identification of the open $\widehat{K}$-orbits in $Y$ and $Y_{1}$ extends to an equivariant birational mapping $\pi: Y_{1} \rightarrow Y$ which, since indeterminacies only exist in codimension two, is in fact regular. As the notation indicates, $E_{+}$is mapped to $C_{+}$and $E_{-}$is mapped to $C_{-}$. Observe that the principal $\mathbb{C}^{*}$-action extends to an action on $Y$ which fixes the cycles pointwise. The $S^{1}$-action stabilizes 
every $K$-orbit and centralizes the $K$-action on $Y$. By our maximality assumption we may assume that $\widehat{G}$ contains this $\mathbb{C}^{*}$ as a subgroup, and one checks that this forces $K$ to contain the $S^{1}$. Therefore $G$ is of Hermitian type!

Now let us define $p-1:=\operatorname{dim} C_{+}$and $q-1:=\operatorname{dim} C_{-}$. Since $G$ is of Hermitian type, we have the $K$-invariant exhaustions $\rho_{+}$and $\rho_{-}$discussed above of the open $G$-orbits $D_{+}$and $D_{-}$. Near $C_{+}$the Levi form $L\left(\rho_{+}\right)$has signature $(n-(p-1), p-1)$ and near $C_{-}$the Levi-form $L\left(\rho_{-}\right)$has signature $(n-(q-1), q-1)$. The essential point now is that the $\mathbb{R}^{>0}$ coming from the central $\mathbb{C}^{*}$-action acts transitively on the $K$-hypersurface orbits. Thus, from the point of view of complex geometry they are all the same. Since the positive eigenvalues of the Levi forms come from the norm in the direction normal to the cycles, we see that the restriction of the Levi forms to the complex tangent spaces of these $K$-orbits has signature $(n-p, p-1)$ and $(n-q, q-1)$ respectively. But when discussing the Levi form near $C_{+}$we are discussing it from the point of view of $C_{+}$being inside a domain defined by $\rho_{+}$. The same is true of $C_{-}$. Since the hypersurfaces are complex analytically the same and the signature of the restricted Levi form is a complex analytic invariant, we have the following fact.

Proposition 5.6. If a $K$-hypersurface orbit in $Y$ is viewed as the boundary of a domain which contains $C_{+}$, then the signature of its Levi form is ( $p-$ $1, q-1)$, where $p-1=\operatorname{dim} C_{+}, q-1=\operatorname{dim} C_{-},(p-1)+(q-1)=n-1$ and $n=\operatorname{dim} Y$.

We wish to show that $G=\mathrm{SU}(p, q)$ and $Y=\mathbb{P}_{n}$ as in the above example. For this we consider the fibrations $E_{+}=\widehat{K} / \widehat{P} \rightarrow \widehat{K} / \widehat{P}_{1}=C_{+}$and $E_{-}=$ $\widehat{K} / \widehat{P} \rightarrow \widehat{K} / \widehat{P}_{1}=C_{-}$. For example, following the fibration $\widehat{K} / \widehat{H} \rightarrow \widehat{K} / \widehat{P}$ of the open $\widehat{K}$-orbit with the first fibration, we have a fibration of the open orbit which extends to a fibration of the disjoint union of the open orbit and $C_{+}$onto $C_{+}$. The fiber is a smooth blow down of zero-section (to a point) of the $\mathbb{C}$-bundle which arises by adding the one end of the $\mathbb{C}^{*}$-bundle over $\widehat{P}_{1} / \widehat{P}$. Thus $\widehat{P}_{1} / \widehat{P}$ is a projective space and a simple dimension count shows that it is $\mathbb{P}_{n-p}$. Analogously we see that $\widehat{P}_{2} / \widehat{P}$ is $\mathbb{P}_{n-q}$. This puts us in a position of being able to prove the main result of this paragraph.

Theorem 5.2. Let $G$ be a simple real form of a simple complex Lie group $\widehat{G}$ whose closed orbit $M$ in the flag manifold $Y=\widehat{G} / \widehat{Q}$ is 1-codimensional. 
Then $Y=\mathbb{P}_{n}$ with $M$ being the manifold of isotropic lines in $Y$. If $\widehat{G}=$ $\mathrm{SL}_{n+1}(\mathbb{C})$, then $G=\mathrm{SU}(p, q)$ where $p+q=n+1$. If $n=2 m-1$ is odd, then the subgroup $\operatorname{Sp}(2 p, 2 q)$, which is a real form of $\operatorname{Sp}_{2 m}(\mathbb{C})$ and is a subgroup of $\mathrm{SU}(2 p, 2 q)$, also acts transitvely on $M$.

Proof. We will show that the diagonal $\widehat{K}$-action on $\widehat{K} / \widehat{P}_{1} \times \widehat{K} / \widehat{P}_{2}$ coming from the two fibrations of $\widehat{K} / \widehat{P}$ defines an isomorphism

$$
\widehat{K} / \widehat{P} \rightarrow \widehat{K} / \widehat{P}_{1} \times \widehat{K} / \widehat{P}_{2} \cong \mathbb{P}_{p-1} \times \mathbb{P}_{q-1}
$$

For this, observe that the fibers $\widehat{P}_{1} / \widehat{P}=\mathbb{P}_{q-1}$ and $\widehat{P}_{2} / \widehat{P}=\mathbb{P}_{p_{1}}$ are transversal to one another, because they correspond to the negative and positive eigenspaces of the Levi form. So, for example, the closed $\widehat{P}_{2}-$ orbit $\widehat{P}_{2} / \widehat{P}$ in $\widehat{K} / \widehat{P}$ is mapped biholomorphically onto the base of $\widehat{K} / \widehat{P} \rightarrow \widehat{K} / \widehat{P}_{1}$. Thus, as desired, the bases of the the fibrations are $\mathbb{P}_{p-1}$ and $\mathbb{P}_{q-1}$ respectively.

Now, modulo the ineffectivity of the $\widehat{K}$-action on the base $\widehat{K} / \widehat{P}_{1}$, a semisimple part of $\widehat{P}_{1}$ is at most $\mathrm{SL}_{p-2}(\mathbb{C})$. Since we may assume without loss of generality that $q \geq p$, this is not enough to be the semisimple part of a group which acts transtively on the fiber $\widehat{P}_{1} / \widehat{P}$. Thus a semisimple part of the ineffectivity of the $\widehat{K}$-action on $\widehat{K} / \widehat{P}_{1}$ must bring up the semisimple part of $P_{1}$ to a group which can act transitively on $\mathbb{P}_{q_{1}}$. The only possibility for this is that this ineffectivity itself acts transitively on the fiber $\mathbb{P}_{q-1}$. This proves (5.4.2).

To prove the final statement we must only note that the line bundles on $\mathbb{P}_{p-1} \times \mathbb{P}_{q-1}$ are just the tensor products of pullbacks of powers of hyperplane section bundles from the factors. The only possibility to blow down the 0and $\infty$-section as we do here (obtaining a smooth manifold) is the bundle with Chern number $(1,1)$. Blowing this down we obtain the projective space $\mathbb{P}_{n}$.

Finally, we must show that the only groups which do the job are $\mathrm{SU}(p, q)$, as a real from of $\mathrm{SL}_{n+1}(\mathbb{C})$ or, in the case where $n=2 m-1$ is odd, $\operatorname{Sp}(2 p, 2 q)$ as a real form of $\operatorname{Sp}_{2 m}(\mathbb{C})$. Since the manifold $M$ is known to be the bundle space of the $S^{1}$-principal bundle of the $(1,1)$-bundle over $\mathbb{P}_{p-1} \times \mathbb{P}_{q-1}$, if there is to be a subgroup of $\mathrm{SU}(p, q)$ which acts transtively on $M$, its maximal compact subgroup must act transitively on this product. Changing the notation, the maximal compact subgroup $U S p_{2 p} \times U S p_{2 q}$ of the real form $\operatorname{Sp}(2 p, 2 q)$ of 
$\mathrm{Sp}_{2 m}$ does indeed act transitively on this product. Further, the $S^{1}$-bundle in question is topologically nontrivial; so this maximal compact subgroup must act transitively there as well. Thus, in this case the subgroup $\operatorname{Sp}(2 p, 2 q)$ of $\mathrm{SU}(2 p, 2 q)$ also acts transitively on $M$.

\subsubsection{Closed orbits of codimension two}

Again we remind the reader of the situation. Here $G$ is a semisimple real form of the semisimple complex group $\widehat{G}$. We assume that $G$ has a closed orbit $M$ of codimension two in the flag manifold $Y=\widehat{G} / \widehat{Q}$ and split the manifold $Y=Y_{1} \times \cdots \times Y_{m}$ according to the factors $\widehat{G}_{i}$ which are complexifications of the simple factors $G_{i}$ of $G$. If we have a situation where the simple factor $G_{i}$ is a complex group and its complexification is $G_{i} \times G_{i}$ and $G_{i}$ does not act transitively on the flag manifold $Y_{i}$, then $M$ splits as a product of the other $Y_{j}$ with $M_{i} \hookrightarrow Y_{i}=\mathbb{P}_{n} \times \mathbb{P}_{n}$ as in Proposition 5.5. This case being handled, we may assume that all of the $\widehat{G}_{i}$ are simple.

If we split off all factors $Y_{i}$ where $G_{i}$ acts transitively, then we are left with two possibilities: $Y=Y_{1} \times Y_{2}$, where the closed $G_{i}$-orbit is a real hypersurface as described in the previous paragraph, and the case which is to be handled in this paragraph, where both $G$ and $\widehat{G}$ are simple. Thus we assume that we have this latter situation. The following is then the main result of this paragraph.

Theorem 5.3. If $G$ and $\widehat{G}$ are simple and the closed $G$-orbit $M$ in the flag manifold $\widehat{G} / \widehat{Q}$ is 2-codimensional, then $G=\mathrm{SL}_{3}(\mathbb{R}), \widehat{G}=\mathrm{SL}_{3}(\mathbb{C})$, the flag manifold $\widehat{G} / \widehat{Q}$ is projective space $\mathbb{P}_{2}(\mathbb{C})$ and the closed $G$-orbit is the set of real points $M=\mathbb{P}_{2}(\mathbb{R})$.

We will prove this by induction on the dimension $n=\operatorname{dim} Y$. This begins with $n=2$ where the only possibility for $Y$ is $\mathbb{P}_{2}(\mathbb{C})$. The only complex group $\widehat{G}$ which acts transitively on $\mathbb{P}_{2}(\mathbb{C})$ is (up to coverings) $\mathrm{SL}_{3}(\mathbb{C})$ which only has $\mathrm{SL}_{3}(\mathbb{R})$ and $\mathrm{SU}(2,1)$ as its real forms. The latter case has been discussed above and the former is exactly the case which occurs in the theorem. For the remainder of this paragraph we will operate under the induction assumption. In other words, we will be dealing with a flag manifold of dimension $n$ where the assumption of the theorem holds and will assume that the theorem holds for flag manifolds of every lower dimension. 
Proposition 5.7. The group $\widehat{Q}$ is a maximal parabolic subgroup of $\widehat{G}$.

Proof. If $\widehat{Q}$ is not a maximal parabolic, then we have a nontrivial fibration $\widehat{G} / \widehat{Q} \rightarrow \widehat{G} / \widehat{Q}_{1}$ and we study the various possible situations for the $G$-action on the base. If this action is transitive, our situation reproduces itself in the fiber. Here, however, we must be careful with the induction assumption. If the fiber group $G_{1}=G \cap \widehat{Q}_{1}$ is acting as in the theorem, then, modulo ineffectivity this is $\mathrm{SL}_{3}(\mathbb{R})$ which has a maximal compact subgroup $\mathrm{SO}_{3}(\mathbb{R})$. In this case we check the possibilities given to us by Onishchik ([01, O2]) and see that a maximal compact subgroup in the $G_{1}$-isotropy of the base never has an $\mathrm{SO}_{3}(\mathbb{R})$-quotient. The other two possibilities, where we in fact can not apply the induction assumption, are handled analogously. These are where the fiber is a product of hypersurface orbits or where it is an orbit of a complex simple group which is acting as a real form. The groups which are needed for this are simply not to be found as quotients of the $G_{1}$-isotropy given to us by Onishchik ([01, O2]).

If $G$ does not act transitively on the base and the situation of the theorem is reproduced, then $G=\mathrm{SL}_{3}(\mathbb{R})$, the base is $\mathbb{P}_{2}(\mathbb{C})$ and the only possibility for $Y$ is the manifold of full flags where the closed $G$-orbit is 3-codimensional. Thus this case also doesn't occur.

Finally, if the closed $G$-orbit in $\widehat{G} / \widehat{Q}_{1}=\mathbb{P}_{n}(\mathbb{C})$ is a real hypersurface, then we directly check that neither of the possibilities for a real form $G$ acts with 2 -codimensional closed orbit on the bigger flag manifold $\widehat{G} / \widehat{Q}$.

We now turn to an analysis of the open $\widehat{K}$-orbit $\Omega$ in $Y$. For this we let $E:=\operatorname{bd}(\Omega)$ be its boundary. For example, we wish to show that the generic $K$-orbit in $\Omega$ is a real hypersurface. To see this, observe that since the gradient flow which realizes duality and retracts $\Omega$ to $M$ is $K$-invariant, every $K$-orbit in $\Omega$ fibers over $M$. In particular, if the generic $K$-orbit in $\Omega$ is not a hypersurface, then every $K$-orbit in $\Omega$ is 2 -codimensional. Let us show that this is not the case.

In the following we say that points in a $\widehat{K}$-orbit $\mathcal{O}_{1}$ are only accessible from an orbit $\mathcal{O}_{2}$ if the only orbit which has $\mathcal{O}_{1}$ in its closure is $\mathcal{O}_{2}$.

Lemma 5.1. Let $\mathcal{O}$ be a $\widehat{K}$-orbit in $E$ and let $y_{0} \in \mathcal{O}$ only be accessible by the open orbit $\Omega$. Assume that all $K$-orbits in $\Omega$ are 2-codimensional over $\mathbb{R}$. Then the codimension of K.y in $\mathcal{O}$ is at most one. 
Proof. This follows from the fact that the isotropy group $K_{y_{0}}$ stabilizes a polydisk transversal to $\mathcal{O}$ where its orbits are at most 1-codimensional.

Now if $y_{0} \in \mathcal{O}$ is only accessible from the open orbit, then every point $y \in \mathcal{O}$ has this property. Thus we have the following remark.

Corollary 5.1. If $\mathcal{O}$ contains a point which is only accessible from $\Omega$, then $\mathcal{O}$ is not affine.

Proof. If $\mathcal{O}$ is affine and the semisimple part of $\widehat{K}$ acts nontrivially on $\mathcal{O}$, then the minimal $K$-orbit in $\mathcal{O}$ is not 1 -codimensional. Recall that unless $G$ is of Hermitian type, then $K$ is semisimple and even in the Hermitian case it has only a 1 -dimensional center. So if the semisimple part acted trivially on $\mathcal{O}$, then $\mathcal{O} \cong \mathbb{C}^{*}$ and $\widehat{K}$ would have a fixed point on the boundary. The $G$-orbit of this cycle would be the bounded symmetric domain in $Y$. But since $Y \neq \mathbb{P}_{1}$, we know that the minimal $K$-orbit in the bounded symmetric domain is larger than 1-dimensional.

Now suppose $E$ contains irreducible components which are 1-codimensional and let $E_{0}$ be the union of these components. Since $\widehat{Q}$ is maximal, this divisor is ample and the complement is Stein. Let $\mathcal{O}$ be a maximal dimensional orbit in $E$ in the complement of $E_{0}$. It is clearly only accessible in the above sense and therefore the generic $K$-orbits in $\mathcal{O}$ are real hypersurfaces in $\mathcal{O}$. Since the maximal dimensional orbit on the boundary of $\mathcal{O}$ is only accessible from $\mathcal{O}$, it follows by the same argument that the $K$-orbits there are 0 -codimensional, i.e., the $\widehat{K}$-orbits on the boundary of $\mathcal{O}$ are even closed in $Y$. Since $E_{0}$ is an ample divisor, such orbits must have nonempty intersection with $E_{0}$ and consequently they are contained in $Y$. Thus we have the following situation.

Proposition 5.8. Either $E_{0}=\emptyset$, i.e., the boundary $E$ of the open $\widehat{K}$-orbit in $Y$ is everywhere at least ${ }^{2}$-codimensional, or $E_{0}=E$ is an ample divisor.

Proof. We have just seen that if $E_{0}$ is nonempty, but $E_{0} \neq E$, then every orbit $\mathcal{O}$ in the complement of $E_{0}$ in $E$ is closed in the complement of $E_{0}$ in $Y$. Since $E_{0}$ is ample, this means that every such $\mathcal{O}$ is affine. But this was ruled out above (recall we are still operating under the assumption that every $K$-orbit in $\Omega$ is 2 -codimensional). 
Finally, we come to the key point! If $E_{0}=\emptyset$, then the real codimension of $E$ in $Y$ is at least four. Hence the homology groups $H_{2}(\Omega)$ and $H_{2}(Y)$ agree. Since $\widehat{Q}$ is maximal, $b_{2}(Y)=1$. This information allows us to prove the following result.

Theorem 5.4. The boundary $E$ is an ample divisor in $Y$ and the generic $K$-orbit in $\Omega$ is a real hypersurface.

Proof. We have seen above that if $E$ is not an ample divisor, then $H_{2}(\Omega)=$ $H_{2}(Y)=\mathbb{Z}$. Now the fiber $\widehat{P} / \widehat{H}$ is an affine homogeneous space which is either a $\mathbb{C}^{*}$-bundle over an affine symmetric space or is itself $\left(\mathbb{C}^{*}\right)^{2}$. In the former cases there are always $K$-orbits which are of codimension more than two. So if every $K$-orbit is 2 -codimensional, then this fiber is $\left(\mathbb{C}^{*}\right)^{2}$. In this case $\widehat{P}$ is represented on the fiber as $\left(\mathbb{C}^{*}\right)^{2}$ and is therefore not a maximal parabolic. Thus $b_{2}\left(Y_{1}\right) \geq 2$.

Finally, $M$ is a strong deformation retract of $\Omega$ and, even in the Hermitian case where there can be a contribution from the center of $K, \pi_{1}(M)$ is either $\mathbb{Z}$ or finite. Thus, if all $K$-orbits are 2 -codimensional and $E_{0} \neq E$, then an application of the homotopy sequence to the fibration $Y \rightarrow Y_{1}$ shows that $b_{2}(Y) \geq 2$ ! This contradiction means that either $E$ is an ample divisor or the generic $K$-orbits in $\Omega$ are hypersurfaces or both. We claim both.

To see this, observe that if the generic $K$-orbits are hypersurfaces, then the complement of $\Omega$ consists of the union of the closed $\widehat{K}$-orbits, i.e., the cycles. Nagano's theorem states that in this situation there are at most two $K$-orbits in $Y$ which are lower-dimensional than hypersurfaces. Since $M$ is already one of them, it follows that there is exactly one cycle in this situation. Furthermore, $M$ is the minimal $K$-orbit in $\Omega$. Since $M$ is $2-$ codimensional, the fiber of the affine-rational fibration is therefore also 2 dimensional, i.e., it is either the affine 2-dimensional quadric or its 2:1quotient which is $\mathbb{P}_{2}$ with a quadric curve removed. In any case this fiber is uniquely equivariantly compactified by adding a copy of $\mathbb{P}_{1}$ to obtain a two orbit manifold which is mapped regularly to $Y$ with the lower-dimensional orbit which was constructed by compactifying the fiber being mapped to $E$. But this map blows down the curve that was added to the affine fiber unless the cycle itself was already a divisor! Thus, if the generic $K$-orbit in $\Omega$ is a hypersurface, then $E$ is indeed an ample divisor. 
Conversely, if $E$ is an ample divisor, then $\Omega$ is already affine and consequently $M$ is totally real and therefore is 2-dimensional. Since this situation is completely understood, i.e., we are at the beginning step of the induction, we actually prove the complete classification result in this case!

As the reader has undoubtedly noticed, the proof of Theorem 5.3 is now complete. Since $E$ is now known to be an ample divisor, just as in the last step above, we see that $Y=\mathbb{P}_{2}(\mathbb{C})$ and $G=\mathrm{SL}_{3}(\mathbb{R})$ is acting on $Y$ as usual.

\subsection{Classification theorem}

Above we have proved classification results in all situations which can arise where $M=G / H=G . x_{0}$ is a compact Cauchy-Riemann homogeneous space which is realized as a $G$-orbit in some projective space. Here we put this classification together in a systematic way.

In our particular situation we automatically have the globalization $X=$ $\widehat{G} / \widehat{H}=\widehat{G} \cdot x_{0}$ on the ambient projective space. Recall that the radical $T$ of $G$ is a compact torus and that $G=T \cdot S$ is a product (with possible finite intersection) of $T$ with its semisimple part $S$. The complex group $\widehat{G}=\widehat{T} \cdot \widehat{S}$ splits accordingly and $T$ is a real form of its radical $\widehat{T}=\left(\mathbb{C}^{*}\right)^{n}$. However, it is quite possible that one or more of the simple factors of $S$ is a complex simple factor of $\widehat{S}$. On the other hand, we have shown that the orbits of such a factor are compact and that $M$ is a $G$-equivariant $\mathrm{CR}$-product $M=M^{\prime} \times Z$, where $Z=\widehat{G}_{1} / \widehat{Q}_{1}$ is the compact complex homogeneous rational manifold which is the orbit of the product of such factors. Since we are dealing with algebraic groups, the situation reproduces itself in the sense that $M^{\prime}$ is such an orbit,

but with the advantage that now $G$ is a real form of $\widehat{G}$. At various stages of the classification we will see that there is such a compact complex factor $Z$ and therefore it is convenient to refer to $M$ as having a certain form up to compact complex factors. This means that we are referring to the manifold $M^{\prime}$.

Thus, after eliminating such compact factors we begin with an affine-rational fibration

$$
X=\widehat{G} / \widehat{H} \rightarrow \widehat{G} / \widehat{Q}=Y
$$


which is assumed to be minimal in the sense that there is no intermediate fibration where the base is compact. This restricts to a $\mathrm{CR}$-fibration of $M=G / H \rightarrow G / Q=N$. At the level of the complex groups the base $\widehat{G} / \widehat{Q}$ is a classical object where for example the structure of $\widehat{Q}$ is well-understood. Above we have explained all possibilities for the fiber $\widehat{Q} / \widehat{H}$ and given $\widehat{Q}$ in root-theoretic terms it is not difficult to determine the possibilities for $\widehat{H}$. Let us now consider the possibilities for how $M$ is embedded in $X$.

\section{Compact spherical type}

Above it was shown that if $G$ acts transitively on the base $\widehat{G} / \widehat{Q}$, i.e.,

$$
Y=\widehat{G} / \widehat{Q}=G / Q=N,
$$

then $G$ is compact. We refer to the $\mathrm{CR}$-homogeneous manifolds $M=$ $G / H \hookrightarrow \widehat{G} / \widehat{H}=X$ which arise in this way as being of compact spherical type. They are constructed as follows.

Start with a compact group $G$ with complexification $\widehat{G}$ and consider a parabolic subgroup $\widehat{Q}$ with $Y:=\widehat{G} / \widehat{Q}$. Without loss of generality it may be assumed that the root description of $\widehat{Q}$ is based around a maximal complex torus $\widehat{T}$ in $\widehat{Q}$ which is the complexification of a maximal compact torus in $G$. This root description defines a maximal reductive subgroup $\widehat{L}$ of $\widehat{Q}$ (a Levi-factor $\widehat{Q}_{r}$ ) which is the complexification of a compact subgroup $L$ of the compact group $K$. Now one checks the list of possible affine-spherical fibers $\widehat{F}$ (see the above table) and determines whether or not there is one where $\widehat{L}$ can be represented (with possible ineffectivity) as the reductive group acting on $\widehat{F}$.

Recalling that $\widehat{Q}=\widehat{L} \ltimes \widehat{Q}_{u}$, one then has an action of $\widehat{Q}$ on $\widehat{F}$ and the $\widehat{G}$-algebraic homogeneous space

$$
X=\widehat{G} / \widehat{H}=\widehat{G} \times \widehat{Q} \widehat{F} .
$$

Here we choose $x_{0} \in X$ to be a point in the standard embedding of the fiber $\widehat{F}$ in $\widehat{G} \times{ }_{\widehat{Q}} \widehat{F}$ where the orbit L. $x_{0}$ is of codimension one or two, depending on the rank of $\widehat{F}$. The CR-homogeneous space of interest is then $M:=G . x_{0}$. It should be emphasized that there is an open dense set $X_{\text {gen }}$ in $X$ where any two $G$-orbits are equivariantly, real-analytically diffeomorphic. We refer to such orbits as being generic. Each of these will inherit invariant CR-structure 
from the ambient manifold. One should expect, however, any two of these orbits are not $\mathrm{CR}$-equivalent.

\section{Basic models with noncompact symmetry groups}

Now we turn to the basic building blocks of projective Cauchy-Riemann manifolds $M:=G / H$ of codimension one or two which are homogeneous under a noncompact symmetry group. These are orbits of simple real forms $G$ of complex semisimple groups in $\widehat{G}$-flag manifolds $X=\widehat{G} / \widehat{H}$. As was shown above, in this case there are two infinite series of examples and one exceptional case. The following is a quick summary of these basic models.

\section{Mixed signature quadrics}

Here $X=Y=\mathbb{P}_{n}(\mathbb{C})$ and $M=M_{p, q}$ is the manifold of isotropic lines of the standard Hermitian norm of signature $(p, q)$. We refer to the real hypersurface $M$ as a mixed signature quadric. Its stabilizer in the automorphism group of $\mathbb{P}_{n}$ is the group $\mathrm{SU}(p, q)$ which is a real form of $\mathrm{SL}_{n+1}(\mathbb{C})$. In the case where $n+1$ is even where we are considering the form of signature $(2 p, 2 q)$ the real form $\operatorname{Sp}(2 p, 2 q)$ of $\operatorname{Sp}_{n+1}(\mathbb{C})$ also acts transitively on $M$. As is proven above, if $G$ is a noncompact Lie group acting on $M$ under the standard assumptions of this section and $M$ is 1-codimensional, then, up to compact factors, $M$ is a mixed signature quadric $M_{p, q}$ and $G$ one of the groups just mentioned.

\section{Twisted diagonal actions on products of projective space}

If $G$ itself is a simple complex Lie group and $\varphi: G \rightarrow G$ is an antiholomorphic automorphism, then we embed it in its complexification $\widehat{G}=G \times G=G_{1} \times G_{2}$ by the twisted diagonal map $(g, \varphi(g))$. If $Q_{1}$ is a parabolic subgroup of $G_{1}$, then $Q_{2}=\varphi\left(Q_{1}\right)$ is parabolic in $G_{2}$ and $G$ acts by this embedding on the homogeneous rational manifold $X=Y=\widehat{G} / \widehat{Q}=G_{1} / Q_{1} \times G_{2} / Q_{2}$. This is a context which should be studied further. However, in our case there is only one situation of interest which arises when $G_{1}=G_{2}=\mathrm{SL}_{n+1}(\mathbb{C})$ and $Q_{1}$ is the standard isotropy group for the $G_{1}$-action on $\mathbb{P}_{n}(\mathbb{C})$. In the case of the antiholomorphic automorphism which is defined by complex conjugation, i.e., $\varphi(A)=\bar{A}, Q_{2}=Q_{1}$ and both factors of $X$ are the same. The closed $G$-orbit in question is just the antiholomorphic diagonal $M=\{([z],[w]) \in$ 
$\left.\mathbb{P}_{n} \times \mathbb{P}_{n} ; z=\bar{w}\right\}$. Thus in our case where $M$ has been assumed to be at most 2 -codimensional there is only one examples of interest, i.e., for $n=1$.

Up to conjugation, the only other antiholomorphic automorphism of $\mathrm{SL}_{n+1}(\mathbb{C})$ is given by composing the map $\varphi$ above with the holomorphic outer automorphism $A \rightarrow\left(A^{-1}\right)^{t}$. Thus $X=\mathbb{P}_{n} \times \mathbb{P}_{n}^{*}$, where by abuse of notation $\mathbb{P}_{n}^{*}$ denotes $\mathbb{P}\left(\left(\mathbb{C}^{n}\right)^{*}\right)$. The closed orbit in this case can be described in standard coordinates as

$$
M=\left\{([z],[w]) \in \mathbb{P}_{n} \times \mathbb{P}_{n} ; z^{t} \bar{w}=0\right\} .
$$

It is the image by complex conjugation in the second factor of the complex hypersurface $\left\{([z],[w]) ; z^{t} w=0\right\}$ and therefore, independent of $n$ is 2-codimensional. We refer to these $\mathrm{CR}$-homogeneous spaces as those which arise by twisted diagonal actions on products of projective spaces. In the case $n=1$ the projective space $\mathbb{P}_{1}$ is holomorphically equivariantly isomorphic to its dual. Thus the above case of the antiholomorphic diagonal in $\mathbb{P}_{1} \times \mathbb{P}_{1}$ is the same as the case which was just discussed in the special situation where $n=1$. Thus there is only one series of manifolds $M_{n}$ which arise by twisted diagonal actions.

\section{The real points in $\mathbb{P}_{2}(\mathbb{C})$}

If we equip $\mathbb{C}^{n}$ with its standard real structure, then the associated projective space $X=\mathbb{P}_{n}(\mathbb{C})=\mathbb{P}\left(\mathbb{C}^{n}\right)$ is defined over the reals and its set of real points is just the projectivization of $\mathbb{P}\left(\mathbb{R}^{n}\right)=\mathbb{P}_{n}(\mathbb{R})$. The real form $G=\mathrm{SL}_{n+1}(\mathbb{R})$ of $\widehat{G}=\mathrm{SL}_{n+1}(\mathbb{C})$ has exactly two orbits on $\mathbb{P}_{n}(\mathbb{C})$, namely $\mathbb{P}_{n}(\mathbb{R})$ and its complement. Since the case of $n=1$ is a mixed signature quadric for the group $\mathrm{SU}(1,1)$, we don't consider it here. So the only case which occurs in our context of interest is the closed orbit $\mathbb{P}_{2}(\mathbb{R})$ of $G=\mathrm{SL}_{3}(\mathbb{R})$ in $\mathbb{P}_{2}(\mathbb{C})$.

The following summarizes the classification results on basic models with noncompact symmetry groups in the standard situation of this section.

Theorem 5.5. If $G$ is simple and noncompact, then $M$ is one of the following:

1. A mixed signature quadric $M_{p, q}$ of codimension one.

2. The 2-codimensional closed orbit $M_{n}$ in $\mathbb{P}_{n} \times \mathbb{P}_{n}^{*}$ of $G=\mathrm{SL}_{n+1}(\mathbb{C})$ acting by an the antiholomorphically twisted diagonal embedding $A \rightarrow$ $\left(A,\left(\bar{A}^{-1}\right)^{t}\right)$ in $\mathrm{SL}_{n+1}(\mathbb{C}) \times \mathrm{SL}_{n+1}(\mathbb{C})$. 
3. The exceptional example of the real projective plane $\mathbb{P}_{2}(\mathbb{R})$ embedded as usual as the closed orbit of $G=\mathrm{SL}_{3}(\mathbb{R})$ on $\mathbb{P}_{2}(\mathbb{C})$.

\section{Splitting Theorem}

The following result allows us to put together a clean statement of our classification result. In order to put it in perspective one should recall that if the real form $G$ acts transitively on be base $\widehat{G} / \widehat{Q}$, then it is compact.

Proposition 5.9. Let $G_{1}$ be a noncompact simple factor of $G=G_{1} \cdot G_{2}$ with complexification $\widehat{G}_{1}$. Denote the corresponding splittings of the complexified group and base by $\widehat{G}=\widehat{G}_{1} \cdot \widehat{G}_{2}$ and $X=\widehat{G} / \widehat{Q}=\widehat{G}_{1} / \widehat{Q}_{1} \times \widehat{G}_{2} / \widehat{Q}_{2}$. Assume that $G_{1}$ does not act transitively on $\widehat{G}_{1} / \widehat{Q}_{1}$. Then $X$ splits $\widehat{G}$-equivariantly as a product $X=\widehat{G}_{1} / \widehat{Q}_{1} \times \widehat{G}_{2} / \widehat{H}_{2}$, where $\widehat{Q}_{1}=\widehat{H} \cap \widehat{G}_{1}$ and $\widehat{H}_{2}=\widehat{H} \cap \widehat{G}_{2}$. The CR-homogeneous manifold splits $G$-equivariantly $M=M_{1} \times M_{2}$ along the same lines.

Before turning to the proof, let us formulate our main classification theorem which follows directly from the splitting theorem and the classification results proved above. In it we assume that we have the standard situation of this section where in particular $M=G / H$ is at most 2-codimensional in $X=$ $\widehat{G} / \widehat{H}$ and the latter has an affine-rational fibration $X=\widehat{G} / \widehat{H} \rightarrow \widehat{G} / \widehat{Q}=Y$. The induced fibration of $M$ is denoted by $M:=G / H \rightarrow G / Q=N$

Theorem 5.6. If $N$ is 2-codimensional in the base, then $M=N, X=Y$ and $M$ one of the following:

1. A product $M=M_{p, q} \times M_{p^{\prime}, q^{\prime}}$ of mixed signature quadrics with the groups $G=G_{1} \times G_{2}$ and $\widehat{G}=\widehat{G}_{1} \times \widehat{G}_{2}$ and complex model $X=\widehat{G}_{1} / \widehat{Q}_{1} \times \widehat{G}_{2} / \widehat{Q}_{2}$ splitting accordingly.

2. One of the series $M_{n}$ which is the closed $\mathrm{SL}_{n+1}(\mathbb{C})$-orbit in $\mathbb{P}_{n}(\mathbb{C}) \times$ $\mathbb{P}_{n}(\mathbb{C})^{*}$, where $G=\mathrm{SL}_{n+1}(\mathbb{C})$ is acting by the antiholomorphically twisted diagonal representation.

3. The closed orbit $M=\mathbb{P}_{2}(\mathbb{R})$ of $G=\mathrm{SL}_{3}(\mathbb{R})$ in $\mathbb{P}_{2}(\mathbb{C})$.

If $N$ is 1-codimensional in the base and $M$ is 1-codimensional in $X$, then $X=Y=\mathbb{P}_{n}(\mathbb{C})$ and $M$ is a mixed signature quadric with $G$ either $\operatorname{SU}(p, q)$ or $\operatorname{Sp}(2 p, 2 q)$. 
If $N$ is 1-codimensional in the base, and $M$ is 2-codimensional in $X$, then $M=M_{1} \times M_{2}$ splits as a product of a mixed signature quadric $M_{1}$ and a manifold $M_{2}$ of compact spherical type. This splitting is $G$-equivariant and is defined by a splitting $X=\widehat{G} / \widehat{H}=\widehat{G}_{1} / \widehat{Q}_{1} \times \widehat{G}_{2} / \widehat{H}_{2}=X_{1} \times X_{2}$, where $M_{1}$ is the mixed signature quadric orbit of the noncompact real form $G_{1}(\mathrm{SU}(p, q)$ or $\operatorname{Sp}(2 p, 2 q)$ ) in $Y_{1}=\mathbb{P}_{n}$. The group $G_{2}$ is compact with $M_{2}$ being a 1codimensional $G_{2}$-orbit in $X_{2}=\widehat{G}_{2} / \widehat{H}_{2}$

If $G$ acts transitively on the base, i.e., if $N=Y$, then $M$ is of compact spherical type.

We now turn to the

Proof of Proposition 5.9. It is only necessary to handle the case where the image $N=G / Q$ in the base is the product of the closed orbit $N_{1}=G_{1} / Q_{1}$ of the simple factor $G_{1}$ in $Y_{1}=\widehat{G}_{1} / \widehat{Q}_{1}$ with the other factor $Y_{2}=\widehat{G}_{2} / \widehat{Q}_{2}=$ $G_{2} / Q_{2}=N_{2}$ of the rational homogeneous base. For this we consider the fiber $\widehat{F}=\widehat{G}_{1} \times \widehat{Q}_{2} / \widehat{H}$ of the fibration $\widehat{G}_{1} \times \widehat{G}_{2} / \widehat{H} \rightarrow \widehat{G}_{2} / \widehat{Q}_{2}$ over this factor. We have the intermediate fibration

$$
\widehat{G}_{1} \times \widehat{Q}_{2} / \widehat{H} \rightarrow \widehat{G}_{1} \times \widehat{Q}_{2} / \widehat{H} \widehat{Q}_{2}=\widehat{G}_{1} / \widehat{H}_{1} \rightarrow \widehat{G}_{1} / \widehat{Q}_{1} .
$$

From the minimality assumption on the original affine-rational fibration it follows that $\widehat{H}_{1}=\widehat{Q}_{1}$.

The only case of interest is where the fiber of the induced fibration of the CR-manifold $F$ is a real hypersurface in its complexification $\widehat{H} \widehat{Q}_{2}$. If the real group acting on this fiber were noncompact, then our classification would imply that $\widehat{H} \widehat{Q}_{2} / \widehat{H}$ would be compact. This would mean that $X=Y$ is compact and $M=M_{1} \times M_{2}$ would be a product of mixed signature quadrics. This is of course possible, but, as we remarked at the outset of this proof, it is only necessary to handle the case where only one noncompact factor of $G$ has a noncompact orbit in the base $\widehat{G} / \widehat{Q}$.

In summary, the above shows that we have a fibration

$$
\widehat{G}_{1} \times \widehat{Q}_{2} / \widehat{H} \rightarrow \widehat{G}_{1} / \widehat{Q}_{1}=\mathbb{P}_{n}(\mathbb{C}),
$$

where the real group acting on the base is either $\mathrm{SU}(p, q)$ or $\mathrm{SU}(2 p, 2 q)$. If the mixed signature quadric in the base is the orbit $N_{1}=G_{1} \cdot y_{0}=G_{1} / Q_{1}$, then we 
know that $Q_{1}$ acts as a compact group on the fiber. Since connected solvable groups of compact groups are Abelian, this implies that the commutator group of every noncompact solvable subgroup of $Q_{1}$ acts trivially on the fiber. In particular $\widehat{Q}_{1}$ contains a normal subgroup $\widehat{I}$ which contains every such group. An explicit check of the form of $Q_{1}$ shows that the only possibility for $\widehat{I}$ is $\widehat{Q}_{1}$ itself. For example, if one considers the stabilizer in $G_{1}$ of a $2^{-}$ dimesional complex subspace of $\mathbb{C}^{n}$ of signature $(1,1)$, then the isotropy of this copy of $\mathrm{SU}(1,1)$ at the base point of $N_{1}$ is a real Borel group whose commutator is contained in no proper normal subgroup of $\widehat{Q}_{1}$.

The proof is now complete, because the above shows that $\widehat{Q}_{1}$ acts trivially on the fiber and therefore is contained in $\widehat{H}$. This implies that $X=X_{1} \times X_{2}=$ $\widehat{G}_{1} / \widehat{Q}_{1} \times \widehat{G}_{2} / \widehat{H}_{2}$ and $M$ splits accordingly.

\subsection{Application to globalization}

Although our goal here is to show that under certain assumptions globalization is possible, we begin by observing that there can be substantial problems caused by the presence of certain 3-dimensional CR-hypersurfaces.

\subsubsection{On the role of the affine quadric}

Here we give several examples to indicate how the 2-dimensional affine quadric $\mathrm{SL}_{2}(\mathbb{C}) / T$, where $T$ is the subgroup of diagonal matrices, can play a role in hindering globalization. This is just the tangent bundle of $S^{2}$ where, except for the 0 -section, every $\mathrm{SO}_{3}(\mathbb{R})$ orbit is 1-codimensional and just a copy of $\mathrm{SO}_{3}(\mathbb{R})$ itself. Thus the CR-structures on these orbits are left-invariant hypersurface structures on the group itself. The manifolds which cause the difficulty in globalization are the universal covers of these manifolds, i.e., left-invariant CR-hypersurface structures on the group $\mathrm{SU}_{2}$. These nonglobalizable structures can be built into other CR-homogeneous spaces in various ways in order to construct other nonglobalizatable examples. Let us discuss several such examples.

We begin by considering $\mathrm{SL}_{2}(\mathbb{C})$ represented on $\mathbb{C}^{N}$ as a direct sum of two irreducible representations, one where - Id acts as the identity, i.e., a representation of even highest weight, and one where - Id acts as - Id. Consider the affine symmetric space $X:=\mathrm{SL}_{2}(\mathbb{C}) / D$, where $D \cong \mathbb{C}^{*}$ is the space 
of diagonal matrices in $\mathrm{SL}_{2}(\mathbb{C})$, and equip $\mathrm{SU}_{2}$ with the left-invariant $\mathrm{CR}$ structure which comes from one of its real hypersurface orbits in $X$.

As was noted in the example at the beginning of section three, with this structure $M=\mathrm{SU}_{2}$ can not be globalized. On the other hand, the 2:1 quotient $M / \pm \mathrm{Id}$ is beautifully globalized in $X$. Here we modify this example slightly to obtain a 1-codimensional $\mathrm{CR}$-homogeneous space which is much more difficult to handle.

For this let $\mathrm{SL}_{2}(\mathbb{C})$ be represented on $\mathbb{C}^{N}$ as a direct sum $\rho$ of two irreducible representations, one where $-\mathrm{Id}$ acts as the identity, i.e., a representation of even highest weight, and one where - Id acts as - Id, and let $\widehat{G}:=\mathbb{C}^{N} \rtimes_{\rho}$ $\mathrm{SL}_{2}(\mathbb{C})$ be the resulting complex Lie group. We define $G=\mathbb{C}^{N} \rtimes \mathrm{SU}_{2}$, where $\mathrm{SU}_{2}$ is equipped with the $\mathrm{CR}$-structure defined above. Finally, let $\Gamma$ be a full lattice in $\mathbb{C}^{N}$ so that $\mathbb{C}^{N} / \Gamma=T$ is a complex torus. Due to the choice of the mixed representation, $-\mathrm{Id} \in \mathrm{SU}_{2}$ does not normalize most $\Gamma$. Hence, we may choose $\Gamma$ so that the center $Z=\{ \pm \mathrm{Id}\}$ of $\mathrm{SU}_{2}$ does not act on $M=T \rtimes \mathrm{SU}_{2}$ from the right. In other words, unlike in the example at the beginning of this section where it was possible to replace $M$ by its quotient with $Z$, there are no simple adjustments of $M$ which lead to a globalization.

One dramatic adjustment is to replace $M$ by its universal cover $\tilde{M}=\mathbb{C}^{N} \rtimes$ $\mathrm{SU}_{2}$ and then go down by the $Z$ quotient in $\mathrm{SU}_{2}$. This sort of isogeny does allow us to globalize, but unfortunately the compactness of $M$ is lost.

Note that we have the advantage in this construction that the $\mathrm{SL}_{2}(\mathbb{C})$ which is causing the trouble is a simple factor of $\widehat{G}$. We could have made matters worse by arranging an affine-rational fibration $\widehat{G} / \widehat{J} \rightarrow \widehat{G} / \widehat{Q}$ of the base of the anti-canonical bundle where $X$ now occurs as the fiber $\widehat{Q} / \widehat{J}$ and is an orbit of an $\mathrm{SL}_{2}(\mathbb{C})$ factor of the Levi-factor of $\widehat{Q}$.

The above example shows that whenever the affine quadric $\mathrm{SL}_{2}(\mathbb{C}) / D$ is involved in the fiber of the affine-rational fibration we must study the concrete case at hand in order to determine if $M$ is globalizable. If $N(D)$ is the normalizer of $D$, then its $2: 1$ quotient $\mathrm{SL}_{2}(\mathbb{C}) / N(D)$, which is the complement of a smooth conic in $\mathbb{P}_{2}(\mathbb{C})$, is equally dangerous. If $\widehat{G}$ is represented as a semisimple group on projective space and the affine-rational fibration has fiber $\widehat{Q} / \widehat{H} \cong \mathbb{C}$, then we can fiber further to $\widehat{G} / \widehat{H} \rightarrow \widehat{H} / \widehat{Q}_{1}$, where 
$\widehat{Q}_{1} / \widehat{Q} \cong \mathbb{P}_{1}(\mathbb{C})$ and $\widehat{Q}_{1} / \widehat{H}$ is again an affine quadric (AHR). So this case must also be handled on an individual basis. For simplicity of notation whenever any of these cases occur we say that the 2-dimensional affine quadric is involved.

Now the 2-dimensional affine quadric is the affine symmetric space which is the tangent bundle of the Hermitian symmetric space $S^{2}$. The phenomenon of nonglobalizability can be traced to our homotopy condition (see Proposition 3.1) not being satisfied. Recall that this states that if $G / J$ is a CR-manifold realized as an orbit in projective space with globalization $\widehat{G} / \widehat{J}$ there, then the inclusion $J \hookrightarrow \widehat{J}$ should induce a map $\pi_{1}(J) \rightarrow \pi_{1}(\widehat{J})$ with at most finite cokernel. The affine quadric is the only symmetric space of rank one where this does not occur: $J$ is trivial and $\widehat{J} \cong \mathbb{C}^{*}$. In the rank two case we find other examples where the homotopy condition is not satisfied. Although the affine quadric is a bit hidden in these examples, it is nevertheless involved. We now indicate how this happens.

Let $G$ be semisimple and compact, and let $M=G / J$ be an orbit in a projective space with globalization $\widehat{M}=\widehat{G} / \widehat{J}$ there. We suppose that $M$ is 2-codimensional in $\widehat{M}$. As we will see below, in order to understand the topological obstructions it is enough to handle the case where $\widehat{G} / \widehat{J}$ is affine and in that case it is enough to understand the situation where $\widehat{G} / \widehat{J}$ is the tangent bundle of a compact Hermitian symmetric $G / L$ space of rank two. There $G / L$ is a strong deformation retract of $\widehat{G} / \widehat{J}$. Thus our homotopy condition is reduced to showing that the natural map $\pi_{1}(J) \rightarrow \pi_{1}(L)$ has finite cokernel. To show that it is fulfilled it is therefore enough to show that the semisimple part $L_{s s}$ acts transitively on $L / J$.

One obvious case where $L_{s s}$ does not necessarily act transitively on $L / J$ is where $G / L$ is a reducible Hermitian symmetric space, i.e., a product of two Hermitian symmetric spaces of rank one. But $L_{s s}$ not acting transitively is equivalent to one of the factors being $S^{2}$; in particular the 2-dimensional affine quadric is involved.

We need a fact that can, for example, be read from Table $\mathrm{E}$ of ([GWZ) and, except for the symmetric space of $E_{6}$, can be directly verified by using the standard matrix models of the groups that occur. (For the list of Hermitian symmetric spaces see [Helg.).) If $G / L$ is an irreducible Hermitian symmetric 
space of rank two and $L_{s s}$ does not act transitively on the 2-codimensional generic orbit $L / J$ in the tangent space of the neutral point, then $G / L$ is one of the series of symmetric spaces $\mathrm{SO}_{p+2}(\mathbb{R}) / \mathrm{SO}_{p}(\mathbb{R}) \cdot \mathrm{SO}_{2}(\mathbb{R})$. The affine quadric is in fact involved in every such space from this series and by going to 2:1 coverings one constructs nonglobalizable examples. To show this we begin with the simplest example.

Let $G=\mathrm{SO}_{4}(\mathbb{R})=\left(\mathrm{SU}_{2} \times \mathrm{SU}_{2}\right) / \Gamma$, where $\Gamma$ is the diagonally embedded central subgroup of order two. Note that the diagonal subgroup $\mathrm{SU}_{2} / \Gamma$ can be identified with $\mathrm{SO}_{3}(\mathbb{R})$. In this case the Hermitian symmetric space is

$$
G / L=\mathrm{SO}_{4}(\mathbb{R}) / \mathrm{SO}_{2}(\mathbb{R}) \times \mathrm{SO}_{2}(\mathbb{R}),
$$

where the isotropy group is defined by the standard embedding of $\mathrm{SO}_{2}(\mathbb{R}) \times$ $\mathrm{SO}_{2}(\mathbb{R})$ in $\mathrm{SU}_{2} \times \mathrm{SU}_{2}$. The generic $G$-orbit $M:=G / J$ in $\widehat{G} / \widehat{J}$ is just $G$ itself, i.e., $J=\{e\}$. Now let $\mathrm{SO}_{3}(\mathbb{R})=\mathrm{SU}_{2} / \Gamma$ be embedded as above, define $x_{1}$ to be the base point in $G / L$ and $x_{0}$ to be the base point in $\widehat{G} / \widehat{J}$, where $M=G \cdot x_{0}=G / J=G$ and such that $x_{0}=g\left(x_{1}\right)$ with $g \in \mathrm{SO}_{3}(\mathbb{C})$. It follows that $M_{1}:=\mathrm{SO}_{3}(\mathbb{R})=\mathrm{SO}_{3}(\mathbb{R}) \cdot x_{0}$ is a CR-hypersurface in the affine quadric in $\mathrm{SO}_{3}(\mathbb{C}) / \mathrm{SO}_{2}(\mathbb{C})=\mathrm{SO}_{3}(\mathbb{C}) \cdot x_{1}$. Finally, let

$$
\widetilde{M}:=\mathrm{SU}_{2} \times \mathrm{SU}_{2}=\widetilde{G} \rightarrow G=M
$$

be the universal cover. Then $\mathrm{SU}_{2}=\widetilde{M}_{1}$ is the universal cover of $M_{1}$ equipped with its non-globalizable structure. Consequently, $\widetilde{M}$ is also not globalizable.

This low-dimensional example turns out to be a special case of a more general picture. For this, let us return to the affine-rational fibration $\widehat{G} / \widehat{J} \rightarrow \widehat{G} / \widehat{Q}$ of the globalization of the base of the $\mathfrak{g}$-anticanonical fibration. As was indicated above, it is enough to handle the case where the fiber $\widehat{Q} / \widehat{J}$ is an irreducible rank two affine symmetric space which is the tangent bundle of a Hermitian symmetric space. The above example fits as follows in this general context. Consider the Hermitian symmetric space $\mathrm{SO}_{p+2} / \mathrm{SO}_{p} \cdot \mathrm{SO}_{2}$. Then let $\mathrm{SO}_{p-2}(\mathbb{R}) \times \mathrm{SO}_{4}(\mathbb{R})$ be embedded in the usual block form so that the $\mathrm{SO}_{2}(\mathbb{R})$ of the isotropy group $\mathrm{SO}_{p}(\mathbb{R}) \cdot \mathrm{SO}_{2}(\mathbb{R})$ is diagonally embedded in $\mathrm{SO}_{4}(\mathbb{R})$ as above. As before, we let $x_{0}=g\left(x_{1}\right)$, where $x_{1}$ is a base point in the Hermitian symmetric space and $g \in \mathrm{SO}_{3}(\mathbb{C})$. It follows that $M=G \cdot x_{0}=$ $G / J$, where $J=\mathrm{SO}_{p-2}(\mathbb{R}) \times \Gamma$ with $\Gamma$ as above. Then analogous to that above, the universal cover $\widetilde{M}=G / J^{\circ}$ contains $\widetilde{M}_{1}=\mathrm{SU}_{2}$ which is equipped with a standard nonglobalizable structure. Thus $\widetilde{M}$ itself is nonglobalizable. 


\subsubsection{On the role of real projective space}

The case where $G$ is represented on the image of the anticanonical fibration as $\mathrm{SL}_{3}(\mathbb{R})$ with the image of $M$ being $G / J=\mathbb{P}_{2}(\mathbb{R})$ in its projective globalization $\mathbb{P}_{2}(\mathbb{C})$ is one further case where globalization might not be possible.

Similar to the above case, modulo ineffectivity, $\pi_{1}(\widehat{J})=\mathbb{Z}$ whereas $\pi_{1}(J)$ is trivial. In particular, our homotopy criterion does not guarantee globalization. On the other hand, in this case $\widehat{G} / \widehat{J}=\mathbb{P}_{2}(\mathbb{C})$ and so there is no mixing with fibrations. Furthermore, if we replace $G$ by the preimage of the maximal compact subgroup $\mathrm{SO}_{3}(\mathbb{R})$, then our homotopy condition does guarantee globalization, i.e., for the smaller group.

\subsubsection{Conditions for globalization}

Without going into a case-by-case study of exceptions, the following is the best globalization statement we presently know.

Theorem 5.7. If the affine quadric is not involved and the base G/J of the anticanonical fibration is not the real projective plane, then $M$ can be globalized. If $G$ is represented on the base of the $\mathfrak{g}$-anticanonical fibration as a compact group, then the only exceptions to the existence of globalization are where the affine quadric is involved.

Proof. We will apply the homotopy condition of Section 3. By Proposition 5.9 it is enough to handle the individual factors of the base of the anticanonical fibration. The exceptional case of the projective plane has been discussed above. The other case where a noncompact group is involved is where the factor of $G$ is represented as $\mathrm{SU}(p, q)$ or $\mathrm{Sp}(2 p, 2 q)$ and the projective $\mathrm{CR}$ homogeneous space of relevance is the mixed signature quadric. Here the homotopy condition of Section 3 is satisfied.

For example, to see this in the case of $\operatorname{Sp}(2 p, 2 q)$ we consider the decomposition $\mathbb{C}^{n}=V_{+} \oplus V_{-}$which is stabilized by a maximal compact subgroup $K \cong \operatorname{USp}(2 p) \times \mathrm{USp}(2 q)$. The $\mathrm{USp}(2 p)$-isotropy group of a nonzero vector $v_{+} \in V_{+}$acts on that vector by a nontrivial character. Since the same is true of $\operatorname{USp}(2 q)$ on $V_{-}$and we can choose $v=v_{+}+v_{-}$to be an isotropic vector, then a maximal compact subgroup of the $\operatorname{Sp}(2 p, 2 q)$-isotropy group at $v$ is just the $K$-isotropy $K_{v}$. The two characters mentioned above give us a representation of $K_{v}$ on $S^{1} \times S^{1}$ and the appropriately chosen diagonal will 
act by the same character on $v$ as does the $\mathrm{SL}_{n}(\mathbb{C})$-isotropy of the associated point in projective space. In particular, we see that the fundamental group of the isotropy group of the real form maps onto the fundamental group of the isotropy group of the complex form. This shows that the homotopy condition is satisfied in the case of $\operatorname{Sp}(2 p, 2 q)$. The case of $\mathrm{SU}(p, q)$ goes in a similar fashion.

Thus it remains to handle the case where $G$ is represented as a compact group on projective space and the affine quadric is not involved. For this we consider an affine-rational fibration $\widehat{G} / \widehat{J} \rightarrow \widehat{G} / \widehat{Q}$ of the globalization of the base of the anticanonical fibration. Since $G$ is compact, it acts transitively on the base. If the center $\widehat{Z}$ of $\widehat{Q}$ acts nontrivially on the fiber, we have an intermediate fibration

$$
\widehat{G} / \widehat{J} \rightarrow \widehat{G} / \widehat{Z} \widehat{J} \rightarrow \widehat{G} / \widehat{Q}
$$

The fiber $\widehat{Z} \widehat{J} / \widehat{J}$ is isomorphic to $\left(\mathbb{C}^{*}\right)^{n}, n=1,2$, and the induced fiber of the $G$-orbit is $\left(S^{1}\right)^{n}$. Thus the question of surjectivity of homotopy groups reduces to the same question after the intermediate fibration has been carried out. Hence, we may assume that the center of $\widehat{Q}$ acts trivially on the fiber.

In this case the fiber $\widehat{Q} / \widehat{J}$ is either $\mathbb{C}^{n}, n \geq 2$, or an affine spherical space under the action of a semisimple group. Since the affine quadric is not involved, this fiber is either an affine symmetric space of rank one or two or one of the two exceptional cases $\mathrm{SL}_{m+1} / \mathrm{SL}_{m}$ or $\mathrm{SO}_{9} / \mathrm{Spin}_{7}$. The proof is then completed by an analysis of homotopy groups which is carried out below.

We consider the long exact homotopy sequences of the fiber bundles in the rows of the following diagram.

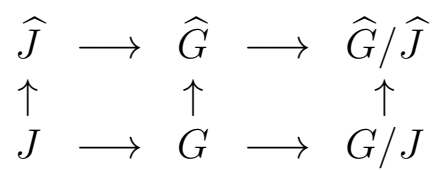

Analysis of these sequences shows that in order to guarantee the surjectivity of $\pi_{1}(J) \rightarrow \pi_{1}(\widehat{J})$ it is enough for

$$
\pi_{2}(G / J) \rightarrow \pi_{2}(\widehat{G} / \widehat{J})
$$

to be surjective. 
Now the minimal $G$-orbit $G / L$ in $\widehat{G} / \widehat{J}$ is a strong deformation retract of $\widehat{G} / \widehat{J}$. Thus we must show that the map $G / J \rightarrow G / L$ induces a surjective map at the level of $\pi_{2}$. Thus we look at the homotopy sequence of

$$
L / J \rightarrow G / J \rightarrow G / L .
$$

Since we are really only interested in surjectivity up to finite cokernel, we see that it is enough to show that $\pi_{1}(L / J)$ is finite. If $L$ is semisimple, this is immediate. So it remains to consider the case where $Q / L$ is a Hermitian symmetric space and $Q / J$ is a generic orbit (of codimension one or two) in its tangent bundle, i.e., $L / J$ is a generic orbit in its tangent space. As we showed above, unless the affine quadric is involved, $L_{s s}$ acts transitively on $L / J$ and thus $\pi_{1}(L / J)$ is finite. This then completes the proof of the Theorem.

\section{Fine Classification}

\subsection{The Levi-nondegenerate Case}

In this section suppose $M=G / H$ is a compact homogeneous CR-manifold of codimension at most two and assume that the Levi form of $M$ is nondegenerate. Let $G / H \rightarrow G / J$ be the $\mathfrak{g}$-anticanonical fibration of $M$. It is then possible to describe the fiber $J / H$ of the $\mathfrak{g}$-anticanonical fibration and to give a fine classification for $M$. The results in this setting in codimension one were given in $\mathrm{AHR}$.

We first note that by Proposition 2.1 the group $H^{\circ}$ is normal in $J=N_{C R}(H)$, since $N_{C R}(H) \subset N\left(H^{\circ}\right)$. Thus $N:=J / H^{\circ}$ is a group and $\Gamma:=J / H$ is a discrete subgroup of $N$. We also recall that there is a complexification $\widehat{N}$ of $N$; see Theorem 2.7. Without loss of generality one may assume that $\widehat{N}$ is simply connected and let $\widehat{N}=\widehat{R} \rtimes \widehat{S}_{\widehat{N}}$ be a Levi decomposition, where $\widehat{R}$ denotes the radical of $\widehat{N}$ and $\widehat{S}_{\widehat{N}}$ is a maximal semisimple subgroup of $\widehat{N}$. Let $\mathfrak{n}$ denote the Lie algebra of $N$ and $\mathfrak{m}_{\mathfrak{n}}$ be its (maximal) complex ideal. Further let $\widehat{\mathfrak{n}}$ denote the Lie algebra of the complexification $\widehat{N}$ of $N$ and assume $\widehat{\mathfrak{n}}=\widehat{\mathfrak{r}} \oplus \widehat{\mathfrak{s}_{\mathfrak{n}}}$ is a Levi decomposition of $\widehat{\mathfrak{n}}$, where $\widehat{\mathfrak{r}}$ denotes the radical of $\widehat{\mathfrak{n}}$ and $\widehat{\mathfrak{s}}_{\mathfrak{n}}$ a maximal semisimple subalgebra of $\widehat{\mathfrak{n}}$. In the proof of Theorem 2.7 we observed that

$$
\widehat{\mathfrak{s}}_{\widehat{\mathfrak{n}}} \subset \mathfrak{m}_{\mathfrak{n}} \subset \mathfrak{n}
$$

and we use this fact in the next result. 
Theorem 6.1. Suppose $M=G / H$ is a 2-codimensional compact homogeneous CR-manifold whose Levi form is nondegenerate. Let $G / H \rightarrow G / J$ be the $\mathfrak{g}$-anticanonical fibration of $M$ and let

$$
X=\widehat{G} / \widehat{H} \stackrel{\widehat{F}}{\longrightarrow} \widehat{G} / \widehat{J}
$$

be the corresponding globalization. Then the fiber $\widehat{F}$ is biholomorphic to $\left(\mathbb{C}^{*}\right)^{k}$ with $k \leq \operatorname{codim}_{X} M$ and $\widehat{N}$ is a complex Abelian Lie group of dimension at most two. Moreover,

- $\operatorname{dim}_{\mathbb{C}} \widehat{F}=0: M=G / H \rightarrow G / J$ is a (finite) covering and a maximal compact subgroup of $G$ acts transitively on $M$.

- $\operatorname{dim}_{\mathbb{C}} \widehat{F}=1: \widehat{F}=\mathbb{C}^{*}$, and the base $\Sigma:=G / J$ of the $\mathfrak{g}$-anticanonical fibration of $M$ is a compact, homogeneous $C R$-hypersurface that is equivariantly embedded in some projective space. In particular, $M$ fibers as an $S^{1}$-principal bundle over $\Sigma$.

- $\operatorname{dim}_{\mathbb{C}} \widehat{F}=2: \widehat{F}$ is, up to coverings, biholomorphic to $\mathbb{C}^{*} \times \mathbb{C}^{*}$, and the base $G / J=\widehat{G} / \widehat{J}=K / L=\widehat{K} / \widehat{P}$ of the $\mathfrak{g}$-anticanonical fibration of $M$ is a homogeneous rational manifold. One of the following occurs:

(i) $\widehat{P}$ acts transitively on $\widehat{F}$ and the complexification $\widehat{K}$ of $K$ acts transitively on $X$ yielding a $\widehat{K}$-equivariant fibration

$$
X=\widehat{K} / \widehat{I} \longrightarrow \widehat{K} / \widehat{P}
$$

that is a $\mathbb{C}^{*} \times \mathbb{C}^{*}$-principal bundle. Moreover, $M$ is an $S^{1} \times S^{1}$-principal bundle over the homogeneous rational manifold $G / J$.

(ii) $\widehat{L}$ is acting on $\widehat{F}$ as $\mathbb{C}^{*}$ and:

(a) $\widehat{P}$ is acting on $\widehat{F}$ as $\mathbb{C}^{*}$ and, up to finite coverings, $M$ admits a CR-splitting as a Levi non-degenerate hypersurface times $S^{1}$

(b) $\widehat{P}$ acts transitively on $\widehat{F}$ and there is a root $\mathrm{SL}_{2}$ associated to a particular root group $U$ in $\widehat{P}$. The parabolic group $\widehat{P}$ is a semidirect product $I_{\widehat{P}} \rtimes A$, where $I_{\widehat{P}}$ is the ineffectivity of the $\widehat{P}$-action on $\widehat{F}$ and $A$ is a 2-dimensional solvable group that is the semidirect product of a 1-dimensional torus with $U$. The isotropy $\widehat{I}$ of the $\widehat{K}$-action on $X$ is the subgroup $I_{\widehat{P}}$ extended by $U_{\mathbb{Z}}$, a subgroup of $U$ isomorphic to $\mathbb{Z}$. 
Proof. Since the Levi form of $M$ is nondegenerate, $\mathfrak{m}_{\mathfrak{n}}=(0)$. Thus by equation (6) one has $\widehat{\mathfrak{s}}_{\widehat{\mathfrak{n}}}=(0)$. So $N$ and $\widehat{N}$ are solvable.

If $\operatorname{dim}_{\mathbb{C}} \widehat{N}=0$, then one has a covering of one of the manifolds given by Theorem 5.6. Since the action of a maximal compact subgroup is transitive and algebraic on this projectively embedded manifold, the covering is finite. It is straightforward to determine what these are.

And, if $\operatorname{dim}_{\mathbb{C}} \widehat{N}=1$, the only possibility is $N / \Gamma=S^{1} \hookrightarrow \mathbb{C}^{*}=\widehat{N} / \Gamma$, because $\Gamma \subset N \subset \widehat{N}$. Then one has a $\mathbb{C}^{*}$-bundle over an $\widehat{S}$-orbit. If $\widehat{S}$ is not transitive on the total space of the bundle, then the $\widehat{S}$-orbits have codimension one in this total space and, up to finite coverings, they are sections. This implies that a finite covering is biholomorphic to a product. Otherwise, $\widehat{S}$ acts transitively on the total space of the bundle $X=\widehat{S} / \Gamma \rightarrow$ $\widehat{S} / \widehat{H}$ and its fiber is $\mathbb{C}^{*}$. Thus $M$ is given by an $S^{1}$-bundle over a compact hypersurface orbit of $S$ in $\widehat{S} / \widehat{H}$. The latter are described in detail in AHR and $\widehat{H}$ is acting on the fiber by a character. It is straightforward to work out the possibilities in this setting and we leave this to the reader.

So suppose $\operatorname{dim}_{\mathbb{C}} \widehat{N}>1$. By Proposition 7, pp. 50-51 in [HO] there exists a positive dimensional proper closed complex subgroup $\widehat{I}$ of $\widehat{N}$ that contains $\Gamma$ and without loss of generality one may assume that $\operatorname{dim}_{\mathbb{C}} \widehat{N} / \widehat{I}=1$. One also has the corresponding fibration $N / \Gamma \rightarrow N / I$, where $I:=N \cap \widehat{I}$ and $\widehat{N} / \Gamma \rightarrow \widehat{N} / \widehat{I}$. Now $\widehat{N} / \widehat{I}=\mathbb{C}, \mathbb{C}^{*}$, or a complex torus. The first case is not possible, since then by the codimension assumption, the fiber $I / \Gamma=\widehat{I} / \Gamma$ would be a compact complex manifold, contradicting the assumption that $M$ has a nondegenerate Levi form. Let's see that one cannot have a torus as base. Here we distinguish two cases depending on $\operatorname{codim}_{\mathbb{R}} M$. Suppose first that $M$ has a codimension two CR-structure. By induction the complex fiber $\widehat{N} / \widehat{I}$ is either $\mathbb{C}^{*} \times \mathbb{C}^{*}$ or the complex Klein bottle which is a two-to-one quotient of $\mathbb{C}^{*} \times \mathbb{C}^{*}$. Hence $\operatorname{dim}_{\mathbb{C}} \widehat{I}=2$ and thus $\operatorname{dim}_{\mathbb{C}} \widehat{N}=3$. Since $M=N / \Gamma$ fibers with real two dimensional fiber, $\operatorname{dim}_{\mathbb{R}} N=4$. Thus $\operatorname{dim}_{\mathbb{C}} \mathfrak{m}_{\mathfrak{n}}=1$, contradicting the assumption that the Levi form of $M$ is nondegenerate. So the base $\widehat{N} / \widehat{I}$ is biholomorphic to $\mathbb{C}^{*}$ and the fiber is $\mathbb{C}^{*}$, by induction. By the additivity of the codimensions of CR-structures, it follows that this is an $S^{1}$-bundle over $S^{1}$. In particular, $\operatorname{dim}_{\mathbb{C}} \widehat{N}=2$ and $\widehat{N} / \Gamma$ fibers as a $\mathbb{C}^{*}$-bundle over $\mathbb{C}^{*}$ which is either biholomorphic to a direct product or a two-to-one covering of it is biholomorphic to a product.

If the CR-structure of $N / \Gamma$ has codimension one, then $\widehat{I} / \Gamma$ is $\mathbb{C}^{*}$ by induction 
and if the base $\widehat{N} / \widehat{I}$ would be a one dimensional compact, complex torus, then $\operatorname{dim}_{\mathbb{C}} \widehat{N}=2$, while $\operatorname{dim}_{\mathbb{R}} N=3$. This again gives the contradiction that $\mathfrak{m}_{\mathfrak{n}} \neq(0)$. It follows that $\widehat{N} / \Gamma$ must be $\mathbb{C}^{*}$.

We will now determine the bundle structure in the case of a 2-dimensional fiber. Here we have the following setup at the level of globalizations:

$$
X=\widehat{G} / \widehat{H} \rightarrow \widehat{G} / \widehat{J},
$$

with fiber $\widehat{F}=\mathbb{C}^{*} \times \mathbb{C}^{*}$. The base $Z$ is of the form

$$
\widehat{S} / \widehat{Q}=S / I=\widehat{K} / \widehat{P}=K / L,
$$

where $K$ is a maximal compact subgroup of $S$ which is fixed for the discussion.

Our remarks revolve around the study of the actions of $\widehat{P}$ and $L$ on the fiber $\widehat{F}$. Since $\widehat{F}=\mathbb{C}^{*} \times \mathbb{C}^{*}$, any semisimple subgroup of $L$ acts trivially on $\widehat{F}$. Hence $L$ acts on $\widehat{F}$ as a solvable group and since $L$ is compact, $L$ is acting as an Abelian group. There are two cases that are relatively easy to handle.

First we assume that $L$ acts trivially on $F$. Then the ineffectivity of the $\widehat{P}$ action on $\widehat{F}$ is a normal subgroup of $\widehat{P}$ that contains a maximal torus. Since the unipotent radical of the parabolic group $\widehat{P}$ is a product of 1-dimensional subgroups and each of these is normalized by this maximal torus, it follows that this ineffectivity must be all of $\widehat{P}$. In other words, $\widehat{P}$ acts trivially on $\widehat{F}$. Thus the $\widehat{K}$-orbits which are the same as the $K$-orbits trivialize the bundle. However, this case does not occur because the Levi-form would be degenerate and we are assuming that this is not so. Next if $L$ acts transitively on $F$, then $\widehat{P}$ acts transitively on $\widehat{F}$ as an algebraic group. But then $\widehat{K}$ acts transitively on $X$ and $K$ acts transitively on $M$. This yields the principal bundle

$$
X=\widehat{K} / \widehat{I} \rightarrow \widehat{K} / \widehat{P}
$$

and the corresponding fibration of $M$ is an $S^{1} \times S^{1}$-principal bundle.

The remaining case occurs when the generic $L$-orbits are 1-dimensional. Throughout the discussion we will use the fact that $\hat{F}$ can be equivariantly fibered. There are two ways this can happen, depending on whether or not the full fiber group $\widehat{J}$ is acting as an Abelian group. In both cases, however, we have the intermediate fibration

$$
\widehat{G} / \widehat{H} \rightarrow \widehat{G} / \widehat{I} \rightarrow \widehat{G} / \widehat{J}
$$


where both fibers are $\mathbb{C}^{*}$. This induces an $S^{1}$-bundle fibration of the torus $F$ over $S^{1}$. If $L$ acts transitively on the base $\widehat{J} / \widehat{I}$ of this fibration, then it is clear that every $L$-orbit in $F$ is a copy of $S^{1}$ and that $L$ is acting as $S^{1}$. Otherwise, $L$ is only acting in the fibers of this fibration. But also in that case, linearizing the $L$-isotropy shows that $L$ acts transitively on all fibers and is acting as $S^{1}$. So in general $L$ can be regarded as acting by a free $S^{1}$-action and $L^{\mathbb{C}}$ by $\mathbb{C}^{*}$.

Again there are two cases:

1. The group $\widehat{P}$ is acting as $\mathbb{C}^{*}$.

2. The group $\widehat{P}$ acts transitively on $\widehat{F}$.

In the first case we claim that this only happens if $M$ is CR-isomorphic to the product of a Levi non-degenerate hypersurface with $S^{1}$. We look at the $\widehat{K}$-action on the base $\widehat{G} / \widehat{I}$ of the intermediate fibration in (77). If $\widehat{K}$ acts transitively on $\widehat{G} / \widehat{I}$, then (up to finite covers) the $\widehat{K}$-orbits in the total space $\widehat{G} / \widehat{H}$ are sections of the bundle $\widehat{G} / \widehat{H} \rightarrow \widehat{G} / \widehat{I}$. Let's go to such a covering. Then for $p \in M$, we consider $N=K \cdot p \hookrightarrow \widehat{K} \cdot p=Y$. The complex tangent space $T_{p}^{C R} M=T_{p}^{C R} N$ and is contained in the tangent space of the section $Y$. Now recall that the Levi-form is computed by bracketing $(1,0)$-fields with $(0,1)$-fields which are (complexified) tangent fields to $M$. In this case such fields will be tangent to $N$. Thus the values of the Levi-form lie in the tangent space to $Y$. Since we are assuming that the Levi-cone is open, then this is certainly not the case in the situation at hand, unless $M$ is a CR-product of a Levi non-degenerate hypersurface with $S^{1}$. Next assume that $\widehat{K}$ does not act transitively on the base. Hence the base $\widehat{G} / \widehat{I}$ is a product $\mathbb{C}^{*} \times Z$ and we go to its (canonically defined) holomorphic reduction. Since $L^{\mathbb{C}}$ is acting as $\mathbb{C}^{*}$, it follows that $\widehat{K}$ acts transitively on the fibers of the holomorphic reduction and thus we have an exact description of the situation - again, $M$ is the product of a Levi non-degenerate CR-hypersurface in the $\widehat{K}$-orbit with an $S^{1}$.

Finally, we come to the interesting case where $L^{\mathbb{C}}$ is acting as $\mathbb{C}^{*}$ and $\widehat{P}$ is acting transitively on the fiber $\widehat{F}$. Here we consider the $\widehat{P}$-action on the fiber of $\widehat{G} / \widehat{I} \rightarrow \widehat{G} / \widehat{J}$. This is a $\widehat{K}$-homogeneous $\mathbb{C}^{*}$-principal bundle where $\widehat{P}$ is acting by a character.

Since $L^{\mathbb{C}}$ is acting as $\mathbb{C}^{*}$ on $\widehat{F}$ it follows that the unipotent radical $R_{u}(\widehat{P})$ is acting with 1 -codimensional ineffectivity on the fiber $\widehat{J} / \widehat{H}$. Since this 
ineffectivity must also be a normal subgroup of $\widehat{P}$, it is a root group. Thus its complement in $\widehat{R}_{u}(\widehat{P})$ is an $L^{\mathbb{C}}$-invariant root group. Hence, we have a complete description of the $\widehat{P}$-ineffectivity on $\widehat{F}$ and it follows that $\widehat{P}$ splits as a semidirect product of this ineffectivity and a 2-dimensional solvable group $A$ which is a semidirect product of a 1-dimensional torus with a 1-dimensional simple root group $U$.

For the moment let us go to a covering of $X$ so that this ineffectivity is exactly the $\widehat{P}$-isotropy. In this way we reach a well-understood situation, because we can then fiber out the full reductive part of $\widehat{P}$ to obtain a $\mathbb{C}^{*}$-bundle over a $\mathbb{C}$-bundle where the simple root group acts transitively on the fiber. This $\mathbb{C}$ bundle setting, where $K$ has hypersurface orbits, has been classified in detail in $\mathrm{AHR}$. In particular, we find a further fibration $\widehat{K} / \widehat{P} \rightarrow \widehat{K} / \widehat{P}_{1}$ which is a $\mathbb{P}_{1}$-bundle. This is given by the root $\mathrm{SL}_{2}$ associated to the simple root group. Thus we see that the general example just comes from the standard 3-dimensional example $\mathrm{SL}_{2}(\mathbb{C}) / U_{\mathbb{Z}}$, where the $\mathrm{SL}_{2}(\mathbb{C})$ is embedded in the parabolic group $\widehat{P}_{1}$ as one of its semisimple factors. Here the isotropy in the bigger group $\widehat{K}$ is then the ineffectivity described above extended by a copy of $\mathbb{Z}$ in the simple root group at hand.

The basic example where $L$ acts on $F$ with 1 -dimensional orbits and $\widehat{P}$ acts transitively on $\widehat{F}$ arises as follows:

$$
X=\mathrm{SL}_{2}(\mathbb{C}) / U_{\mathbb{Z}} \rightarrow \mathrm{SL}_{2}(\mathbb{C}) / U=\mathbb{C}^{2} \backslash\{0\} \rightarrow \mathrm{SL}_{2}(\mathbb{C}) / B=\mathbb{P}_{1}(\mathbb{C}) .
$$

If we now consider the $\mathrm{SL}_{2}(\mathbb{C}) \times \mathbb{C}^{*}$-action on $X$ which is defined by the right $U$-action, then the real subgroup that has 2-codimensional orbits is $\mathrm{SU}_{2} \times S^{1}$. Note that $\mathrm{SO}_{3}(\mathbb{C})=\mathrm{SL}_{2}(\mathbb{C}) /( \pm \mathrm{Id})$ can be used to make essentially the same example. In that case we take $\widehat{K}$ to be the group which is acting effectively on the base (in this case $\mathbb{P}_{1}$ ) and therefore $L$ does indeed have 1-dimensional orbits.

This example can be put inside a bigger picture, i.e., the one that we describe in the classification proof. For this it is enough to consider the following example.

Example: Let $\widehat{G}=\mathrm{SL}_{n}(\mathbb{C})$ and $X=\widehat{G} / \widehat{P}$ be the Grassmannian of 2dimensional subspaces of $\mathbb{C}^{n}$. Let $\widehat{L} \ltimes R_{u}(\widehat{P})$ be the standard Levi decomposition of $\widehat{P}$, where $\widehat{L}=Z(\widehat{L})\left(\widehat{L}_{1} \times \widehat{L}_{2}\right)$ is the stabilizer of the decomposition

$$
\mathbb{C}^{n}=\operatorname{Span}\left\{e_{1}, e_{2}\right\} \oplus \operatorname{Span}\left\{e_{3}, \ldots e_{n}\right\}
$$


Here the center $Z(\widehat{L})$ acts diagonally. Now we attempt to build our basic example into $\widehat{P}$. Since $\widehat{L}_{1}=\mathrm{SL}_{2}(\mathbb{C})$, we may define $\widehat{H}=Z(\widehat{L})\left(U_{\mathbb{Z}}\left(\widehat{L}_{1}\right) \times \widehat{L}_{2}\right)$. Since $U\left(\widehat{L}_{1}\right)$ normalizes $\widehat{H}$, we have the induced right-action of $U / U_{\mathbb{Z}}$ and, redefining our group as the larger group $\widehat{G} \times U / U_{\mathbb{Z}}$, we have the desired example.

Remark: It should be noted that in general the semisimple factor $\widehat{L}_{1}$ may be $\mathrm{SO}_{3}(\mathbb{C})$ and therefore we must build in the second basic example mentioned above. Furthermore, to put this in the context of our classification result in the setting where $L$ acts as $S^{1}$ on the fiber, we must choose $\widehat{K}$ to be the group which is acting effectively on the base $Z$.

\subsection{The Kählerian Parallelizable Case}

As we have seen, the only remaining serious difficulty in determining a fine classification in the general 2-codimensional case is when the fiber of the $\mathfrak{g}$ anticanonical bundle is also 2-codimensional, i.e., when the base is a compact complex manifold. In this case we must understand the fiber in its globalization, and therefore it is enough to consider the case where the base is just a point. Thus in this section we assume that $M=G / \Gamma$, where $\Gamma$ is discrete.

By the considerations in $\S 2.3$ one knows that there exists a complexification $(X, \sigma)$ of $G / \Gamma$, where we think of $M$ as already embedded in $X$, i.e., we identify $M$ with $\sigma(G / \Gamma)$. In this section we assume that $M$ is Kählerian in the sense that $M$ is contained in a tube $Z$ in $X$ that is Kähler. We also recall that the Lie algebra $\mathfrak{g}$ of $G$ can be complexified to a Lie algebra $\widehat{\mathfrak{g}}$ that acts locally on some open neighborhood of $M$ in $Z$, see $\S 2.4$. Note that without loss of generality we may assume that this neighborhood is $Z$ itself by appropriately shrinking and renaming, if necessary. As usual we let $\mathfrak{m}:=\mathfrak{g} \cap i \mathfrak{g}$ be the maximal complex ideal in $\mathfrak{g}$. The purpose of this section is to show that $\mathfrak{m}$ is solvable in this general Kähler case. In addition, if $M$ has a CR-structure of codimension at most two, then $\mathfrak{g}$ is solvable.

Theorem 6.2. Let $\Gamma$ be a discrete cocompact subgroup of $G$ and assume that the compact $C R$-homogeneous space $M:=G / \Gamma$ is Kählerian in the sense considered above. Then the maximal complex ideal $\mathfrak{m}$ in $\mathfrak{g}$ is solvable. In particular, if the CR-structure of $M$ is at most 2-codimensional, then $G$ is solvable. 
For the proof we apply several of the basic tools for the theory of quotients via Kählerian reduction (see $[\mathrm{HH}]$ ). Here we give a streamlined argument using the strong assumptions at hand. For this let $L$ be the complex semisimple Lie subgroup of $G$ which is associated to a maximal semisimple subalgebra $\mathfrak{l}$ of $\mathfrak{m}$ and let $K$ be a fixed choice of a maximal compact subgroup of $L$. Note that we may assume that the tube $Z$ is $K$-invariant. By averaging the given Kähler form $\omega$ over $K$ we may assume that it is $K$-invariant. Thus, since $K$ is semisimple, the general theory guarantees the existence of a unique $(K-$ equivariant) moment map $\mu: Z \rightarrow \mathfrak{k}^{*}$. Finally, define $M_{0}:=\mu^{-1}(0) \cap M$. Note that since the $K$-action on $Z$ is locally free, $\mu$ is an open mapping of constant rank which is equal to $\operatorname{dim}_{\mathbb{R}} K$.

Proposition 6.1. The 0-fiber $\mu^{-1}(0)$ is a submanifold of $Z$ which is transversal to L.x at every point $x \in M_{0}$. In particular, $M_{0}$ is smooth, nonempty and $M^{s s}:=L \cdot M_{0}$ is open in $M$.

Proof. The moment map associated to the pullback of $\omega$ to the orbit L.x of a point $x \in M_{0}$ is just the pullback of the moment map. Since the $K$-action is locally free, the ranks of these two maps are the same and the transversality statement is immediate. Hence, it only remains to show that $M_{0}$ is nonempty. But, since $\mu \mid L . x$ is an open map for all $x \in M$, if $x_{0}$ is a point of $M$ where $\|\mu\|^{2} \mid M$ takes on its minimum, it follows that $x_{0} \in M_{0}$.

By definition, the complement $M_{1}:=M \backslash M^{s s}$ is a compact $L$-invariant subset of $M$. Just as in the above argument, if $M_{1}$ were nonempty and $\|\mu\|^{2} \mid M_{1}$ takes on its minimum at $x_{1}$, then $x_{1}$ would be in $M_{0}$, contrary to assumption.

\section{Corollary 6.1.}

$$
M^{s s}=L \cdot M_{0}=M .
$$

Now, for $x \in M_{0}$ the orbit L.x is retractible to the $K$-orbit $K . x$ which is isotropic in L.x. Since L.x is Stein, it then follows that $\omega=d d^{c} \rho$ on $L . x$, where $\rho$ is a $K$-invariant strictly plurisubharmonic function. By the results of Azad and Loeb ( $(\mathrm{AL}])$, if $m_{0}:=\rho\left(x_{0}\right)$, then $\rho: L_{x} \rightarrow\left[m_{0}, \infty\right)$ is a proper exhaustion with no critical points except for those where it takes on its minimum along $K . x_{0}$. In particular, L.x $\cap M_{0}=K . x$ for all $x \in M_{0}$.

Proof of Theorem 6.2. Since for every $x \in M$ the orbit L.x is Kähler and the isotropy group $L_{x}$ is just the intersection with $L$ of some conjugate of $\Gamma$ in 
$G$, it follows that $L_{x}$ is a discrete subgroup of $L$. The fact that $L . x$ is Kähler then implies that $L_{x}$ is finite $([\mathrm{BeOe}])$. By replacing $\Gamma$ by an appropriate subgroup of finite index, we may assume that $\Gamma$ is torsion free and therefore that the $L$-action on $M$ is free. Since $L \cdot M_{0}=M$, it therefore follows that the action map $L \times M_{0} \rightarrow M,(\ell, x) \rightarrow \ell(x)$, identifies $M$ with the noncompact bundle space $L \times_{K} M_{0}$. Unless $L$ is trivial, this is a contradiction. Thus $\mathfrak{m}$ is solvable.

Finally, if the CR-structure of $M$ is at most 2-codimensional, then $\mathfrak{m}$ is also at most 2-codimensional in $\mathfrak{g}$ and consequently $\mathfrak{g}$ is also solvable.

Remark. In the hypersurface case finer results are known [Ri]. At present we only know rather simple constructions of examples using solvable (nonabelian) groups in the 2-codimensional case, e.g., complexifications of compact 2-dimensional solv-manifolds. As a Leitfaden for more interesting constructions, it might be of interest to attempt to build examples $X$ with $\mathcal{O}(X)=\mathbb{C}$.

\section{References}

[Ak1] D.N. Akhiezer, Actions with a finite number of orbits, Funct. Anal. Appl. 19 (1985), 1-4.

[Ak2] D.N. Akhiezer, Spherical Varieties, Schriftenreihe, Heft Nr. 199, 1993, Ruhr-Universität-Bochum, 52 pp.

[AF] A. Andreotti, G.A. Fredrichs, Embeddability of real analytic Cauchy-Riemann Manifolds, Ann. Scuola Norm. Pisa 6 (1979), 285 304.

[AHR] H. Azad, A. Huckleberry, W. Richthofer, Homogeneous $C R$ Manifolds. J. Reine Angew. Math. 358 (1985), 125-154.

[AL] Azad, H. And Loeb, J.-J., Plurisubharmonic functions and the Kempf-Ness Theorem. Bull. London Math. Soc. 25 (1993), 162 - 168.

[AS] D. Alekseevskky and A. Spiro, Compact homogeneous CRmanifolds, J. Geom. Anal. 12 (2002), 183 - 201. 
[BeOe] F. Berteloot and K. Oeljeklaus, Invariant plurisubharmonic functions and hypersurfaces on semi-simple complex Lie groups. Math. Ann. 281 (1988), 513 - 530.

[Bor] A. Borel, Linear algebraic groups. W. A. Benjamin, Inc., New YorkAmsterdam 1969 xi+398 pp.; Second Edition: Springer-Verlag, New York, 1991.

[BR] Borel, A.: Remmert, R., Über kompakte homogene Kählersche Mannigfaltigkeiten, Math. Ann. 145 (1962), 429 - 439.

[Bri] M. Brion, Spherical varieties: an introduction. Topological methods in algebraic transformation groups (New Brunswick, NJ, 1988), 11-26, Progr. Math., 80, Birkhuser Boston, Boston, MA, 1989.

[BLV] M. Brion, D. Luna, Th. Vust, Espaces homogènes sphériques, Invent. Math. 84 (1986), 617-632.

[Chev] C. Chevalley, On the topological structure of solvable groups. Ann. of Math. (2) 42, (1941). 668-675.

[FHW] G. Fels, A. Huckleberry, J.A. Wolf, Cycle spaces of flag domains. A complex geometric viewpoint. Progress in Mathematics, 245. Birkhuser Boston, Inc., Boston, MA, 2006. xx+339 pp.

[FK] G. Fels and W. Kaup, CR-manifolds of dimension 5: a Lie algebra approach, J. Reine Angew. Math. 604 (2007), 47 - 71.

[Goto] Goto, N., Faithful representations of Lie groups. I., Math. Japonicae, 1 (1948), 107 - 119.

[Gr] Greenfield, S., Cauchy-Riemann equations in several variables. Ann. Scuola Norm. Pisa, 22 (1968), 257 - 314.

[GWZ] Grove, K., Wilking, B., Ziller, W., Positively curved cohomogeneity one manifolds and 3-Sasakian geometry arxiv:math.DG/0511464.

[GS] Guillemin, V., Sternberg, S., Symplectic techniques in Physics, Cambridge: Cambridge Univ. Press, 1984. 
[Helg] S. Helgason, Differential Geometry and Symmetric Spaces. Pure and Applied Mathematics, 80. Academic Press, Inc. [Harcourt Brace Jovanovich, Publishers], New York-London, 1978. xv+628 pp.

[HH] Heinzner, P. and Huckleberry, A., Complex geometry of Hamiltonian actions, Springer-Verlag (to appear 2009).

[HO] A. Huckleberry and E. Oeljeklaus., Classification theorems for almost homogeneous spaces. Institut lie Cartan, 9. Universit de Nancy, Institut lie Cartan, Nancy, 1984. iv+178+xiv pp.

[HR] A. Huckleberry and W. Richthofer, Recent developments in homogeneous CR-hypersurfaces. Contributions to several complex variables, 149-177, Aspects Math., E9, Vieweg, Braunschweig, 1986.

[HW] A. Huckleberry and T. Wurzbacher, Multiplicity-free complex manifolds. Math. Ann. 286 (1990), no. 1-3, 261-280.

[Hum] James E. Humphreys, Linear algebraic groups. Graduate Texts in Mathematics, No. 21., Springer-Verlag, New York-Heidelberg, 1975. xiv +247 pp.

[I] A. Isaev, Lectures on the Automorphism Groups of KobayashiHyperbolic Manifolds, LMN 1902, Springer-Verlag (2006)

[IK] A. Isaev and N. Kruzhilin, Effective actions of $\mathrm{SU}_{n}$ on complex n-dimensional manifolds, Illinois J. Math., 48, 37-57 (2004)

[KZ] W. Kaup And D. Zaitsev, On local CR-transformation of Levidegenerate group orbits in compact Hermitian symmetric spaces. J. Eur. Math. Soc. (JEMS) 8 (2006), no. 3, 465-490.

[KN2] Kobayashi and Nomizu, Foundations of Differential Geometry, Volume II, Interscience Publishers, New York, London, 1963.

[Kr] KrÄmer, M., Sphärische Untergruppen in kompakten zusammenhängenden Liegruppen, Compositio Math. 38 (1979), 129-153.

[L] A. Loboda, Locally homogeneous strongly pseudoconvex CRmanifolds, Sbornik Mathmatics, 192 (2001) 1741-1761. 
[LV] Luna, D. And Vust, Th., Plongements d'espaces homogénes. Comment. Math. Helv. 58 (1983), 186-245.

[Mat1] T. MATsuki, Orbits on affine symmetric spaces under the action of parabolic subgroups, Hiroshima Math. J. 12 (1982), 307-320.

[Mat2] T. Matsuki, Double coset decompositions of reductive Lie groups arising from two involutions, J. of Alg. 197 (1997), 49-91.

[Mont] Montgomery, D., Simply connected homogeneous spaces. Proc. Amer. Math. Soc. 1, (1950). 467-469.

[MN] Morimoto, A. And Nagano, T., On pseudo-conformal transformations of hypersurfaces. J. Math. Soc. Japan 15 (1963), 289-300.

[N] T. Nagano, Transformation groups with $(n-1)$-dimensional orbits on non-compact manifolds. Nagoya Math. J. 141959 25-38.

[O1] A. L. Onishchik, Inclusion relations among transitive compact transformation groups. Trudy Moskov. Mat. Obšč. 11 (1962), 199-142.

[O2] A. L. Onishchik, Topology of Transitive Transformation Groups, Johann Ambrosius Barth, Leipzig/Berlin/Heidelberg, 1994.

[Ri] Richthofer, W., Homogene $C R$-Mannigfaltigkeiten, Ph.D. Dissertation, Ruhr-Universität Bochum, 1985.

[Ro] H. Rossi, Homogeneous strongly pseudoconvex hypersurfaces, Rice Univ. Studies, 5 No.1 (1973), 131-145

[VK] VinberG, E.S. And Kimelfeld, B.N., Homogeneous domains on flag manifolds and spherical subsets of semisimple Lie groups, Funct. Anal. Appl. 12 (1979), 168-174.

Authors' addresses:

Dept. of Math. \& Stats.

Fakultät und Institut für Mathematik

University of Regina

Ruhr-Universität Bochum

Regina, Canada S4S 4A5

D-44780 Bochum, Germany

gilligan@math.uregina.ca

ahuck@cplx.ruhr-uni-bochum.de 